\title{
Identifying Software Defects Using Neural Graph Classifiers
}

\author{
By \\ Olivier Hamel, B.Cs.

\begin{abstract}
A thesis submitted to the
Faculty of Graduate and Postdoctoral Affairs

in partial fulfilment of the requirements for the degree of
\end{abstract}

Master in Computer Science

Ottawa-Carleton Institute for Computer Science

The School of Computer Science

Carleton University

Ottawa, Ontario

(C) Copyright

Olivier Hamel, 2018 


\section{Abstract}

Static analysis tools are commonly used to assist with program verification. Most existing tools, however, identify defects using a predefined repertoire of defect specifications. These repertoires are rarely extended by users due to the effort and complexity involved in producing new specifications.

I propose a supervised approach for learning defective code patterns directly from coarsely labelled examples, allowing users to produce new defect classifiers with minimal effort. This approach represents a program as a directed multi-graph modelling operations and their dependencies, and aims to identify defects caused by incorrect, or missing, dependencies between operations. Given this representation, the task of identifying these defects can be seen as identifying specific partial subgraphs.

The proposed approach is evaluated against several open source projects and test suites targeting the Java virtual machine (JVM). The proposed approach performs well against an adapted subset of the Juliet [60] test suite, and demonstrates a modest ability to transfer to unseen natural code. 


\section{Acknowledgements}

I wish to thank Dr. Guo, and everyone else whom I pestered for feedback and review, for their infinite patience and support.

For my mother, Ann. 


\section{Table of Contents}

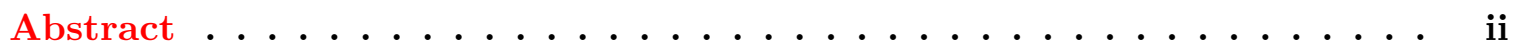

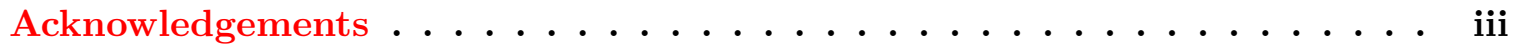

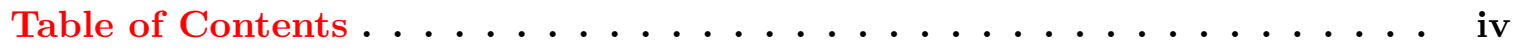

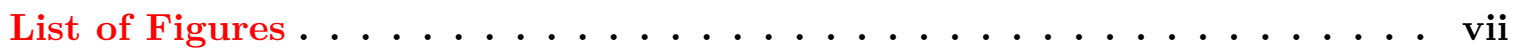

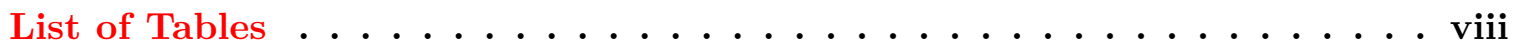

Listings ......................... ix

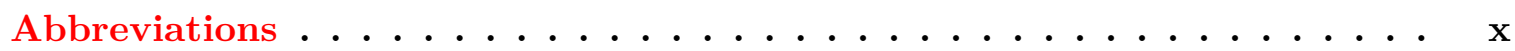

1 Introduction ......................... 1

1.1 Problem Statement . . . . . . . . . . . . . . . . . . . 1

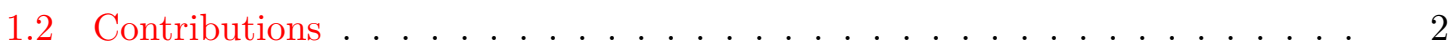

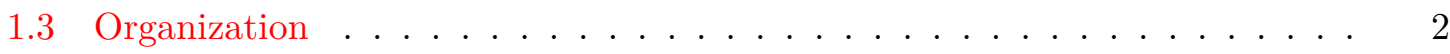

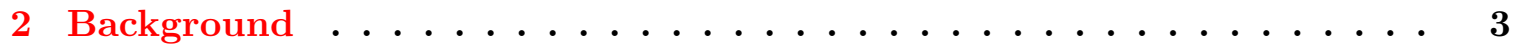

2.1 Program Representations . . . . . . . . . . . . . . . . . 3

2.1.1 Control \& Data Flow Graphs . . . . . . . . . . . . . 5

2.1 .2 Single Static Assignment . . . . . . . . . . . . . . . . 6

2.2 Type Systems . . . . . . . . . . . . . . . . . . . . . 6

$2.2 .1 \quad$ JVM Type System . . . . . . . . . . . . . . . . . 7

2.3 Neural Networks . . . . . . . . . . . . . . . . . . . . 8

2.3.1 Activation Functions . . . . . . . . . . . . . . . 9

$2.3 .2 \quad$ Feed-forward Networks . . . . . . . . . . . . . . . . . . . . . 10

2.3.3 Recurrent Networks . . . . . . . . . . . . . . . . . . . . 11

2.3.4 Graph Networks . . . . . . . . . . . . . . . 11

2.4 Structured Representations . . . . . . . . . . . . . . . . . 14 
3 Related Work ........................ 16

3.1 Program Representations . . . . . . . . . . . . . . . . . . 16

3.2 Supervised Classification of Programs . . . . . . . . . . . . . . . 19

3.3 Outlier-based Defect Detection . . . . . . . . . . . . . . . 20

3.4 Specification Inference . . . . . . . . . . . . . . . . . . . 21

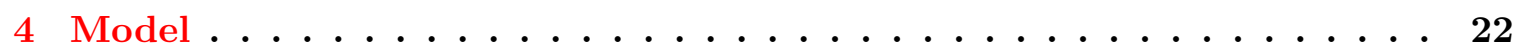

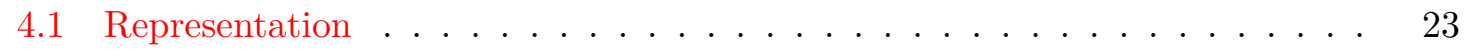

4.1 .1 Dependence Graphs . . . . . . . . . . . . . . . . . 24

4.1 .2 Graph Simplification . . . . . . . . . . . . . . 24

4.1 .3 Vertex \& Edge Features . . . . . . . . . . . . . . . . . . . 27

4.2 Neighbourhood Embedding . . . . . . . . . . . . . . . . 32

4.3 Objective Function . . . . . . . . . . . . . . 34

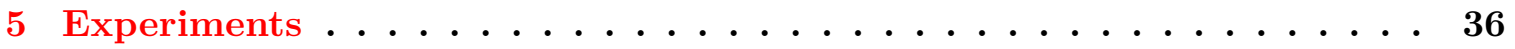

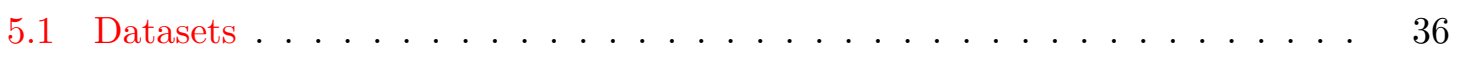

5.1 .1 Synthetic Projects . . . . . . . . . . . . . . 37

5.1 .2 Natural-Negative Projects . . . . . . . . . . . . . . . . . . 39

5.1 .3 Natural-Defective Projects . . . . . . . . . . . . . . . 41

5.1 .4 Dataset Extraction \& Handling . . . . . . . . . . . . . . . . . 42

5.2 Training Protocol . . . . . . . . . . . . . . . . . . . . 45

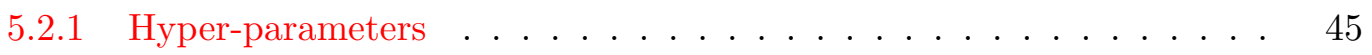

5.3 Primary Results . . . . . . . . . . . . . . . . . . 45

5.3 .1 Feature-set Ablation . . . . . . . . . . . . . 47

5.4 Transferring to Unseen Projects . . . . . . . . . . . . . . . . 51

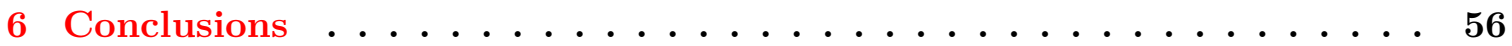

6.1 Future Work . . . . . . . . . . . . . . . . 56

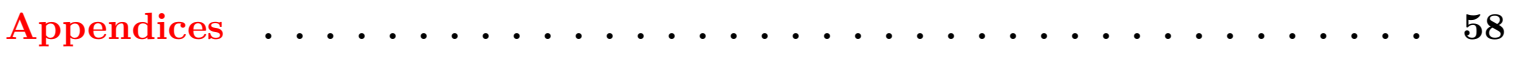

A Recurrence Connection Behaviour ............... 58

B Natural-Negative Dataset Projects ............... 62 


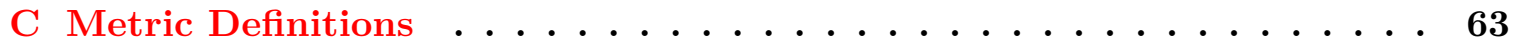

D Feature-set Ablation Results — All Samples ............ 65

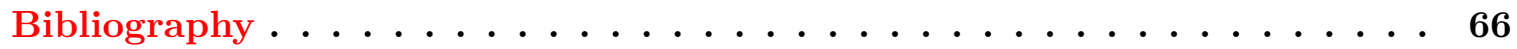

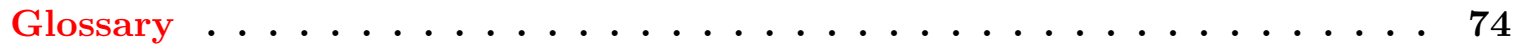




\section{List of Figures}

2.1 Typical compiler pipeline and representations . . . . . . . . . . . 4

2.2 Data-flow graph example . . . . . . . . . . . . . 6

2.3 SSA example . . . . . . . . . . . . . . . . . 7

2.4 Common neural network activation functions . . . . . . . . . . . . . 10

2.5 Illustration of message passing f . . . . . . . . . . . . . . . 12

4.1 Model architecture overview . . . . . . . . . . . . . . . . . 23

4.2 Source code and corresponding dependence graph . . . . . . . . . . . 26

4.3 Examples of RHS and $\phi$-dependence simplification. . . . . . . . . . 28

5.1 Performance metric curves for primary dataset . . . . . . . . . . . . 49

5.2 Performance metric curves for Juliet only . . . . . . . . . . . . . . . . 51

5.3 Performance metric curves for Natural-Defective dataset . . . . . . . . . . . 54

A.1 Null-Input h Steady States . . . . . . . . . . . . . . . . 61 


\section{List of Tables}

2.1 Graph neural network variants . . . . . . . . . . . . . . 13

4.1 Soot optimization passes . . . . . . . . . . . . . . 25

4.2 List of operation and dependence features. . . . . . . . . . . . . 28

4.3 String representations for numeric constant values . . . . . . . . . . . 31

4.4 List of dependence types. . . . . . . . . . . . . . . . . . . . 32

5.1 Dataset source categories . . . . . . . . . . . . . . . . 37

5.2 Juliet dataset population . . . . . . . . . . . . . . . . 38

5.3 Subgraph radii for the connected components within the Juliet dataset. . . 38

5.4 Natural-defective dataset population . . . . . . . . . . . . . . . . 41

5.5 Parameter initializers . . . . . . . . . . . . . . . . . . 45

5.6 Hyper-parameters . . . . . . . . . . . . . . . . 46

5.7 Results for Juliet + Natural-Negative _ . . . . . . . . . . . . . . . 48

5.8 Juliet performance per (CWE, variant) for $k=10$ model . . . . . . . . . 50

5.9 Feature-set ablation evaluation for primary dataset, using baseline and $k=5$ models . . . . . . . . . . . . . . . . . . 52

5.10 Evaluation on natural-defective . . . . . . . . . . . 55

B.1 Natural-negative dataset population . . . . . . . . . . . . . 62

D.1 Full feature-set ablation evaluation for primary dataset, using baseline and $k=5$ models . . . . . . . . . . . . . . . . . 65 


\section{Listings}

5.1 Reduced example of a Juliet test case. . . . . . . . . . . . . . . . 40

5.2 Overfitted example using common symbol in unrestricted symbol vocabulary. 44

5.3 Overfitted example using rare symbol in unrestricted symbol vocabulary. . . 44 


\section{Abbreviations}

\begin{tabular}{|c|c|}
\hline $\mathrm{AP}$ & average precision \\
\hline API & application programming interface \\
\hline $\mathrm{AST}$ & abstract syntax tree \\
\hline $\mathrm{AUC}$ & area under curve \\
\hline BPTT & backpropagation through time \\
\hline CBOW & continuous bag of words \\
\hline $\mathrm{CFG}$ & control-flow graph \\
\hline CFR & chaos-free recurrence \\
\hline CWE & Common Weakness Enumeration \\
\hline DFG & data-flow graph \\
\hline DSL & domain specific language \\
\hline FC LAYER & fully-connected layer \\
\hline FDR & false discovery rate \\
\hline FPR & false positive rate \\
\hline GRU & Gated Recurrent Unit \\
\hline IPA & interprocedural analysis \\
\hline IR & intermediate representation \\
\hline JVM & Java virtual machine \\
\hline LSTM & Long Short-Term Memory \\
\hline MLP & multi-layer perceptron \\
\hline $\mathrm{OOV}$ & out-of-vocabulary \\
\hline RHS & right-hand-side expression \\
\hline RNN & recurrent neural network \\
\hline $\mathrm{ROC}$ & receiver operating characteristics \\
\hline SGD & stochastic gradient descent \\
\hline SQL & Structured Query Language \\
\hline SRNN & simple recurrent neural network \\
\hline SSA & single static assignment \\
\hline
\end{tabular}


UTF-8 Unicode Text Format 8-bits 


\section{Chapter 1}

\section{Introduction}

Static analysis tools are frequently used to assist with the verification of software. However, their capabilities are limited by the fact that almost all programs lack a complete machineverifiable specification against which they can be verified. This is unfortunate since the defectiveness of a behaviour can depend on the program's functional requirements. As a result, most static analysis tools verify programs against specifications derived from the programming language's own specification, assuming one exists, or a set of ad-hoc specifications covering common application-agnostic defects. This set of specifications constitutes an analyzer's repertoire of known defects.

While some tools support customization of their repertoire of specifications, adding or refining specifications often requires specialist knowledge orthogonal to that required to identify and characterize the defect under consideration. This dissuades users from customizing a specifications reporting false positives for their program, or adding new specifications to cover a false negative.

\subsection{Problem Statement}

The objective of this work is to explore the feasibility of applying machine learning techniques to learn defect detectors from examples. Users should be able to label examples by simply specifying the lines of code which induce the defect, while the classifiers produced from this data should be capable of issuing predictions with sub-function level granularity.

This per-line approach is intended to reduce the effort required by a user to label a positive sample. In exchange, we accept that the labels provided will be coarse; they may only partially intersect with the true set of operations which induce the defect in question. 


\subsection{Contributions}

I propose a new supervised approach for identifying application defects caused by incorrect, or missing, dependencies between operations. This is accomplished by learning to identify partial subgraphs within a graphical representation of a program's operation dependencies using learned inductive representations for each operation. These learned representations are produced using a graph neural network. By specifically considering dependencies between operations (such as subroutine invocations), the proposed approach is well suited for identifying defects caused by improper application programming interfaces (APIs) usage.

I demonstrate that this approach is effective against an adapted subset of the Juliet test suite [60], and that it can weakly transfer to unseen natural code.

\subsection{Organization}

We begin with a brief review of relevant background material in chapter 2, covering program representations and common neural models. Chapter 3 discusses related works for graph embedding, graphical neural models, and program modelling. The proposed approach is presented in chapter 4 , and experimental results are presented in chapter 5 , including ablative studies. Chapter 6 presents my conclusions, and outlines avenues for future work. 


\section{CHAPTER 2}

\section{Background}

This chapter provides background information on neural network models, program representations, and a brief discussion on handling structured inputs. The intent of this chapter is to establish a basic context for this work, as well as works discussed in chapter 3 .

\subsection{Program Representations}

A program can be expressed using different representations, each specialized for specific use cases. For example, programs are typically written in a human-friendly notation (source code) and then mechanically translated into a form interpretable by a computer (machine code). A typical pipeline for this process is depicted in fig. 2.1. These two representations are quite dissimilar: the former is a sequence of characters, while the latter is a sequence of data and instructions. The translation from one to the other requires significant prior knowledge regarding both representations.

This prior knowledge requirement makes it difficult to reliably infer program properties from the source code or compiled form. However, a well-defined program ${ }^{1}$ has precise semantics which can be mechanically evaluated. Instead of inferring the syntax or low level semantics from source code, one can obtain them directly using a compiler's machinery and representations. These representations can be classified as either syntactic or semantic.

Syntactic Representations. Syntactic representations are used by compilers to verify the encoding of unstructured source code text and translate it into a structured form. This is typically accomplished in two phases:

\section{Lexical analysis}

\footnotetext{
${ }^{1}$ For some language-defined notion of 'well-defined.'
} 


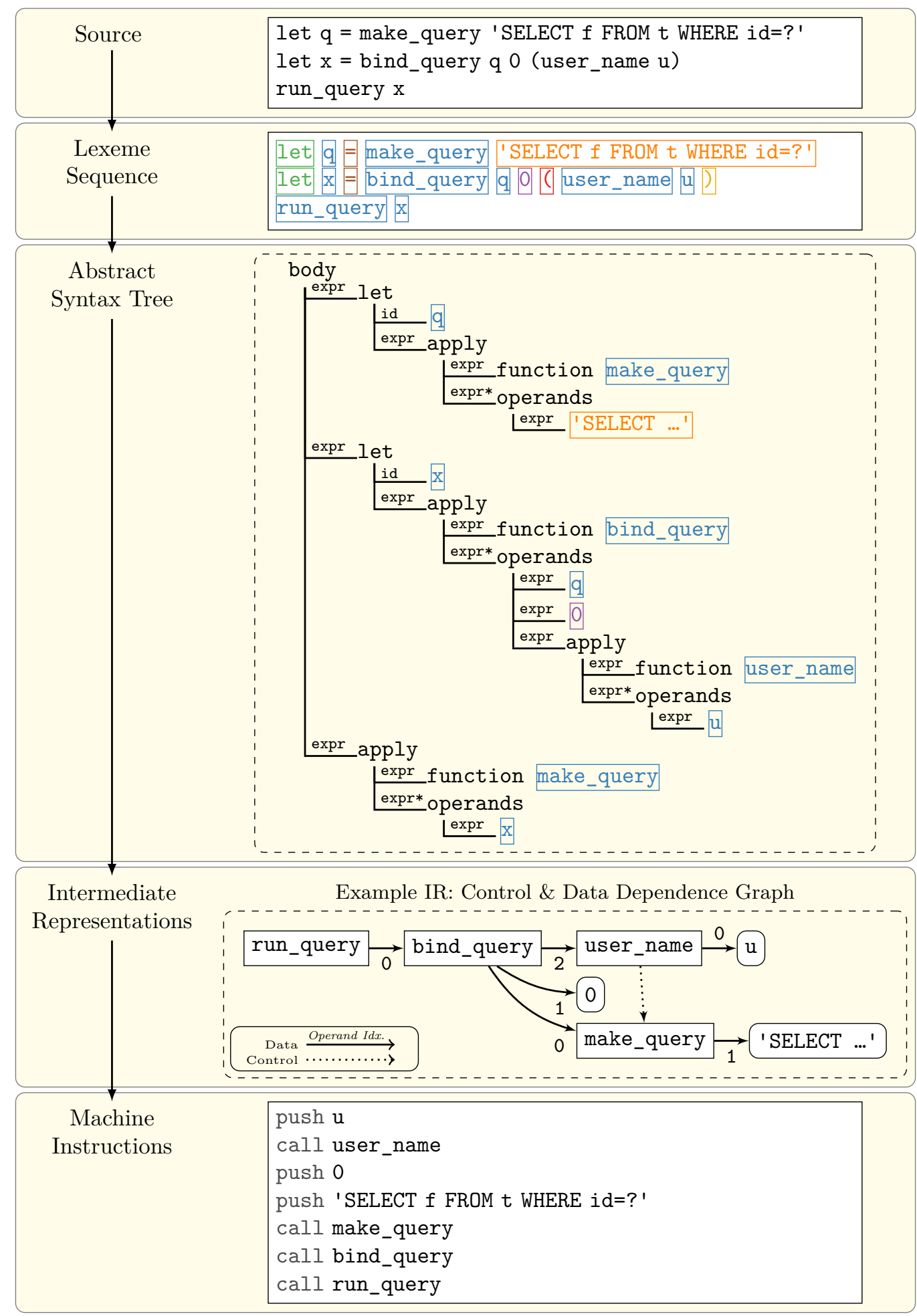

Fig. 2.1: A typical compiler pipeline for translating source code into executable code. In this example, a toy language is compiled to a stack machine. The majority of a compiler's complexity lies within the intermediate representations (IRs), where semantic analyses and transformations occur. 


\section{Grammatical analysis}

Lexical analysis (lexing) transforms the source code from a sequence of characters into a sequence of lexemes. Grammatical analysis (parsing) transforms the sequence of lexemes into an abstract syntax tree (AST) for the language in question.

Semantic Representations. Semantic representations, or IRs, are data structures used to reason about a program's meaning, prove properties, and justify various transformations and optimizations. It is common to use multiple IRs, each modelling some specific aspect of the program, and most can be naturally expressed as directed graphs. Three particularly important IRs, control-flow graphs (CFGs), data-flow graphs (DFGs), and single static assignment (SSA), are introduced below.

\subsubsection{Control \& Data Flow Graphs}

Practically all program analyses depend upon reasoning about possible paths of execution through a program, and how values are computed or consumed. This information can be obtained through various control flow analyses (CFA) and data flow analyses (DFA), the results of which are directed graphs describing the control or data dependencies in question. (See fig. 2.2.)

A CFG is a directed graph describing the possible paths of execution through a program. Vertices in a CFG represent basic blocks, sequences of operations which may only be entered at the head of the block and which may only transfer control at its end. Consequently, if a basic block is entered then all operations within it must be completed prior to control leaving the block. The use of basic blocks instead of single operations for vertices simplifies the CFG; only the potential control branches must be explicitly represented.

While CFGs model the control flows between basic blocks, DFGs model the data flows between operations. Specifically, they model the data dependencies between operations where a directed edge $a \rightarrow b$ designates that $b$ consumes the value computed by $a$.

CFGs and DFGs are often used by other analyses and transformations, such as reachability 


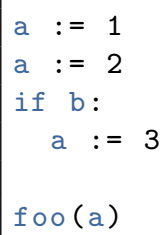

(a) Example program.

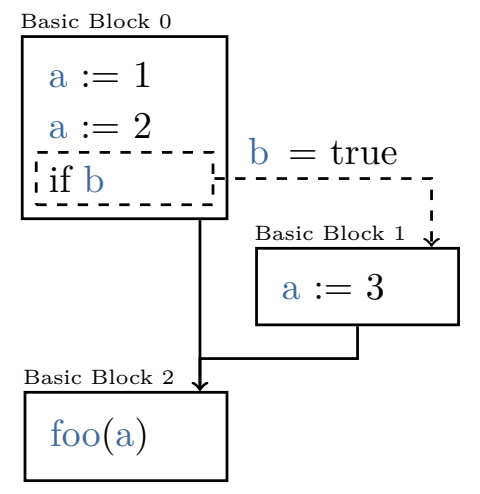

(b) CFG for a.

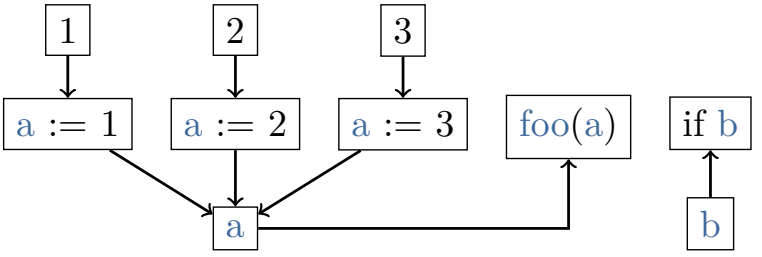

(c) Non-SSA DFG for a.

Fig. 2.2: A program, its CFG, and its non-SSA DFG.

analysis or converting the program into SSA form.

\subsubsection{Single Static Assignment}

Precise data-flow analysis is complicated by situations where a variable, a named store for a value, has more than one assignment. One solution is to convert the program into SSA form where variable is assigned exactly once. This is done by introducing a fresh variable at every assignment, and adding $\phi$-nodes to disambiguate situations where the choice of fresh variable is dependent upon the executed control flow of the program [15, 21, 70]. Figure 2.3 provides an illustration of this conversion.

\subsection{Type Systems}

Many languages offer a type system for verifying a program's data-flow. Broadly speaking, in a typed language program terms are annotated with a type designating a set of permitted values. The program can then be verified (type checked) against these annotations to ensure 


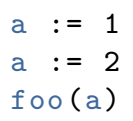

Original form.

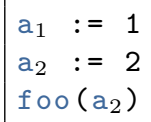

SSA form.

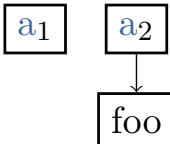

SSA dataflow graph.

(a) An example program without control-dependent data flow.

\begin{tabular}{|l}
$\mathrm{a}:=1$ \\
if $\mathrm{b}:$ \\
$\mathrm{a}:=2$ \\
foo(a)
\end{tabular}

Original form.

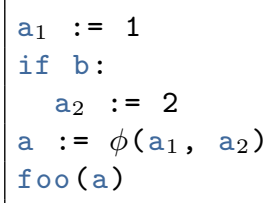

SSA Form

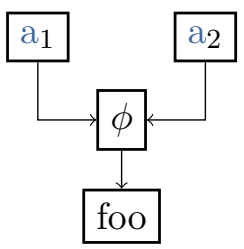

SSA dataflow graph.

(b) An example program containing a control-dependent data flow. $a:=\phi\left(a_{1}, a_{2}\right)$ is inserted to select the correct variable depending on the control flow.

Fig. 2.3: Two programs containing multiple assignments to a variable. While a can be trivially transformed to use two independent variables, b cannot and requires the use of a $\phi$-node.

it does not violate the type system's rules.

While type systems vary in terms of expressiveness and capabilities, the majority guarantee ${ }^{2}$ that a program which successfully type-checks contains no operations using values which are incompatible with the operation's declared type. Type-checking can occur either prior to execution (static) or during execution (dynamic). The details depend heavily on the type system in question, and any guarantees are often contingent upon the program being otherwise well-defined according to the language.

\subsubsection{JVM Type System}

The Java virtual machine (JVM) uses a dynamic type-system designed to map closely onto Java's original static type system $[1,2] .{ }^{3}$ Specifically, the JVM's type system is an example of a nominal subtyping system, where two types are considered identical if and only if they have the same fully qualified name, and types may be declared to be a subtype of one

\footnotetext{
${ }^{2}$ Very few type-systems are formally and comprehensively verified. As a result, it is not uncommon to find type systems or implementations which are unsound. e. g. Java and Scala are both unsound due to an interaction between generics and the bottom value null [7], while TypeScript is deliberately unsound to allow for idioms inherited from JavaScript.

${ }^{3}$ Java's type system was later extended to support parametric polymorphism but this was implemented using type erasure and runtime conversion checks in order to avoid changes to the JVM.
} 
another. The notion of a subtype allows defining non-disjoint sets of values: If $\mathrm{A}$ is a subtype of B (formally written as $A<: B$ ) then the set of values encompassed by $\mathrm{A}$ is subset of values encompassed by its supertype B. Note that the JVM type system permits a type to have multiple supertypes.

\subsection{Neural Networks}

Artificial neural networks (neural networks from this point forward) are a class of parameterized functions inspired by biological neural networks. The objective was to create a system capable of learning from experience much like biological brains. This was accomplished by crudely mimicking the interconnections and non-linear behaviour of biological neurons using scalar weights, and simple non-linear activation functions to approximate the complex stimulus-response behaviour of biological neurons. Neural networks were later proven capable of approximating arbitrary functions [27, 42, 43, 53, 61].

A neural network can be factored into two components: [11]

- Architecture: Network parameters which are defined prior to fitting the network against a dataset, such as the choice of activation function(s), network size, and network connectivity.

- Weights: Network parameters which are obtained while fitting the network against a dataset.

Some architectural parameters (also referred to as hyper-parameters) are chosen by a human, such as the general connectivity structure of the network, while others are tuned to a specific task or dataset using an automated search.

For this review we will be primarily focusing on supervised techniques, where a network is conditioned to emit response $y$ given input $x$, i. e. $y=f_{\phi}(x)$ where $\phi$ is the network's weights. The process of conditioning a neural network's response to input is known as fitting or training, and is typically done by minimizing a function defining the model's prediction 
error (loss). Minimizing the loss function is usually done by iteratively updating the weights using gradient descent along their partial derivatives with respect to the current loss. This approach is commonly referred to as backpropagation [75].

\subsubsection{Activation Functions}

All modern neural models feature a non-linear response to inputs. This is critical, as without non-linear responses increasing the network depth yields no increase in expressiveness. This can be easily proven by the following argument: Consider a trivial linear network of depth $n$ with a single input vector and no biases where the layers are arranged in a single sequence. This network can be expressed as a series of weighed adjacency matrix multiplications, one for each layer, and can be simplified to a single matrix by multiplying the series. Any linear network can be expressed as the sum of such trivial networks, and are thus equivalent to some linear network of depth 1 . Introducing a non-linear transformation between linear transformations solves this issue.

While any non-linear function can be used as an activation function, three are ubiquitous: sigmoid, tanh, and rectified linear (ReLU, rectified linear unit). These will be referred to as $\sigma(x), \tau(x)$, and relu $(x)$, respectively. Figure 2.4 provides a visualization of their activation.

Tanh and ReLU are commonly used as primary activation functions, whilst sigmoid, due to its range of $(0,1)$, is generally used in gating constructs (introduced below in section 2.3.3) and binary logistic regression. For other purposes, tanh and ReLU are generally preferred over sigmoid since sigmoid does not satisfy $0=f(0)$. This property is desirable when using dropout [79] or bias regularization.

In turn, the choice between tanh and ReLU primarily depends on model depth and capacity relative to model size. ReLU is less susceptible to vanishing gradients than tanh, having a linear response for half of its domain, which is particularly desirable for deeper architectures where vanishing gradients often limit the effectiveness of backpropagation-based optimization. However, empirically they offer inferior capacity per hidden unit compared to tanh [32], do not offer a mean zero activation. In practice, the choice of activation matters less than the 


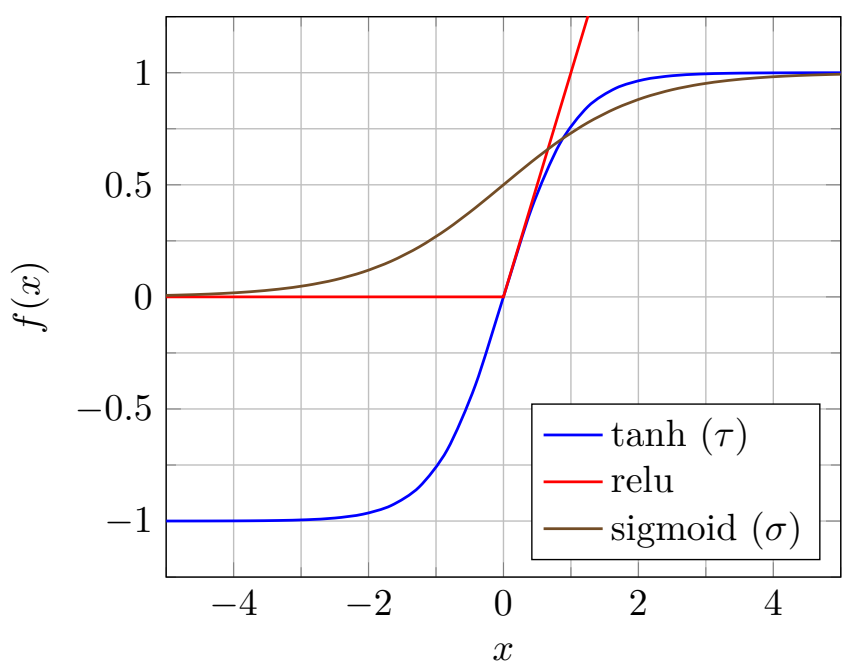

Fig. 2.4: Common activation functions. The sigmoid activation function is defined by $\sigma(x)=\frac{1}{1-e^{-x}}$, and is notable for having a non-zero mean activation.

choice of architecture.

\subsubsection{Feed-forward Networks}

Neural network architectures can be broadly partitioned based on whether their connections form cycles. Those without cycles are termed feed-forward networks, and are generally applied to tasks whose inputs are independent of one another, such as image processing and general classification.

One of the earliest and simplest examples of a neural network is the perceptron (or linear perceptron) [71]. It is a binary classifier and uses a single fully-connected layer (FC LAYER) without any activation function:

$$
f(\mathbf{x})=0<\mathbf{W} \mathbf{x}+\mathbf{b}
$$

The perceptron is only capable of modelling linear functions, and thus can only handle linearly separable datasets. These limitations were solved by the multi-layer perceptron (MLP), which used additional layers of neurons and non-linear activations [75]. The original MLP consists of two fully connected layers, each using a sigmoid activation function. It has been shown that a MLP with at least one hidden layer, i. e. 2 or more total layers, can 
approximate any continuous function [43].

\subsubsection{Recurrent Networks}

Recurrent neural networks (RNNs) extend upon feed-forward networks by incorporating connection cycles, where an output signal is fed back as an input, endowing RNNs with a notion of state. This extension is sufficient to make RNNs Turing-complete [76]. RNNs are commonly used for tasks involving sequences, such as natural language translation and sentiment analysis [17, 81, 82, 93].

A simple recurrent neural network (SRNN) can be defined as:

$$
h_{t+1}=\mathbf{W}\left[x_{t}, h_{t}\right]+b
$$

where $x_{t}$ and $h_{t+1}$ are the inputs and outputs at step $t$ of the sequence.

RNNs are usually fitted using a variant of backpropagation known as backpropagation through time (BPTT) [75]. In BPTT, backpropagation is performed on an 'unrolled' view of the network, allowing the recurrent network to be trained as if it were a feed-forward network with shared weights. In practice the network is only unrolled for a fixed number of steps, rather than the entire sequence. This approach is known as truncated BPTT.

While SRNNs are capable of approximating any dynamical system [27], they are difficult to train using BPTT when pitted against tasks involving long sequences [40, 41]. One popular mitigation is to introduce subnetworks which control how the RNN's state is updated. Two examples of these are Long Short-Term Memory (LSTM) cells [33, 41] and Gated Recurrent Units (GRUs) [19]. Other solutions include using more exotic recurrence transform such as unitary and orthogonal recurrence networks [9, 87].

\subsubsection{Graph Networks}

Neural networks can also be applied to graphs $[23,26,36,55,74,81]$. Common names for these include graphical neural networks, graph convolution networks, message-passing neural 


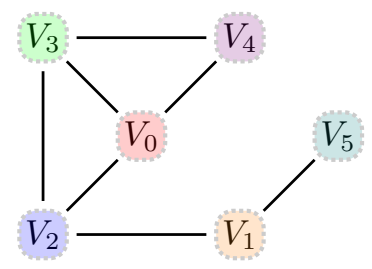

Iteration $i=0: N^{0}(V)$

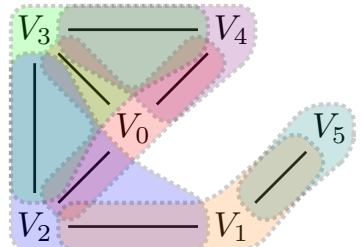

Iteration $i=1: N^{1}(V)$

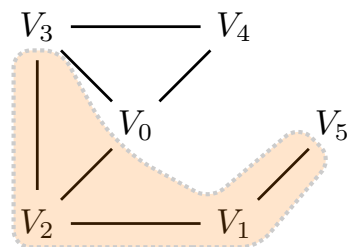

Iteration $i=2: N^{2}\left(V_{1}\right)$

( $V_{1}$ only, for clarity.)

Fig. 2.5: Information is propagated by repeatedly exchanging messages between adjacent vertices in an undirected graph. Displayed here are the first few update iterations. Note that $V_{0}$ and $V_{3}$ are part of $N^{2}\left(V_{1}\right)$, but not the edge $\left(V_{0}, V_{3}\right)$.

networks, and recursive networks (when operating on directed acyclic graphs with a single sink). Graph neural networks are also related to several techniques for learning unsupervised vertex embeddings [34, 63, 66, 95].

To avoid confusion between graph labels (data associated with a vertex or edge) and machine learning labels (ground truth or predicted values), graph labels will instead be referred to as features. Assume some graph $G=(V, E, \mathbf{X}, \mathbf{L})$ where $V$ and $E$ are the vertices and edges, and $\mathbf{X} \in \mathbb{R}^{|V| \times D}$ and $\mathbf{L} \in \mathbb{R}^{|E| \times D}$ are the features for the vertices and edges, respectively. Graph networks are generally of the form

$$
\mathbf{h}_{v}^{i+1}=f_{i}\left(\mathbf{h}_{N(v)}^{i}, \mathbf{X}_{N(v)}, \mathbf{L}_{N(v)}\right)
$$

where $v \in V$ and $f_{i}$ is some parameterized function (possibly using shared parameters for all $i$ ) which updates vertex $v$ 's state based on its closed neighbourhood $N(v)$. The inputs to $f_{i}$ includes the features of incident edges, and the features and states of adjacent vertices. Information is thus diffused through the graph by iteratively updating vertices' states based on their immediate neighbourhood, as illustrated in fig. 2.5. Directional path information can be incorporated by using a non-commutative function for $f$. This process is sometimes referred to as message passing.

Most choices for $f$ can factored into two components: a message-generation function $m$ 
Table 2.1: Examples of previously proposed graph neural architectures, where $f$ has been factored into a message-generation function $m$ and a message-aggregation function $g$ (eq. (2.3)). The choice of initialization for $\mathbf{h}_{v}^{0}$ generally depends on whether $m$ makes use of $\mathbf{X}$. Note that some entries have been simplified for clarity of exposition.

\begin{tabular}{llll}
\hline $\mathbf{h}_{v}^{0}$ & $m\left(\mathbf{h}_{v}, \mathbf{h}_{u}, \mathbf{X}_{v}, \mathbf{X}_{u}, \mathbf{L}_{u \rightarrow v}\right)$ & $g\left(\mathbf{h}_{v}, \mathbf{X}_{v}, \mathbf{M}_{v}\right)$ & Used by \\
\hline $\mathbf{0}$ & $\operatorname{MLP}\left(\mathbf{h}_{u}, \mathbf{X}_{v}, \mathbf{X}_{u}, \mathbf{L}_{u \rightarrow v}\right)$ & $\sum_{\mathbf{m} \in \mathbf{M}_{v}} \mathbf{m}$ & {$[74]$} \\
$\mathbf{W}_{\text {init }} \mathbf{X}_{v}$ & $\mathbf{h}_{u}$ & $\operatorname{GRU}\left(\mathbf{h}_{v}, \sum_{\mathbf{m} \in \mathbf{M}_{v}} \mathbf{m}\right)$ & {$[55]$} \\
$\mathbf{W}_{\text {init }} \mathbf{X}_{v}$ & $\mathbf{W}_{\text {edges }}\left[\mathbf{h}_{u}, \mathbf{L}_{u \rightarrow v}\right]$ & $\operatorname{GRU}\left(\mathbf{h}_{v}, \sum_{\mathbf{m} \in \mathbf{M}_{v}} \mathbf{m}\right)$ & {$[6]$} \\
$\mathbf{W}_{\text {init }} \mathbf{X}_{v}$ & $\mathbf{h}_{u}$ & $\operatorname{MLP}\left(\mathbf{h}_{v}, \frac{1}{\left|\mathbf{M}_{v}\right|} \sum_{\mathbf{m} \in \mathbf{M}_{v}} \mathbf{m}\right)$ & {$[35]$} \\
$\mathbf{W}_{\text {init }} \mathbf{X}_{v}$ & $\mathbf{h}_{u}$ & $\operatorname{MLP}\left(\mathbf{h}_{v}, \max _{\mathbf{m} \in \mathbf{M}_{v}} \mathbf{m}\right)$ & {$[35]$} \\
\hline
\end{tabular}

that operates on incident edges, and a message-aggregation function $g$. In other words:

$$
\begin{aligned}
\mathbf{h}_{v}^{i+1} & =f_{i}\left(\mathbf{h}_{N(v)}^{i}, \mathbf{X}_{N(v)}, \mathbf{L}_{N(v)}\right) \\
& =g\left(\mathbf{h}_{v}^{i}, \mathbf{X}_{v},\left\{m\left(\mathbf{h}_{v}^{i}, \mathbf{h}_{u}^{i}, \mathbf{X}_{v}, \mathbf{X}_{u}, \mathbf{L}_{u \rightarrow v}\right) \mid \forall u \in N(v) /\{v\}\right\}\right)
\end{aligned}
$$

Table 2.1 lists some examples used by existing work. Of these, the GRU-based architectures are the most closely related to the updated function used in this work (see section 4.2).

Graph networks for non-cyclic directed graphs (i. e. recursive networks) typically update each vertex exactly once. By contrast, networks for cyclic or undirected graphs are either run until convergence [74] or use a fixed number of updates [23, 35, 55]. Both have disadvantages. Convergence requires a contractive update function $f$, which excludes many useful architectures (e.g. gating or attention mechanisms), and imparts an exponential decay on the influence of one vertex on another based on their distance [55]. Using a fixed number of update, obviously, limits the maximum distance over which two vertices may interact, but allows for arbitrary neural architectures.

The per-vertex embeddings $\mathbf{h}$ can also be used to produce an embedding for the whole graph. Common techniques include simply aggregating (section 2.4) the embedding (e.g. $\left.\frac{1}{|V|} \sum_{v} \mathbf{h}_{v}^{i}\right)[46$, $55]$, or designating a specific vertex to act as a representative of the entire graph $[26,55,74]$. 


\subsection{Structured Representations}

Representations can be broadly classified as either structured or unstructured. For this thesis, unstructured representations are defined as some embedding $\mathbf{x} \in \mathbb{R}^{n}$ while structured representations are defined as directed graphs whose vertices are labelled by unstructured representations. Undirected graphs, multigraphs, annotated edges, and vertices with structured annotations can all be encoded by introducing additional vertices and edges.

Unstructured representations are typically defined to be (or interpreted as) a sample drawn from some probability distribution. They can be used with practically all common models (support vector machines, graphical models, random forests, etc). By contrast, structured representations are more difficult to handle since a graph may contain an arbitrary number of interdependent unstructured representations. e. g. a sequence might have an unlimited length. Many machine learning models, however, can only handle unstructured inputs.

There are three approaches for dealing with structured representations: one can ignore the connectivity of the graph, one can require that the graph be comprised of uniform local regions, where a region is an induced subgraph of the structured representation; or one can use kernel methods to abstract away how the structure is considered.

The first approach, aggregation, uses the unstructured representations of the graph with no regard to connectivity. This approach is suitable when the input has a variable size and no notion of finite position for the vertices within it (e.g. a set, or unbound sequence). Examples of this class include bag of words, continuous bag of words, multi-instance learning techniques [8], and transformer networks [85]. ${ }^{4}$

The second approach, convolution, considers the local structure of a region within the graph. Unlike aggregation, convolutional approaches consider both a vertex's features and its position within the region in question. ${ }^{5}$ Examples of this approach include convolutional neural networks and graph neural networks.

\footnotetext{
${ }^{4}$ Note that some transformer networks add a periodic feature clock to encode a vertex's position within sequence.

${ }^{5} \mathrm{~A}$ vertex might not have an unambiguous position relative to the entire structure, e. g. cyclic graphs with no sources or sinks.
} 
The third class, kernel methods, entirely side-steps the issue of finding an unstructured reduction. Instead, a kernel function $k: X \times X \rightarrow \mathbb{R}$ provides a similarity score between two samples. This score can be used as a scalar product of some implicit embedding $\psi$ :

$$
k\left(X_{i}, X_{j}\right)=\left\langle\psi\left(X_{i}\right) \cdot \psi\left(X_{j}\right)\right\rangle
$$

Kernel functions can replace scalar products of embeddings in any linear model, and are often used in conjunction with support vector machines (SVMs).

The main drawback of kernel methods for structured representations lies in the computational cost of evaluating the kernel function. Kernel functions based on random walks of vertices matched by sub-kernel functions have a complexity of $O\left(|V|^{2}\right)$, assuming sparse graphs $(|E|=O(|V|))[86]$. For models which require a complete Gram matrix between training and test samples, this corresponds to a total complexity of $O\left(|V|^{2} n m\right)$ for $n$ training and $m$ test samples, where $|V|$ is the largest graph. Faster graph kernels have been suggested, but these require a trade-off in terms of flexibility, such as support for discrete or continuous labels. 


\section{Chapter 3}

\section{Related Work}

The approach proposed in this thesis is based on two ideas: using dependence graphs and graph neural networks to represent programs [6, 31, 46], and classifying (sub)graphs using neural networks $[31,74]$.

This chapter discusses existing works ${ }^{1}$ applying machine learning to programs. This includes works related to defect detection using neural models, techniques for representing programs for neural models (graphical or otherwise), and works concerned with classifying programs or other graphical data.

The first section provides a sampling of various program representations used in recent works, with a focus on techniques suitable for modelling fine grain program properties. e. g. the name of a local variable, rather than the name of a function. The following three sections discusses machine learning based approaches for defect detection. This includes approaches based on supervised classification (section 3.2), detecting "unnatural" outliers (section 3.3), and inferring specifications for formal verification (section 3.4).

Note that many of the works discussed here use unsupervised techniques, and while vast amounts of open source code can be readily obtained, labelled datasets for programs or source code are less common [5, 45, 46, 49, 60, 65, 91]. Finally, the works discussed in this chapter represent a small subset of recent research on probabilistic models of programs and source code. A more general survey of these efforts is available [5].

\subsection{Program Representations}

As discussed in section 2.1, a program can have multiple representations. While that discussion was framed from a compiler's or interpreter's point of view, the same principles apply for

\footnotetext{
${ }^{1}$ Including works concurrent or subsequent to the approach presented here (early 2018).
} 
machine learning: the input representation should be tailored to the task at hand.

Syntactic representations are particularly appealing for machine learning because they are simple, mostly task agnostic, and easy to produce. Their only requirements are a lexer and a parser for the language in question, and that the source code is syntactically valid. In exchange, they offer the equivalents to part-of-speech tagging (tokens) and sentence structure (abstract syntax trees (ASTs)).

Semantic representations offer much more information than syntactic representations [37], but they are also more difficult to obtain. Producing these representations requires prior knowledge of the semantics of the language, and, depending on the semantic analysis in question, other ancillary information (e.g. library dependencies, build configurations, etc).

The most readily available representation, a sequence of lexemes or tokens, is generally a poor one for learning fine grain program properties (e.g. the name or type of a variable). Briefly, programming languages are sensitive to minor lexical changes, grammatically complex with deep and broad syntax trees; and riddled with nonce words. [5, 24, 37] Altering a single lexeme can drastically change the semantics of a program, and the distance between two lexemes in the source code might have little correlation with their distance within the syntax tree. This is further complicated by syntactic sugar (equivalent but distinct syntactic constructions), and the fact that references to a common entity, such as a variable, are often lexically and grammatically distant from one another. Finally, the name of an identifier generally has no effect on the semantics of a program or program element.

Despite all of this, natural source code (i.e. code written by humans) exhibits greater regularity than text drawn from a natural language [28, 39], and even simple lexical representations can be surprisingly effective when augmented with a small amount of semantic or grammatical information.

For example, Allamanis et al. [4] predicted the name of a function given its body using sub-lexemes and an n-gram-like log-bilinear neural model [58]. Their approach indirectly includes grammatical information by only using the lexemes which appear within the body of the function under consideration. 
White et al. [92] approached the task of code clone detection using auto-encoder embeddings of lexical and grammatical contexts, and measuring similarity based on embedding distances.

Explicit combinations of syntactic and semantic representations have also been used. In Allamanis and Brockschmidt [3], variables were represented using an aggregation of their context vectors; a context vector being an embedding of the n-gram centered on the variable's lexeme. This aggregation was done using a recursive network upon the variable's data flow graph, providing the model with semantic information regarding the usage of each variable. Their model inferred which variable should be used for a given context.

A similar model was proposed by Bavishi et al. [13], who inferred the likely names for an identifier given an embedded aggregation of that identifier's n-gram token contexts. These contexts were embedded using a sequence auto-encoder. [22] Like Allamanis and Brockschmidt [3], Bavishi et al.'s approach implicitly included semantic knowledge of the language by recognizing which identifiers refer to the same entity (variable or local function), and aggregating the contexts surrounding these references.

This approach was generalized in Allamanis et al. [6], where programs were represented using a graph consisting of the union of their lexeme sequence, AST, and data-flow edges. This graph was processed using a gated graph sequence neural network[55] to produce embeddings for each node. The nodes' state were initialized using a concatenation of an embedding of their name and the type of value produced. As in Allamanis and Brockschmidt [3], they applied their model to inferring which variable was most likely used in a given context, and demonstrated a significant improvement over the n-gram lexeme context approach. They also evaluated the ability of the model to learn embeddings suitable for inferring the name of a variable given its usage contexts.

By contrast, Raychev et al. [68] proposed a non-neural approach using conditional random fields and a graphical model describing relationships between program properties. These relationships include data flow dependencies and other semantic information. Given a program for which a subset of properties are known, the remaining unknown properties are iteratively assigned their most likely candidate. They demonstrated their approach by 
inferring the names and types of variables in minified source code.

A purely semantic representation was proposed in Henkel et al. [37], where a program is represented using its set of possible traces (execution paths). Each trace is in turn represented as a sequence of semantic events, where an event is some notable action (e.g. function invocation), or a relationship between two or more entities (e.g. two function invocations sharing a common argument). Each distinct event is handled as symbolic value and embedding using GloVe. [62] This approach eagerly decomposes a graphical structure into a set of sequences, simplifying later processing. However, the number of traces for a program can be exponential relative to the number of possible branch points, and nested branch points can induce many distinct traces sharing a common prefix, biasing the embeddings for symbols in the shared prefix.

\subsection{Supervised Classification of Programs}

The most direct approach for learning defect detection is to handle it as a supervised classification problem. Supervised modelling of programs, or structured data in general, is not a new idea, and has a history tracing back to heuristics for Checker playing programs [73] and theorem provers $[25,30,80]$. The main complication is dealing with the structured nature of programs. This requires both selecting an appropriate representation for the program (section 3.1), and a decision regarding the granularity of the classification: should the program be classified as a whole, or should each part of the program be assigned a classification? The latter is more general, but may be more expensive and difficult to train.

Several recent works on defect detection use unstructured representations and most use representations implicitly capturing structured information. Examples of these works include syntax-derived embeddings used to infer both very coarse (per class or file) classifications [54, 88], and fine grain (per token) classifications [65, 72]. These efforts are only cursorily mentioned here since using unstructured representations shifts the responsibility, and complexity, of handling the program's structure from the model to the representation extraction (see section 3.1). 
Early neural models for program classification made use of vertex auto-encoders and recursive networks $[31,78]$. Toy benchmark used in these experiments involved classifying lambda calculus expressions based on the presence of specific sub-expressions. The core idea was to represent a program (e.g. a lambda expression) as a directed graph, and use its topology to define a graph neural network. $[26,31,77,78]$ Modern variants of this idea have been used to learn heuristics for selecting promising theorems [46], estimating the throughput of basic blocks [56], and learning the most likely return codes for function traces [37]. Lastly, neural networks have also been used for detecting specific sub-graph isomorphisms [74]. While not directly a program classification task, it bears mention as it is directly related approach proposed in this thesis.

\subsection{Outlier-based Defect Detection}

Given that natural source code is regular and repetitive [28, 39], and the (perhaps optimistic) assumption that the majority of code is non-defective, one approach for detecting defects is to simply identify "unnatural" code. Ray et al. [67] provide a direct example of such an approach. Using a lexeme n-gram based model, they show that examining lines of code ranked by perplexity can be cost-competitive with conventional static analyzers.

Henkel et al. [37] performed a similar exercise, where they trained a Long Short-Term Memory (LSTM) model to predict the most likely return code for a given trace. The fitted model was evaluated against real world defective traces, and in the majority of cases the defective return code was not within the top-3 predictions issued by the model.

Non-probabilistic models have also been applied to code anomaly detection. Wasylkowski et al. [89] data mined function invocation sequences and proposed a scoring method for quantifying a sequence's abnormality. When applied to existing software, their approach identified several defects, ranking them among the top-10 most anomalous sequences. Monperrus and Mezini [59] proposed a related, but more specialized, approach focused on identifying erroneously omitted invocations. 


\subsection{Specification Inference}

An alternative approach to defect detection is to infer a specification rather than directly attempt to rank or classify a sample as unnatural or defective. The inferred specifications can then be used to deterministically verify programs. These techniques overlap with other approaches based on identifying unnatural code, but the primary distinction between them lies in the explicability of their results: a specification is a white-box model and can be systematically analysed; by comparison, discerning why a given sample is highly perplexing for a black box model can itself be a rather perplexing exercise for the examiner [47].

Kremenek et al. [50] and Banerjee et al. [12] both used factor graphs [96] and a set of relationship constraints to infer semantic roles for each function in a program. In Kremenek et al. [50] these roles modelled resource ownership, and were used to identify resource ownership violations (e.g. leaks, or double-releases). In Banerjee et al. [12] these roles consisted of tainted (adversary controlled) data sources, sanitizer, and vulnerable sinks (data consumers). A source directly connected to a sink without an intermediary sanitizer is considered a defect.

More general kinds of specifications can also be produced, such as separation logic formulas for describing heap structures [69]. Brockschmidt et al. [16] produced separation formulas using hand crafted features describing a graph structure. These hand crafted features were replaced in Li et al. [55] by a graphical neural network, which achieved superior accuracy with minimal manual feature engineering. 


\section{Chapter 4}

\section{Model}

The objective of thesis is to develop a method for learning to identify defects from sourceline annotated examples. The proposed approach is to learn which partial subgraphs in a program's dependence graph, a directed graphical representation of the program's computation dependencies, are correlated with a defect. By considering dependencies between operations (such as subroutine invocations), the proposed approach is particularly suited for identifying defects caused by improper application programming interfaces (APIs) usage. The scope of these graphs is limited to individual subroutines, rather than the entire program, in order to keep their size tractable.

The problem of identifying a specific subgraph is approached bottom-up. Given a vertex $v$ from some graph, we wish to infer the likelihood that $v$ is part of a defect-indicating subgraph based on its $k$-neighbourhood, $P\left(v \mid N^{k}(v)\right)$, where $k$ is a hyper-parameter and $0 \leq k$. The choice of $k$ determines the computational cost of the model and the size of defect subgraphs the model is capable of precisely classifying. Namely, to precisely classify the vertices of a defect subgraph with diameter $d, d \leq k$ is necessary.

The proposed approach can be summarized as follows: Assume a dependence graph $G=$ $(V, E, X, L)$, where $X$ and $L$ are the features for the vertices and edges, respectively. The vertex features $X$ are used initialize a graph neural network, and the network is updated for $k$ steps to produce a latent embedding for each vertex incorporating information from connected vertices up to $k$ steps away. These latent embeddings are used as embeddings of the vertices' $k$-neighbourhoods. Finally, vertices are independently classified using the concatenation of their features with their final graph network state. A depiction of this model is provided in fig. 4.1.

The graphical representation and features is described in section 4.1, followed by the $N^{k}(v)$ embedding graph neural network in section 4.2, and the objective function for the model 


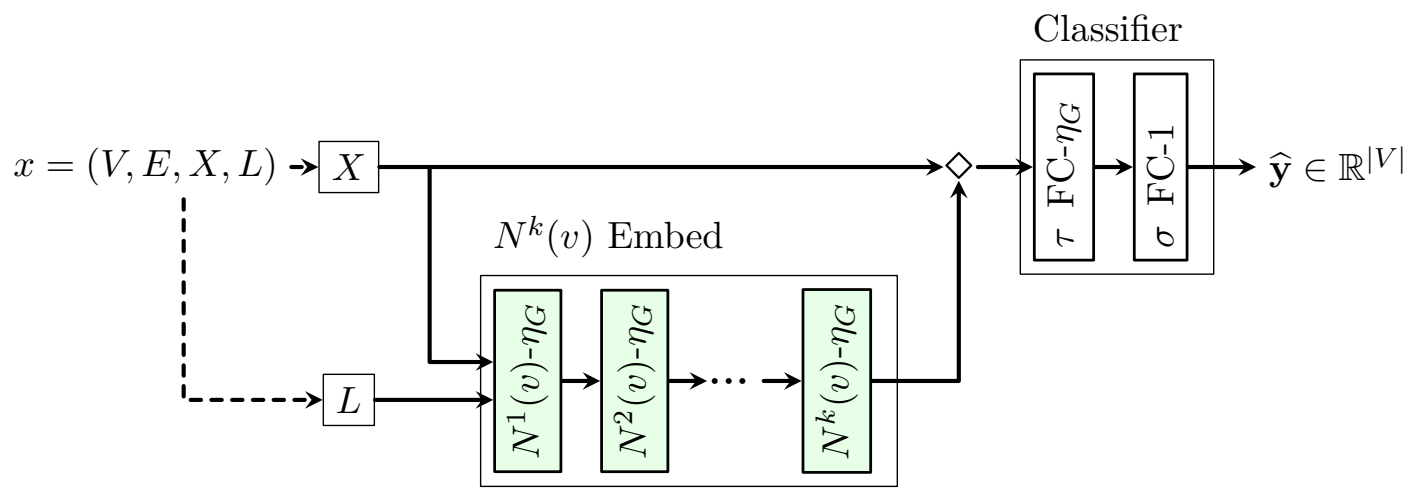

Fig. 4.1: Overview of the proposed classifier. $\diamond$ designates concatenation of feature vectors, FC- $n$ is a fully-connected layer with $n$ outputs, and $\eta_{G}$ is a hyper-parameter specifying the width of the $N^{k}(v)$ embeddings. Layers with a common background colour shared weights.

(section 4.3). For clarity of presentation, the exact values for hyper-parameters selected via hyper-parameter search are defined in the next chapter, in section 5.2.1.

\subsection{Representation}

In this work, a subroutine is represented by a dependence graph, a directed multigraph where the subroutine's operations are vertices, and the dependencies between these operations are edges. Vertices and edges are labelled with symbolic (categorical) features describing their actions and operands. Some have pre-determined vocabularies, while others have datasetdefined vocabularies where the vocabulary is specified during fitting. Table 4.2 provides a summary listing of these features.

These representations are primarily produced using Soot [84], a Java byte-code optimization library. It is used to parse Java virtual machine (JVM) byte-code, perform basic semantic analysis, and apply basic compiler optimizations. The representations are derived from their single static assignment (SSA) intermediate representation (IR), Shimple, and each operation generally corresponds to a Shimple instruction. 


\subsubsection{Dependence Graphs}

A dependence graph is a directed multigraph where vertices represent operations performed by a program and edges designate dependencies between operations. In principle, these dependencies should include control dependencies for sequencing operations with sideeffect. However, I make a simplifying assumption that all operations are pure functions. This allows us to ignore control dependencies, which significantly reduces the graph connectivity, incidental neighbourhood diversity, and potential for overfitting. However, this simplification does preclude learning precise discriminators for defects dependent on specific side effect sequences.

Dependence graphs are produced by loading the target program in Soot, applying several basic compiler optimizer with the goal of reducing the program size by eliminating simple redundancies (table 4.1), and extracting the SSA data-flow graph (DFG) of the optimized subroutines. No interprocedural analyses or optimizations are used. A second pass of ad-hoc transformations is then applied to the extracted graphs to remove some uninformative artifacts (see section 4.1.2).

An example of a dependence graph and its associated source code is displayed in fig. 4.2. In this example, two related defects have been identified and their inducing lines marked as defective. The first defect is an Structured Query Language (SQL) injection vulnerability, while the second involves reading from a source of potentially tainted data without sanitation.

\subsubsection{Graph Simplification}

The graphs produced from Soot contain a number of incidental artifacts which are uninformative to a discriminative model. Examples include certain instructions which are (almost) always produced in pairs, explicit $\phi$-nodes, and the use of temporary variables for storing the results of sub-expressions, even in situations where there is a single consumer for the result.

Most of these artifacts can be eliminated by applying the following transformations: 
Table 4.1: Optimizations performed using Soot, applied in order from top to bottom. None are esoteric and they represent the minimum one would expect from an optimizing compiler.

\section{Soot Optimization Passes}

\section{Nop Elimination}

Removes no-op instructions

\section{Constant Propagation}

Simplifies indirect references to constants (e.g. $a=5 ; b=x \rightarrow a=5 ; b=5$ )

\section{Copy Propagation}

Simplifies indirect references to variables (e.g. $\mathrm{a}=\mathrm{x} ; \mathrm{b}=\mathrm{x} \rightarrow \mathrm{a}=\mathrm{x} ; \mathrm{b}=\mathrm{x}$ )

\section{Constant Folding}

Constant expressions are immediately evaluated (e.g. $1+1 \rightarrow 2$ )

\section{Common Sub-expression Elimination}

Common sub-expressions are evaluated once

(e. g. $a=(x+1) *(x+1) \rightarrow b=x+1 ; a=b * b ;)$

\section{Dead Assignment Elimination}

Assignments which have no possible reads are removed

\section{Unused Local Elimination}

Unused variables are removed

\section{Unconditional Branch Folding}

Simplifies branches which always occur (e.g. if (true) $\{\ldots\}$ )

\section{Unreachable Code Elimination}

Removes code which could never execute (e.g. if (false) $\{\ldots\}$ )

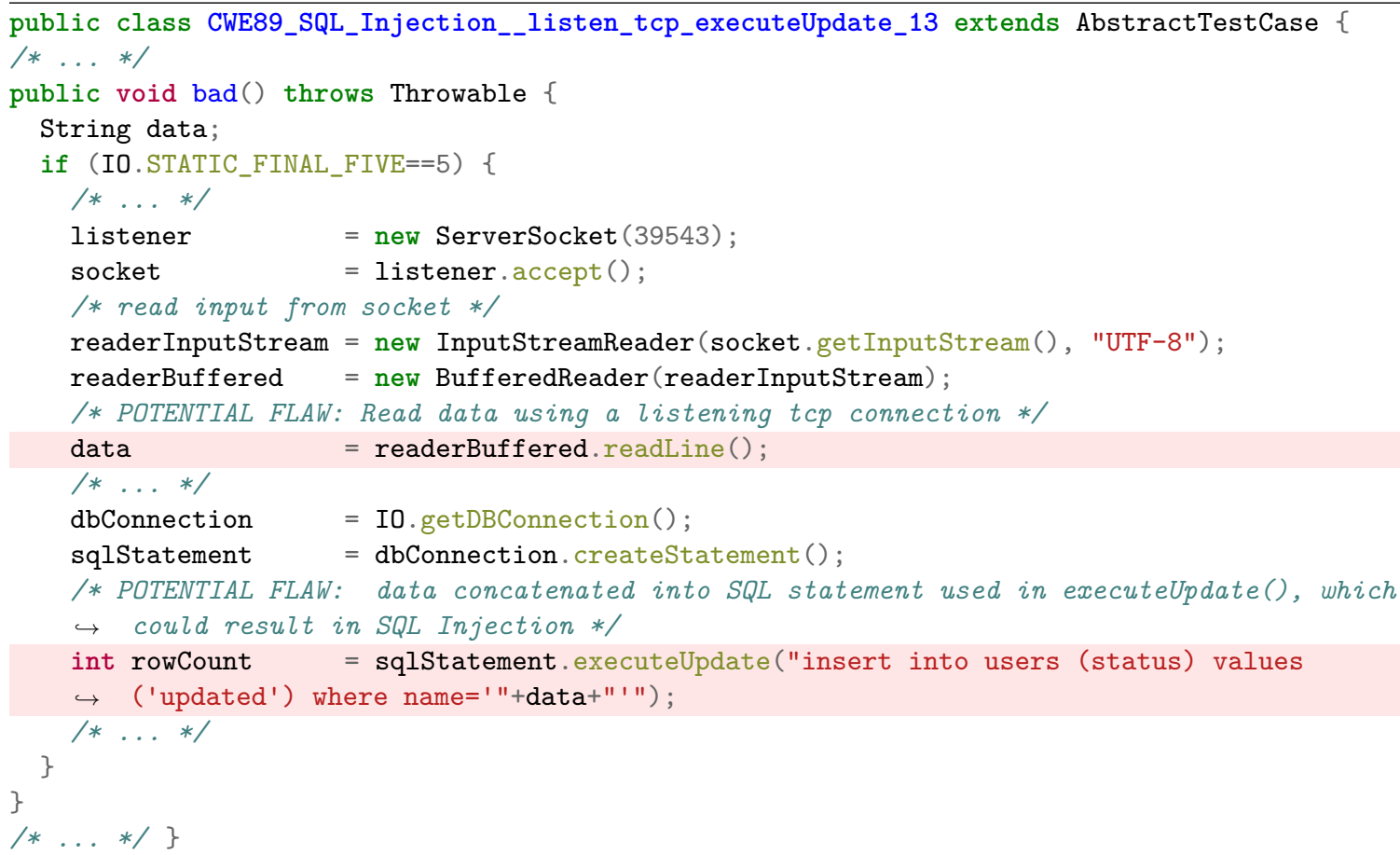

(a) Source code excerpt from a Juliet test case (section 5.1.1). Ancillary lines are omitted. 
롱

$\sum_{\text {M. }}^{2}$

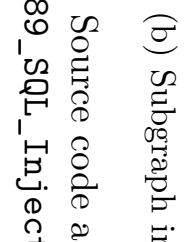

岱.

哭

15. 8

की

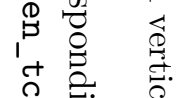

i

$\begin{array}{lll}1 & \infty & 0 \\ 1 & 0 \\ 14 & 0 \\ 0 & 0 & 0 \\ 0 & 0\end{array}$

足

怘若

प्वे क्ष

$\begin{array}{lll}0 & 0 \\ 0 & 0 \\ 0 & 0 \\ 0 & 0\end{array}$

岕密索

苗. 武.

总

旁

ॐ

की

氮

宊

索

苛

疋

苋

号

ऽ

․ㅗㅇ

or $E$.

$\dot{0}$

क्ञ

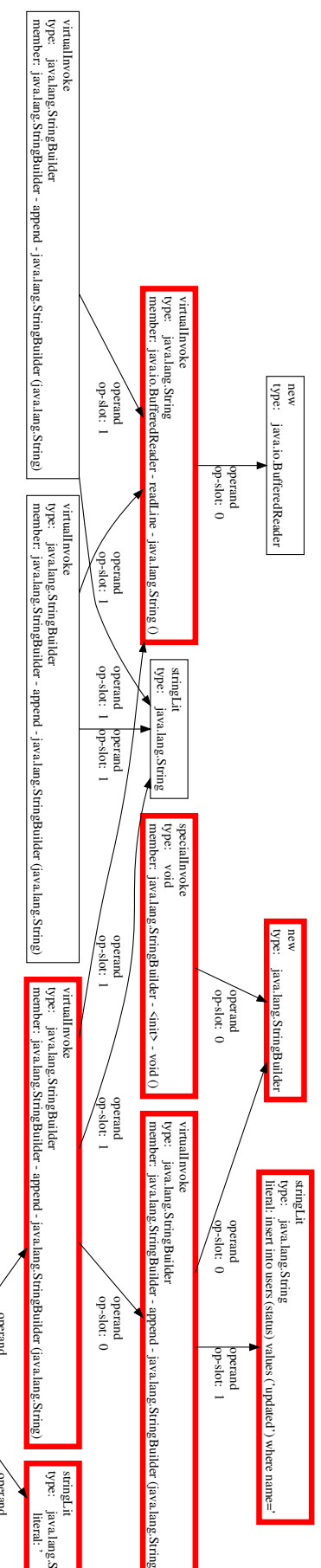


i. Fold statement place-holders into their hosted expression. (e.g. invoke-statement.)

ii. Fold local variables onto their right-hand-side expression (RHS) expressions. Since Shimple is an SSA representation, all variable have exactly one RHS expression.

iii. Replace dependencies onto a $\phi$-node by dependencies onto the $\phi$-node's transitive dependencies. Any duplicate dependencies are discarded, and all $\phi$-nodes are erased.

iv. Remove null-literal arguments for parameter 0 of non-static functions; such invocations can never succeed at runtime. This is a common artifact caused by a $\phi$-fold referencing a null-initialization. If the $\phi$-node was transitively dependent on a pre-SSA variable that was reused between otherwise independent sections of code then this can result in significant and spurious connectivity between those sections' connected components within the dependence graph.

An example of a fictional graph being simplified using transformations ii. and iii. is provided in fig. 4.3.

It is important to note that these simplifications generally do not introduce or remove information from the graph. The erased vertices and edges provide no information since their features can be precisely and unambiguously inferred from neighbouring vertices. By removing these uninformative vertices the graphs become less computationally expensive and larger neighbourhoods can be covered for the same number of message passing iterations.

On the Juliet dataset (section 5.1.1) these simplifications reduce the mean vertices per sample from 236.84(145.33) to 29.94(46.5), while on the natural-negative dataset (section 5.1.2) it reduces the mean vertices per sample from $40.09(282.97)$ to $19.36(144.05)$.

\subsubsection{Vertex \& Edge Features}

Individual operations and dependencies are described using the features listed in table 4.2. The following subsections describe each feature's purpose and how they are embedded. Not all features necessarily apply to a given operation or dependence, and some features are 


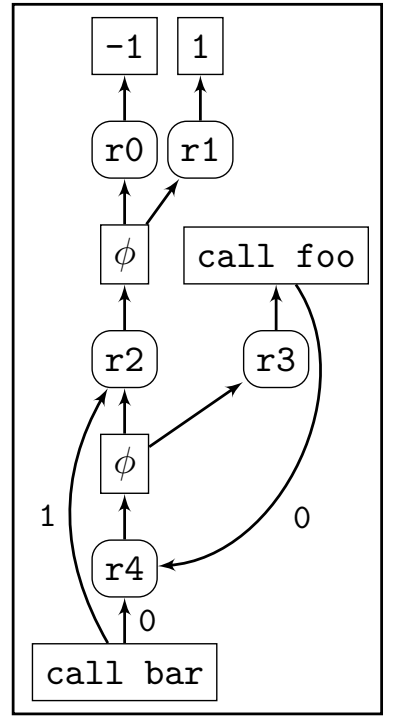

Original graph.

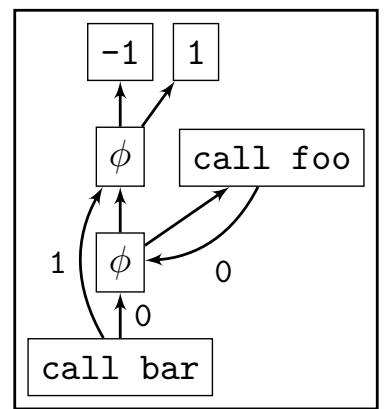

ii. Fold RHS onto locals.

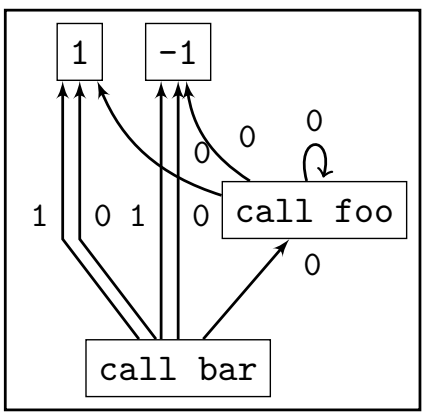

iii. Fold $\phi$ dependencies.

Fig. 4.3: Examples of RHS and $\phi$-dependence simplification (items ii. and iii.). Edge labels denote the operand indices, if applicable. Note the multiple edges in iii..

Table 4.2: List of operation and dependence features.

\begin{tabular}{rrrl}
\hline Host & Feature & Type & Description \\
\hline Operation & Instruction & Categorical & Action performed by operation. \\
Operation & Member & Categorical & Any referenced member. \\
Operation & Result Type & Set of Categorical & The operation result's type. \\
Operation & Const. Value & Sequence of Categorical & Any referenced constant value. \\
Dependence & Dep. Type & Categorical & The type of dependence. \\
Dependence & Operand Index & $\{x \mid x \in \mathbb{Z}, 0 \leq x\}$ & Operand's argument index. \\
\hline
\end{tabular}

conditional upon others. A special non-applicable symbol is used whenever a specific feature does not apply. Note that this symbol is distinct from the out-of-vocabulary (OOV) symbol.

\subsubsection{Operation Features}

An operation $v$ is described by the tuple $X_{v}=\left(X_{v}^{(\mathrm{I})}, X_{v}^{(\mathrm{M})}, X_{v}^{(\Gamma)}, X_{v}^{(\mathrm{C})}\right)$, whose components are the operation's instruction, member reference, result type, and constant value, respectively. $X^{(\mathrm{I})}$ and $X^{(\mathrm{\Gamma})}$ are defined for all operations. $X^{(\mathrm{M})}$ and $X^{(\mathrm{C})}$, however, are only defined for operations with certain instructions. e.g. an operation with a numeric literal instruction must also have a constant value and cannot reference any members. 
Note that the symbol vocabularies for $X^{(\Gamma)}$ and $X^{(\mathrm{M})}$ are dataset-defined and are not known prior to fitting.

Instruction $\left(X^{(\mathrm{I})}\right)$ : Specifies the fundamental action performed by an operation. e. g. branch, invocation, addition. This particular implementation uses Shimple's instruction set. Note that unlike JVM byte-code instructions, Shimple instructions are polymorphic, e. g. there is a single instruction for addition for all argument types, and do not use special instructions for common constant values.

This feature uses a predefined-defined symbol vocabulary, and is embedded using a one-hot vector.

Member $\left(X^{(\mathrm{M})}\right)$ : Specifies which, if any, member is referenced by an operation. A member is either a function or a field of an aggregate type. Member symbols consist of their fully qualified name at their point of initial declaration.

For fields, this makes no difference (they can only be declared once), but for methods this distinction is important, as dynamic dispatch is equivalent to invoking functions stored as values within fields. For example, consider some defect which only occurs given the invocation of a specific override of an abstract method. Given a program where the abstract method is invoked using dynamic dispatch, it is impossible to know which override should be invoked without knowing the object's precise type. While this information is available at runtime, it cannot always be precisely determined using static analyses. In a dependence graph, the $X^{(\Gamma)}$ feature of a dynamic dispatch's first argument (i. e. the dispatching object) can be used to infer a lower bound on the set of overrides which could be invoked.

This feature uses a dataset-defined symbol vocabulary, and uses learned embeddings of width $\eta_{E} \cdot$

Type $\left(X^{(\Gamma)}\right)$ : Specifies the type of produced by an operation. The type void is used for operations which have no result value. Type symbols consist of their type's fully qualified name.

Subtyping relationships are modelled using the approach proposed in Allamanis et al. [6], 
where a type $t$ is represented by a set of all its supertypes:

$$
\begin{gathered}
\Gamma_{t}= \begin{cases}t & t \in \Gamma \\
\text { OOV } & \text { otherwise }\end{cases} \\
S(t)=\left\{\Gamma_{u} \mid \forall u . t<: u\right\}
\end{gathered}
$$

where $\Gamma$ is the model's type vocabulary. Unlike Allamanis et al. [6], the type arguments of type constructor is not include in this set, as the JVM's type system does not support type constructors. ${ }^{1}$

One advantage of using a set-of-supertypes representation is that novel types encountered during testing need only be partially OOV if any of its supertypes were seen during fitting. Similarly, it is possible for an OOV member to have a (partially) known type, e. g. an OOV subroutine which returns a hash map (or some other common type).

This feature uses a dataset-defined symbol vocabulary, and each type in $\Gamma$ uses a learned embedding of width $\eta_{E}$. This feature itself, however, is a set of supertypes. To capture the notion of set membership each supertype is embedded and the set is reduced by taking the element-wise maximum of its members [6].

During training, a modified variant of dropout is applied where members are randomly dropped from the set, ensuring that each member embedding has the chance to receive some measure of fitting $[6,44]$. The technique is similar to regular dropout [79], except that the entire member is removed from the set, rather than setting a random subset of the member's features to zero. The dropout probability is a tuned hyper-parameter, see section 5.2.1.

Note that this naive dropout approach can result in sets describing absurd type hierarchies. Namely, the presence of some type $t$ does not guarantee that all of $t$ 's supertypes are also present. This is not a major concern in practice, but a proper approach would ensure that all subtypes of a dropped type are themselves dropped in turn.

\footnotetext{
${ }^{1}$ Languages supporting type constructors use type erasure and dynamic type casts at the use-sites when targeting the JVM.
} 
Table 4.3: List of string representations for numeric constants. Rational numbers are represented using whichever format is shortest.

\begin{aligned} & \hline Numeric Constant Representation Formatting \\ & \hline Integral Decimal \\ & Rational Decimal or exponential \\ & Infinite "inf", "-inf" \\ & NaN "NaN" \\ & \hline\end{aligned}

Constant Value $\left(X^{(\mathrm{C})}\right)$ : Specifies which, if any, constant value is used by an operation. Constant values are limited to scalars and string literals.

All constants are represented as sequences of Unicode Text Format 8-bits (UTF-8) symbols; numeric constants are formatted as specified in table 4.3.

Constant values are reduced to an embedding $\mathbf{x} \in \mathbb{R}^{\eta_{E}}$ using a stack of convolution layers, rather than a recurrent network. This is primarily motivated by speed and simplicity concerns $[14,48,82,94] .^{2}$ The sequence of characters is first truncated or null-padded to a fix length $2^{n}$, and each character projected using a learned embedding of width $\eta_{E}$. The sequence of dense embeddings is then reduced using a stack of $n$ 1d convolutions, where each convolution uses a window and stride of 2 , halving the the sequences at each layer. This encoder network is admittedly ad-hoc, but fast and efficient.

For the datasets used in chapter 4 (section 5.1), $n=7$ is more than sufficient since as the mean length for a literal is 23.4(15.5) elements. ${ }^{3}$

\subsubsection{Dependence features}

A dependence $e=a \rightarrow b$ is described by $L_{e}=\left(L_{e}^{(\mathrm{Q})}, L_{e}^{(\mathrm{O})}\right)$, where $L_{e}^{(\mathrm{Q})}, L_{e}^{(\mathrm{O})}$ are $e$ 's dependence type and operand index, respectively.

Dependence Type $\left(L^{(\mathrm{Q})}\right)$ : Specifies the type of dependence between two operations. A listing of all dependence types is provided in table 4.4. This is a symbolic feature with a

\footnotetext{
${ }^{2}$ A bidirectional Long Short-Term Memory (LSTM) was initially used, but later abandoned as the computational overhead was unacceptable.

${ }^{3}$ This excludes the 100 largest outliers $(\approx 0.01 \%)$ as these are abnormally large, ranging in length from 470 to 48548 . These outliers include command line help strings, documentation, and encoded binary blobs (lexer and parser tables, public keys, etc).
} 
Table 4.4: List of dependence types.

\begin{tabular}{rc}
\hline Dependence Type & Operand Index \\
\hline Assignment & \\
Index Base & \\
Index Expression & \\
Operand & $\checkmark$ \\
\hline
\end{tabular}

predefined dictionary of length 4 . It is embedded as a one-hot vector.

Operand Index $\left(L^{(\mathrm{O})}\right)$ : Designates to which parameter an argument is applied. The index is zero based, and the 'self' argument of a method invocation uses index 0 by convention.

This feature is embedded as a one-hot vector of length 16. Operand indices above 15 are clamped to 15. While its domain is, in principle, unbound, in practice there are extremely few functions use more than a dozen parameters. The choice of 16 is arbitrary but is more than sufficient for non-contrived functions. ${ }^{4}$

\subsection{Neighbourhood Embedding}

The embedding $\mathbf{h}_{v}^{k}$ of operation $v$ 's $k$-neighbourhood $N^{k}(v)$ is generated using a graph neural network with a gated update function similar to a Gated Recurrent Unit (GRU). Assume a multi-digraph $G=(V, E, X, L)$ consisting of the set of vertices $V$, the directed edges $E$, and the vertex and edge features $X$ and $L$. The update equations for this network are

$$
\begin{aligned}
& \mathbf{m}_{e_{i j} \in E}^{t}=\tau\left(\mathbf{W}_{m}\left[L_{e}, \mathbf{h}_{i}^{t}\right]+\mathbf{b}_{m}\right) \quad(4.3 a) \quad \mathbf{z}_{v}^{t}=\tau\left(\mathbf{W}_{z} \sum_{e_{i v} \in E} \mathbf{m}_{e_{i v}}^{t}+\mathbf{b}_{z}\right) \\
& \mathbf{u}_{v}^{t}=\sigma\left(\mathbf{W}_{u}\left[\mathbf{h}_{v}^{t}, \mathbf{z}_{v}^{t}\right]+\mathbf{b}_{u}\right) \quad(4.3 c) \quad \mathbf{h}_{v}^{0}=\mathbf{W}_{\text {init }} X_{v} \\
& \mathbf{r}_{v}^{t}=\sigma\left(\mathbf{W}_{r}\left[\mathbf{h}_{v}^{t}, \mathbf{z}_{v}^{t}\right]+\mathbf{b}_{r}\right) \quad(4.3 e) \quad \mathbf{h}_{v}^{t+1}=\left(1-\mathbf{u}_{v}^{t}\right) \odot \mathbf{h}_{v}^{t}+\mathbf{u}_{v}^{t} \odot\left(\mathbf{z}_{v}^{t}+\mathbf{r}_{v}^{t} \odot \mathbf{h}_{v}^{t}\right)
\end{aligned}
$$

Equation set 4.3: Update equations for $N^{k}(v)$ embedding network. where $\mathbf{h}_{v \in V} \in \mathbb{R}^{k \times \eta_{G}}$, and both $k$ and $\eta_{G}$ are hyper-parameters.

\footnotetext{
${ }^{4}$ For the primary dataset defined in section 5.1, the mean operand index for operand dependencies is $0.722(0.95)$ and the max index is 22 .
} 
The initial state of a vertex $v, \mathbf{h}_{v}^{0}$, is initialized based upon its features $X_{v}$. The learned projection $\mathbf{W}_{i}$ allows varying the symbol embedding width $\eta_{E}$ and the $N^{k}$ embedding width $\eta_{G}$ independently of one another. If the choice of hyper-parameters results in $X \in \mathcal{R}^{|V| \times N}$ and $N \leq \eta_{G}$ then $X$ is simply zero padded to $\mathcal{R}^{|V|_{G}}$. Message passing is performed for $k$ steps to produce the sequence $\left(\mathbf{h}_{v}^{0}, \mathbf{h}_{v}^{1}, \ldots, \mathbf{h}_{v}^{k}\right)$. The final step, $\mathbf{h}_{v}^{k}$, is concatenated with $X_{v}$ and feed through a multi-layer perceptron (MLP) for classification.

Note that information only flows along the directed edges of the graph. Consider an acyclic graph $G$ and its sources $V_{\text {src }}=\{v \mid v \in V, \forall x \in V .(v \rightarrow x) \notin E\}$. If one were to assemble $N^{i}(v)$ based on outbound edges then $\forall v \in V_{\mathrm{src}}, 0 \leq i . N^{0}(v)=N^{i}(v)$. The same general problem extends to non-source vertices. $G$ is therefore augmented with its reversed-dependence edges in order to include the dependants of $v$ in $\mathbf{h}_{v}$. These reversed edges are marked as such by using reversed variants for their Dependence Type feature. e. g. An operand edge $a \rightarrow b$ induces a reverse-operand edge $b \rightarrow a$. Reverse-dependence edges are excluded from all figures in the interests of visual clarity.

Messages are aggregated by summation eq. (4.3b), although it would be preferable to aggregate them using their element wise maximum. While counting aggregation (summation) subsumes presence aggregation $(\max )$, our task only requires the latter as defects are generally dependent only on the presence of specific symbol paths. Preliminary experiments showed that max-aggregation provides a modest improvement over summation, though in exchange for a substantial increase in memory and computation during training.

This architecture differs from a classic GRU in that it handles the recurrence term as a gated residual-like connection. The recurrence term $\mathbf{h}_{v}^{i}$ is not projected, and it is used in eq. (4.3f) rather than as an input to the activation function. Moving $\mathbf{h}_{v}^{i}$ outside of saturating activation avoids hidden unit saturation (typically mitigated in GRUs by the projection), which in turn reduces gradient attenuation for earlier states. The resulting design bears notable similarity to chaos-free recurrence cells [51], though it inherits an activation bias and double-gated resets from GRUs.

These alterations from a classic GRU are motivated by two factors. Firstly, updating a vertex 
$v$ with an $N^{k}(v)=\{v\}$ should, intuitively, be an idempotent action. Formally, I define this to mean that the sequence $\left(\mathbf{h}_{v}^{0}, \mathbf{h}_{v}^{1}, \ldots\right)$ should be element-wise monotonic. Secondly, delayed recurrence-like connections are implicitly formed by the addition of reverse-dependence edges. Note that removing the input recurrence from eq. (4.3b) is technically insufficient for idempotent updates of edgeless vertices due to possible interactions between $\mathbf{b}_{z}$ and the recurrence connections in $\mathbf{u}_{v}^{t}$ and $\mathbf{r}_{v}^{t}$ (an example is provided in eq. (A.1)). In practice the fitted cells exhibit consistent $\mathbf{h}_{v}$ sequences when driven with null inputs, despite this potential interaction. Further information on this topic, including $\mathbf{h}_{v}$ transition plots, is provided in Appendix A.

\subsection{Objective Function}

The model is fitted by minimizing the vertices' cross-entropy. Assume a labelled dataset $\mathcal{D}$ where $(x, \mathbf{y}) \in \mathcal{D}, x=(V, E, X, L)$, and $\mathbf{y} \in\{0,1\}^{|V|}$. Let the model prediction be $\widehat{\mathbf{y}}$. The objective function is

$$
\begin{aligned}
\mathcal{L}(\theta, \phi) & =\sum_{((V, E, X, L), \mathbf{y}) \in \mathcal{D}} \sum_{v \in V} H\left(\mathbf{y}_{v}, \widehat{\mathbf{y}}_{v}\right)+\lambda \mathcal{R}\left(X_{v}, \theta\right)+\lambda\|\phi\|_{2} \\
\mathcal{R}\left(X_{v}, \theta\right) & =\sum_{X_{v}^{(\Gamma)}<: u}\left\|\theta_{u}^{(\Gamma)}\right\|_{2}^{2}+\left\|\theta_{X_{v}^{(\mathrm{M})}}^{(\mathrm{M})}\right\|_{2}^{2} \\
\widehat{\mathbf{y}}_{v} & =\operatorname{MLP}\left(\mathbf{h}_{v}^{k}, X_{v}\right)
\end{aligned}
$$

where $H(y, \hat{y})$ is the binary cross-entropy between a ground truth label $y$ and a predicted

label $\hat{y}, \theta_{j}^{(i)}$ is the learned embedding for symbol $j$ of symbol vocabulary $i$ (e. g. the Types symbol boolean), and $\phi$ consists of all other model parameters. The only unusual aspect of this loss function is the regularization term Equation (4.5), which imparts a term-frequency loss penalty on the learned embeddings for dataset-defined symbols.

Training and inference procedures for this model are effectively the same as those for a standard feed-forward network. Parameter optimization is done using stochastic gradient descent with RMSProp [83]. Since the number of message passing iterations, $k$, is a hyper- 
parameter constant, the neighbourhood embedding network stack (the $N^{k}(v)$ block in fig. 4.1) can be implemented by simply unrolling the sequence. 


\section{Chapter 5}

\section{Experiments}

The proposed approach is evaluated using various software projects and a number of test suits, as well as its ability to transfer to unseen projects.

Most experiments report two sets of metrics: vertex-level, and sample-level. These correspond to two different scoring methodologies. Vertex-level metrics represent the ability of the model to classify fine-grain details within a sample, and are applied to each vertex within a sample. Sample-level metrics represent the ability of the model to accurately classify a sample with no regards as to fine-grain accuracy. These metrics are applied to each sample as a whole, rather than its vertices. A sample's ground truth and predicted likelihood is the maximum of its vertices', i. e. it is defective if any of its vertices are defective. Sample-level metrics are expected to be much easier to learn, and offer a good proxy metric

Details regarding datasets are presented first, followed by model hyper-parameters and evaluation results. More information regarding the various metrics used in these experiments can be found in Appendix C.

\subsection{Datasets}

To the best of my knowledge, there exists no standard benchmark dataset for identifying specific expressions within a realistic program. I therefore assembled a new using open source projects and static analysis test suites. These projects can be categorized as either synthetic, natural-negative, or natural-defective, depending on the nature of their code. The size and usage of these categories is listed in table 5.1. Projects belonging to the synthetic and natural-negative were combined to produce the primary dataset used for training and testing. This dataset was partitioned 0.6/0.1/0.3 for training, development, and evaluation, respectively. By contrast, projects in the natural-defective category were only used for 
Table 5.1: Population and contents of sources used for the datasets. Despite its name the natural-defective dataset is expected to contain a mix of positive and negative samples.

\begin{tabular}{rrrrrr}
\hline Project Category & \# of Samples & Pos. Samples & Training & Eval. & Transfer Eval. \\
\hline Synthetic & 4081 & $\checkmark$ & $\checkmark$ & $\checkmark$ & \\
Natural (Negative) & 155809 & & $\checkmark$ & $\checkmark$ & \\
Natural (Defective) & 6586 & $\checkmark$ & & & $\checkmark$ \\
\hline
\end{tabular}

evaluating the fitted model's ability to transfer to unseen natural code.

These categories are further described in the subsections below. Note that these datasets require some special handling, which is discussed in section 5.1.4.

\subsubsection{Synthetic Projects}

The synthetic category consists of Juliet [60], a test suite for evaluating static analyzers. It consists of several thousand test cases, the majority of which are synthetic variants of some base defect, and covers 74 Common Weakness Enumerations (CWEs).

Synthetic test cases are derived from examples using a template system and allows for the systematic evaluation of an analyzer's ability to handle several variations of a defect. These variants can be classified into four categories: Base cases, control-flow dependent variants, intra-subroutine data-flow dependant variants, and interprocedural variants. Each subsequent variant increases the difficulty of the task. The first three generally only require reasoning about the contents of a single subroutine, ${ }^{1}$ while the last extends the problem across several subroutines and requires interprocedural analysis (IPA) to solve precisely.

A specific subset of CWEs offered by Juliet is used for these experiments, a listing of which can be found in table 5.2. All variants were used, including the interprocedural data-flow variants.

The difficulty of identifying a specific partial subgraph depends in part on the size of the partial subgraph in question. Statistics regarding the radii of various classes of subgraphs within the dataset is available in table 5.3. A typical positive label induced subgraph consists

\footnotetext{
${ }^{1}$ Some control flow variants do require reasoning about possible return values.
} 
Table 5.2: Population statistics for the utilized subset of Juliet. A sample consists of a single subroutine, and is considered positive if, and only if, it contains a vertex with a positive label.

\begin{tabular}{|c|c|c|c|c|c|c|c|}
\hline \multirow[b]{2}{*}{ Common Weakness Enumeration } & \multirow[b]{2}{*}{ Kind } & \multicolumn{3}{|c|}{ Per Sample } & \multicolumn{3}{|c|}{ Per Vertex } \\
\hline & & Count & $\%$ Tot. & $\%$ Pos. & Count & $\%$ Tot. & $\%$ Pos. \\
\hline \multirow{2}{*}{ HARD_CODE_KEY } & Original & 261 & 0.49 & 18.39 & 3370 & 0.16 & 7.29 \\
\hline & Simplified & 65 & 1.59 & 43.07 & 1746 & 0.37 & 7.96 \\
\hline \multirow{2}{*}{ HTTP_RESPONSE_SPLITTING } & Original & 12012 & 22.82 & 21.67 & 319554 & 15.23 & 2.84 \\
\hline & Simplified & 926 & 22.69 & 46.43 & 67176 & 14.39 & 3.02 \\
\hline \multirow{2}{*}{ LDAP_INJECTION } & Original & 3348 & 6.36 & 25.53 & 133958 & 6.38 & 4.52 \\
\hline & Simplified & 176 & 4.31 & 78.40 & 22750 & 4.87 & 5.48 \\
\hline \multirow{2}{*}{ SQL_INJECTION_JDBC } & Original & 21250 & 40.38 & 23.81 & 1262116 & 60.18 & 2.40 \\
\hline & Simplified & 1788 & 43.81 & 37.47 & 282164 & 60.48 & 2.10 \\
\hline \multirow{2}{*}{ UNVALIDATED_REDIRECT } & Original & 2349 & 4.46 & 22.09 & 45957 & 2.19 & 1.76 \\
\hline & Simplified & 118 & 2.89 & 81.35 & 8325 & 1.78 & 2.34 \\
\hline \multirow{2}{*}{ XPATH_INJECTION } & Original & 4004 & 7.60 & 21.67 & 150110 & 7.15 & 4.40 \\
\hline & Simplified & 404 & 9.89 & 33.16 & 41472 & 8.88 & 3.90 \\
\hline \multirow{2}{*}{ XSS } & Original & 9396 & 17.85 & 18.86 & 181920 & 8.67 & 7.35 \\
\hline & Simplified & 604 & 14.80 & 69.53 & 42869 & 9.18 & 7.77 \\
\hline \multirow{2}{*}{ Total } & Original & 52620 & 100.0 & 22.28 & 2096985 & 100.0 & 3.17 \\
\hline & Simplified & 4081 & 100.0 & 46.94 & 466502 & 100.0 & 3.11 \\
\hline
\end{tabular}

Table 5.3: Subgraph radii for the connected components within the Juliet dataset.

\begin{tabular}{rr}
\hline Connected Component & \multicolumn{1}{c}{ Radius } \\
\hline Non-singleton & $6.3 \pm 11.8$ \\
Pos. label connected & $51.4 \pm 29.0$ \\
Pos. label adjacent & $9.3 \pm 5.8$ \\
Pos. label induced & $6.7 \pm 5.2$ \\
\hline
\end{tabular}

roughly of a fifth of its connected component $(\mu=0.25, \sigma=0.24)$. Note that a sample may contain multiple connected components.

\subsubsection{Labelling}

The intended usage for Juliet is to evaluate an analyzer against each test case, where a given test case may span several subroutines or files. As such, it had to be adapted to fit the finer grain labelling scheme.

Special code comments in juliet were exploited to produce a dataset with per-vertex labels. These special comments annotate locations where a defect could manifest or where a defect mitigation occurs. Defects and mitigations are marked by code comments beginning with 
the string FLAW or POTENTIAL FLAW, and FIX, respectively. Examples for both are shown in listing 5.1. Note that defect annotation comments are pessimistic and exist even if defect mitigation occurs within the same subroutine.

Algorithm 1 describes the algorithm used to extract labels from Juliet annotations for a single subroutine. The only notable complication has to do with a control flow test variant where a mitigation path exists but is triggered unpredictably. The mitigation in this test case variant is ignored as it is unreliable (line 4 of algorithm 1 ). Note that vertices may appear on multiple (non-contiguous) lines, e.g. subroutine parameters.

No interprocedural propagation of annotations was performed. As a result, samples derived from interprocedural variants were pessimistically labelled and thus imply that all subroutine parameters are potentially tainted.

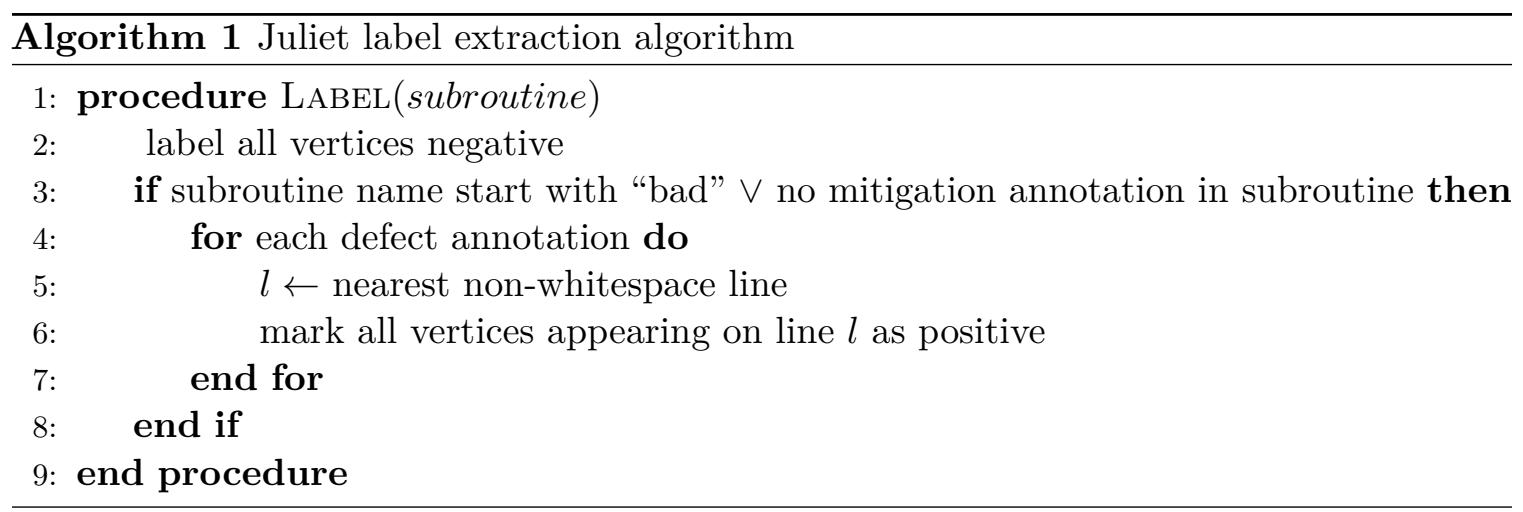

\subsubsection{Natural-Negative Projects}

The natural-negative category of projects consists of those which contain no defects of the kind modelled in Juliet. These served both as examples of natural code during training, contrasting with the synthetic nature of Juliet, and as a baseline for evaluating false positive rate of the fitted model. A complete listing of projects appearing in this category is provided in table B.1. 


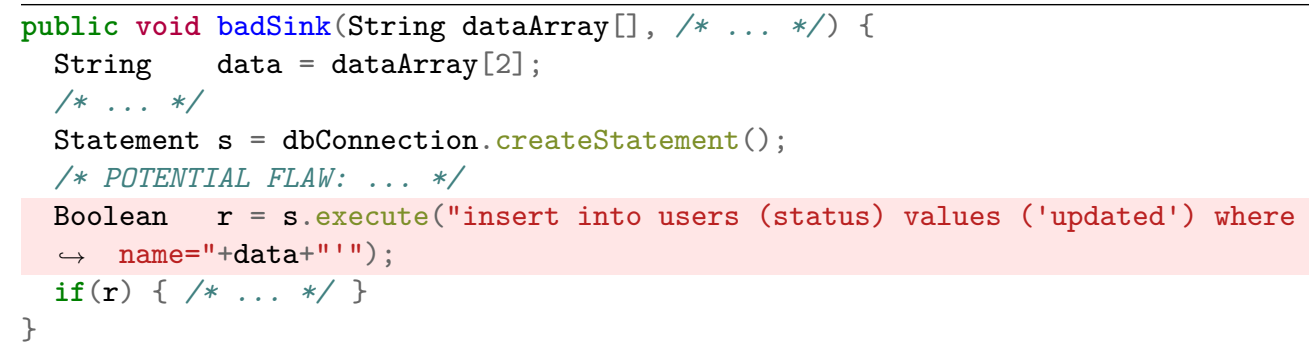

(a) Reduced example from test case (CWE89, execute, variant 66). Lines annotated as defective are highlighted in red. Note that any mitigation annotations in this subroutine would be ignored since the subroutine's name begins with "bad".

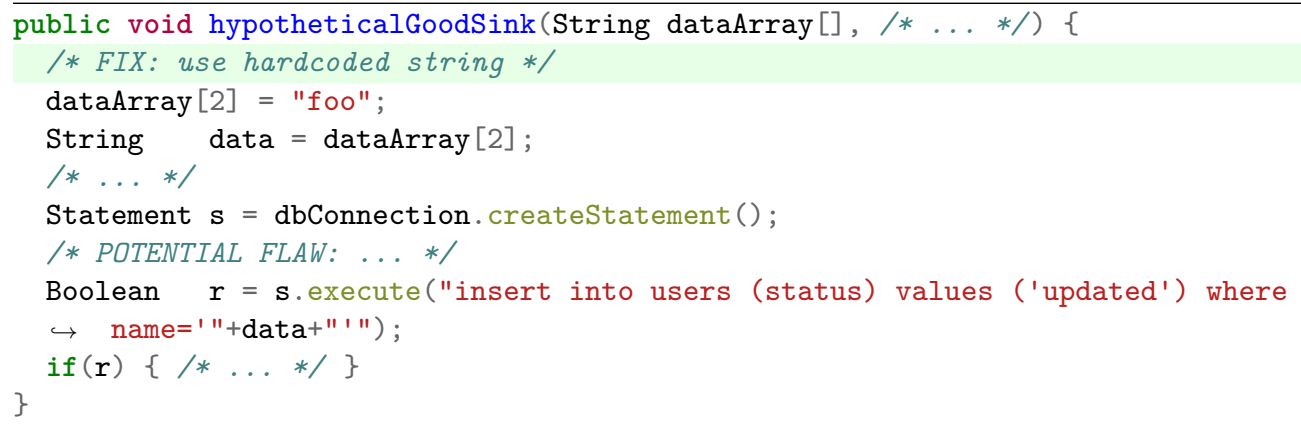

(b) Hypothetical fixed variant of a. The mitigation annotation is highlighted in green, and the defect annotation is ignored.

Listing 5.1: Reduced example of a Juliet test case. 
Table 5.4: Population statistics for natural-defective projects.

\begin{tabular}{rrrrrrrr}
\hline & \multicolumn{3}{c}{ Samples } & & \multicolumn{2}{c}{ Vertices } \\
\cline { 2 - 3 } \cline { 7 - 7 } Project & Count & \% Tot. & \% Pos. & & Count & \% Tot. \\
\hline Facebroke & 469 & 7.12 & Unk. & & 18805 & 4.78 \\
JavaVulnerableLabs & 470 & 7.13 & Unk. & 22027 & 5.60 \\
OWASP Benchmark* & 2740 & 41.60 & $51.6 \%$ & & 280843 & 71.47 \\
STONESOUP-INJ-1 & 1837 & 27.89 & Unk. & 56830 & 14.46 \\
Vuln-WebApps-Piggybank & 38 & 0.57 & Unk. & 355 & 0.09 \\
WebGOAT & 1032 & 15.66 & Unk. & 14054 & 3.57 \\
\hline Total & 6586 & 100.0 & Unk. & 392914 & 100.0 \\
\hline
\end{tabular}

* All subroutines from a given test case are merged into a single sample.

\subsubsection{Natural-Defective Projects}

The natural-defective category of projects consists of those which deliberately contain defects. These defects span several CWEs, including ones not present within the synthetic set. These projects were used to evaluate the ability of the model to transfer to natural code. A complete listing of projects appearing in this category is provided in table 5.4.

Most of these projects do not contain negative counterparts to positive samples, nor have they annotations or labels of any kind, making them unsuitable for inclusion in training set given a purely supervised approach. The OWASP Benchmark project is a partial exception as it posses both a mix of positive and negative test cases for various CWEs, in addition to sample-level labels. However, the OWASP Benchmark also makes extensive use of test cases spanning multiple subroutines, in addition to storing tainted and untainted data within the same data structures. ${ }^{2}$ The former is of particular note as it runs counter to the simplifying intra-subroutine-scope assumption and the pessimistic labelling specified for Juliet in section 5.1.1. This project should therefore be considered a particularly challenging unseen dataset.

\footnotetext{
${ }^{2}$ I have doubts regarding the representativeness of test cases mixing tainted and untainted information in data structures solely to complicate analysis. e. g. test cases which require reasoning about key-value pairs within the same map structure. These seem somewhat unrealistic.
} 


\subsubsection{Dataset Extraction \& Handling}

These datasets have a number of quirks which merit mention. These are enumerated below, along with any applied mitigations. All of these issues are related to various kinds of data imbalance.

\subsubsection{Vertex-Label Imbalance}

The most obvious imbalance is ratio of vertex-level class labels, with only $3.11 \%$ of vertices labelled as positive (defective).

The usual approaches for dealing with unbalanced classes involve either weighing the objective function such that expected loss for each class is equal, or altering the proportions of each class within the training set such that the classes are no longer imbalanced. The latter can be accomplished by resampling the minority classes or subsampling the majority classes.

However, none of these standard approaches are clearly applicable to this dataset. Subsampling and resampling can only be done at the sample level, which is already relatively balanced (46.94\% of Juliet samples are positive). Weighing the objective function is also undesirable as each class has sufficient distinct feature distributions (see below, section 5.1.4.3) to roughly discriminate between them. This can be show by noting the vertex level receiver operating characteristics (ROC) area under curve (AUC) of the $k=0$ model is near perfect (0.98, see table 5.7).

Given the above, no changes were applied to the dataset nor the objective function. However, care was taken to ensure all partitioning is done using stratified sampling based upon both class and CWE (see below).

\subsubsection{Test Case Imbalance}

The number of samples and, indirectly, test cases, for a given CWE varies wildly in Juliet ( $\mu=583, \sigma=613)$. Subsampling is not feasible, due to some CWEs having very few samples, e.g. HARD_CODE_KEY, and super-sampling would result in grossly exaggerated importance 
on the smallest minority classes. Loss reweighing is, again, unhelpful as the active symbol vocabularies can vary significantly between CWEs.

This is partially mitigated by using stratified sampling when partitioning the datasets.

\subsubsection{Symbol Vocabulary}

A common approach for modelling a large vocabulary of symbols with a non-uniform usage distribution is keep the most frequently occurring symbols and substitute the remainder with a single out-of-vocabulary (OOV) marker. This approach is not well suited for our task, as a given defect may require the presence of some specific, but rarely-occurring, symbol.

On the other hand, using a vocabulary consisting of every symbol encountered during training greatly increases the likelihood of learning a spurious relationship relating an incidental symbol and a defect. Such spurious relations are not limited to infrequently occurring symbols. An example of this can be seen in listing 5.2. A related problem concerns extremely rare symbols, whose occurrence can be used to trivially memorize the training set. A prototypical example of this is show in listing 5.3.

One solution is to select the vocabulary based on the intersection of symbols appearing both in Juliet and in either the natural-negative dataset or the standard Java libraries:

$$
\mathbb{S}_{\text {Active }}=\mathbb{S}_{\text {Juliet }} \cap\left(\mathbb{S}_{\text {NatNeg }} \cup \mathbb{S}_{\text {Java }}\right)
$$

Where $\mathbb{S}_{\text {Juliet }}, \mathbb{S}_{\mathrm{NatNeg}}$ and $\mathbb{S}_{\text {Java }}$ are the vocabularies used by Juliet, the natural-negative projects, and the Java standard libraries, respectively.

This avoids introducing symbols which appear only in negative contexts, eliminates incidental symbols unique to Juliet, and preserves rarely occurring, but non Juliet specific, symbols.

\subsubsection{Duplicate Samples}

Subroutine duplicates are not uncommon within most programs. These typically arise either due to inlined copies of a library, copy and pasted code, or as artefacts of the compiler's 


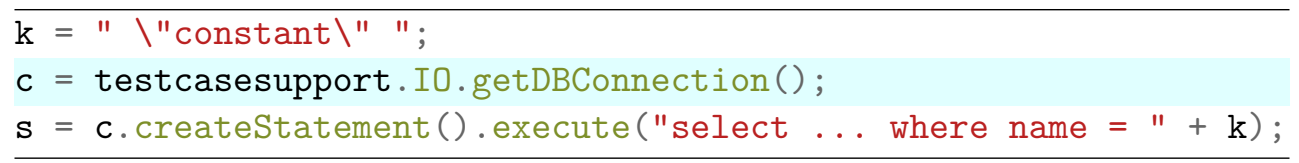

Listing 5.2: The symbol testcasesupport. IO.getDBConnection is relatively common within Juliet but never appears in other projects, allowing one to infer which project produced this sample. Note that the symbol SqlStatement: : execute, used on line 3 , occurs less frequent than testcasesupport. I0.getDBConnection within the Juliet corpus.

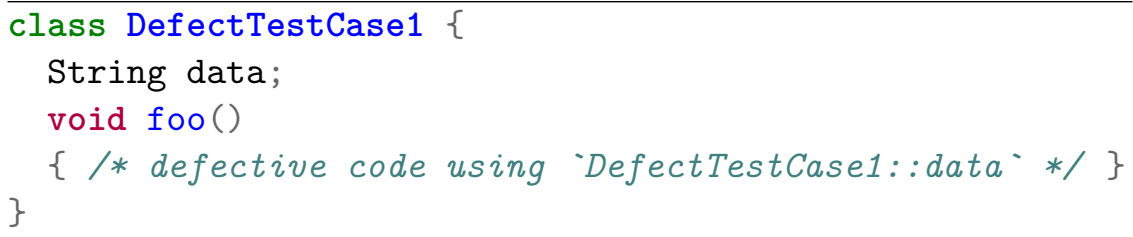

Listing 5.3: If the symbol DefectTestCase $1:$ : data is extremely rare, one can effectively memorize DefectTestCase1: : foo based upon the symbol's presence. The same issue can also occur through the type attribute of the implicit parameter this. Both cases require the defect subgraph to be connected to rare-symbol vertex, or that the model performs some sort of sample aggregation, e.g. continuous bag of words.

code generation. Roughly $3 \%$ to $5 \%$ of samples drawn from the projects were found to be duplicates and were discarded to avoid biasing the evaluation. Duplicates were identified by keying on the SHAKE-256 of their decompiled source code representation using fully qualified types. This deduplication pass served mainly as a quick a filter for removing trivial duplicates from the dataset.

A second deduplication pass was performed after graph simplification (section 4.1.2) and vocabulary handling (section 5.1.4.3), as both of these processes can cause previously distinct graphs to become equivalent. In this second pass, duplicates were identified and discarded using a graph isomorphism test [20]. Samples in the second pass were considered duplicates if, and only if, their graphs were isomorphic and they had identical labels. The consideration of the labels is necessary as many of the applied transformations are not injective. A single instance is kept for each distinct graph and label pair. 
Table 5.5: Initializers for model parameters.

\begin{tabular}{rr}
\hline Parameter Type & Initialization \\
\hline Symbol Embeddings & Uniform Random $\in[-1,1]$ \\
Recurrence Parameters ${ }^{3}$ & Identity Matrix [52] \\
Others & Glorot Uniform [29] \\
\hline
\end{tabular}

\subsection{Training Protocol}

All models, including baseline variants, were fitted using the same protocol. Parameters are initialized as per table 5.5 and optimized using RMSProp [83] without momentum. Training continued until the average vertex cross-entropy on the development set failed to decrease by at least $0.5 \%$ over 10 epochs, or 500 epochs elapsed. Samples were aggregated into mini-batches of $15 \mathrm{k}$ vertices $(\approx 575$ samples $)$ and shuffled each epoch.

\subsubsection{Hyper-parameters}

Hyper-parameters not specified in chapter 4 were selected by a grid-search using the training and development subsets. All hyper-parameter values, including those chosen manually, are enumerated in table 5.6.

Hyper-parameters were chosen in groups, designated by the partitioning in table 5.6, in order to limit the overall search domain. For example, while selecting regularization and network width, the learning rate was held at $10^{-4}$. Parameters were chosen based on lowest cross-entropy on the development set after twenty epochs.

\subsection{Primary Results}

The proposed model is evaluated using neighbourhood sizes $k \in[0,10]$. In addition to these, a continuous bag of words (CBOW) like model variant is also evaluated, where the $N^{k}(v)$

\footnotetext{
${ }^{3}$ Recurrence parameters are only used by the classic GRU variant in Appendix A. The proposed model has a recurrence connection, but does not (directly) project it.
} 
Table 5.6: List of hyper-parameters. This table also include hyper-parameters which are chosen manually. Parameters sharing the a common background colour were selected jointly using a grid search over their listed domains.

\begin{tabular}{rrr}
\hline Hyper-parameter & Value & Domain \\
\hline Learning Rate & $10^{-4}$ & $10^{[-5,-3]}$ \\
Supertype Drop Rate & 0.2 & $0,0.05,0.1,0.2,0.3$ \\
L2 Regularization $(\lambda)$ & $10^{-1}$ & $0,10^{[-3,0]}$ \\
$N^{k}$ Embedding Width $\left(\eta_{G}\right)$ & 128 & $2^{[5,7]}$ \\
Symbol Embedding Width $\left(\eta_{E}\right)$ & 32 & $2^{[4,6]}$ \\
\hline Max Const. Val. Length & $2^{7}$ & Manual \\
Max Operand Index & 16 & Manual \\
Batch Size & 15k Vertices & Manual \\
RMSProp Decay & 0.9 & Manual \\
\hline
\end{tabular}

embedding $\mathbf{h}_{v}^{k}$ was replaced with:

$$
h_{v}^{\mathrm{CBOW}}=\tau\left(\mathbf{W} \sum_{v \in V} X_{v}+|V| \mathbf{b}\right)
$$

The $k=0$ and CBOW models serve as baselines. Neither made use of dependence features nor the graphs' connectivity structure. Each experiment used a 5-fold evaluation with different random initialization for each fold, and the variance between folds reported. Each model used the same k-fold splits.

The results are tabulated in table 5.7 and fig. 5.1, and a breakdown of the $k=10$ model per CWE and test case variant is provided in table 5.8. Figure 5.2 compares the models' performance against FindSecBugs [10], an open source static analyzer for security defects. Unlike the proposed models, FindSecBugs makes use of interprocedural analyses, and often reports incidental defects or suggestions. In order to make a fair(er) comparison between FindSecBugs and the proposed approach, the following scoring protocol was used: A FindSecBugs prediction was considered correct if reported the correct $\mathrm{CWE}^{4}$ for the test case, and the report mentioned either the vulnerable sink method ("bad sink") or the entry point for the test case (the "bad method"). If no predictions involved a given test case's "bad method", then it was considered a false negative.

\footnotetext{
${ }^{4}$ This need not be an exact match; there exists some overlap between CWEs. e. g. CWE 79, 80, 81, and 83 are all variants on XSS.
} 
Except for $k=0$ baseline, all models achieved excellent performances in terms of ROC, average precision (AP), and $\phi$ Coefficient. Performance generally improved as $k$ increases, with rapid gains up to $k=4$ followed by diminishing returns past $k=6$. This infliction point is likely related to the typical radius for a non-singleton connected component or label-induced subgraph, see table 5.3 and the no-vertex-feature $k=5$ variant in section 5.3.1.

The CBOW baseline proved to be a strong baseline despite having no access to the connectivity of the graph or edge features. It outperformed other models for most samplelevel metrics, and was competitive on vertex-level metrics against models up to $k=4$. However, it completely failed to transfer to unseen projects (see section 5.4).

\subsubsection{Feature-set Ablation}

As mentioned in section 4.1.3, while each feature does provide some information which cannot be inferred from others, none are completely independent: A member reference imparts an upper-bound on the result type of the operation. Similarly, a constant value affects the type due to the small number of constant value types, and the instruction determines whether a member or constant value is used.

In order to evaluate the utility of each operation feature for the primary dataset, a series of experiments was conducted using the CBOW, $k=0$, and $k=5$ models. Given the small number of operation features and their interdependencies this can be achieved with reasonable overhead by producing model variants fitted using various subsets of the vertex features. Edge features were not ablated in this experiment.

The results are tabulated in table 5.9. Note that the metrics reported in table 5.9 exclude samples from natural-negative projects. This was done to avoid saturating metrics sensitive to the relative size of the negative class. An equivalent listing considering all samples is provided in table D.1.

The results are generally as one would expect, with the member feature proving to be the most informative feature for all models, followed by the type or constant value, and the instruction as the least informative feature for this dataset. While baseline models are 


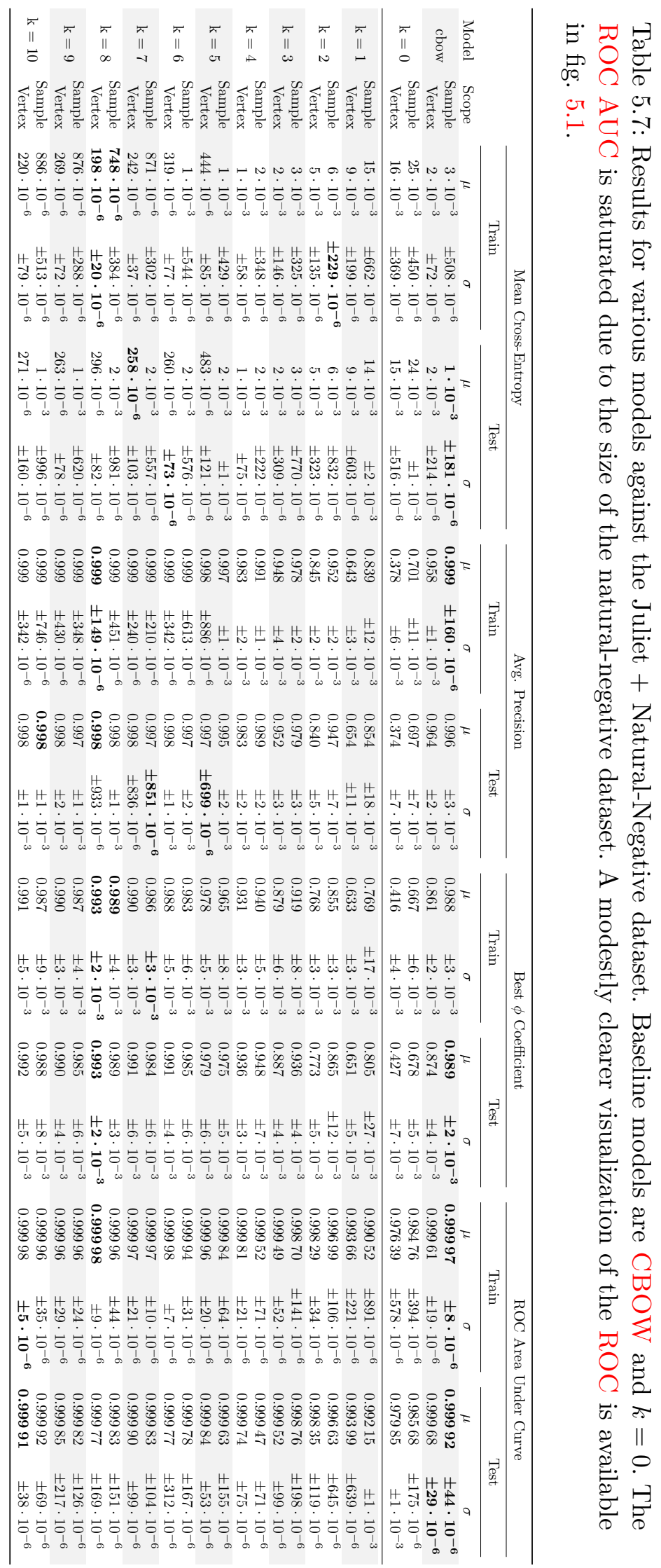



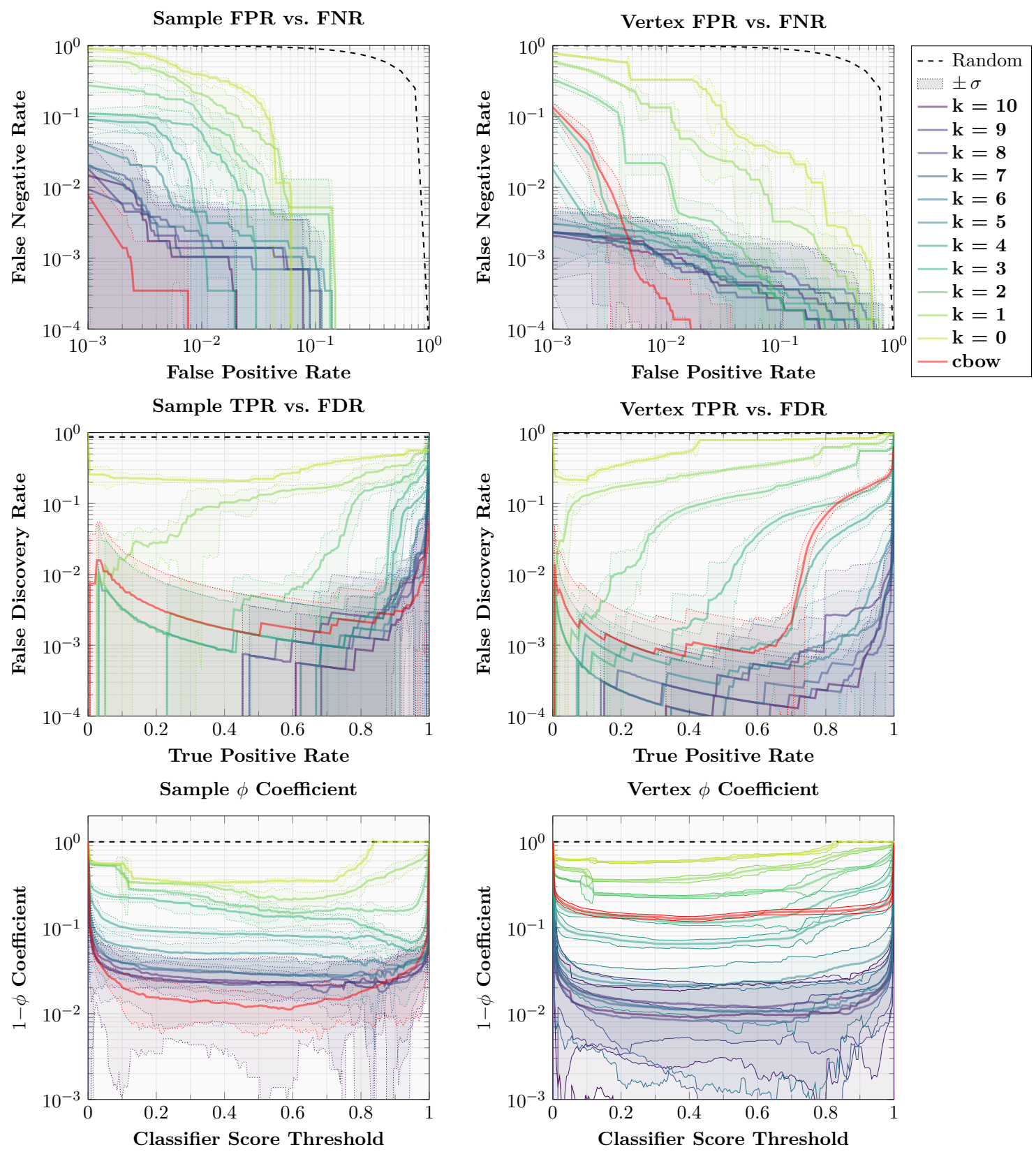

Fig. 5.1: Test set performance metrics for Juliet and natural-negative projects. These plots correspond to the metrics reported in table 5.7 but use the complementing metric for the y-axis and log scaling. This is necessary for legibility as the original y-axis values are saturated near 1 for most models. Note the use of log-log axes for false discovery rate (FDR) vs. false positive rate (FPR). 
Table 5.8: Juliet performance metrics using a $k=10$ model, decomposed per (CWE, variant) pair.

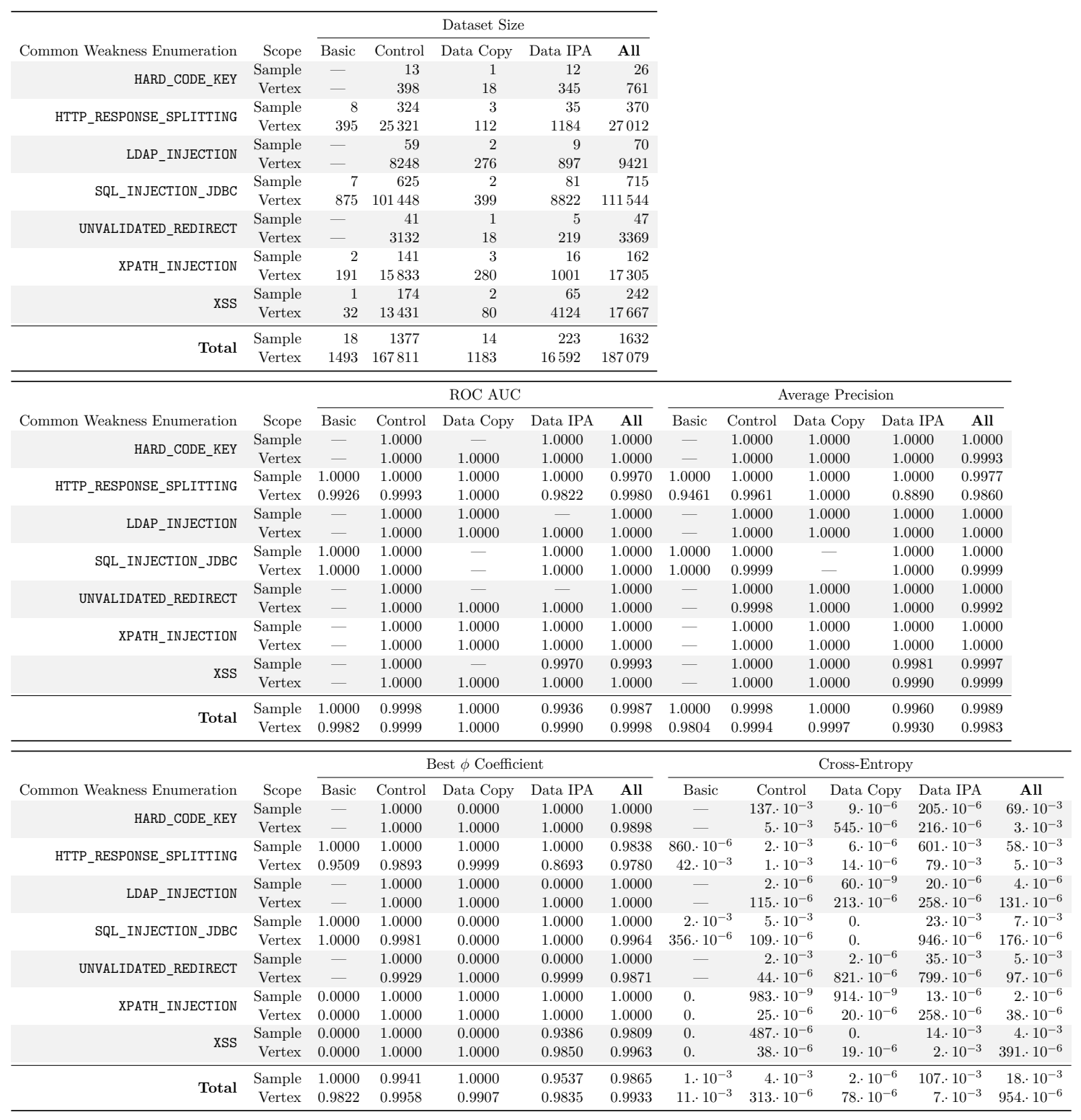



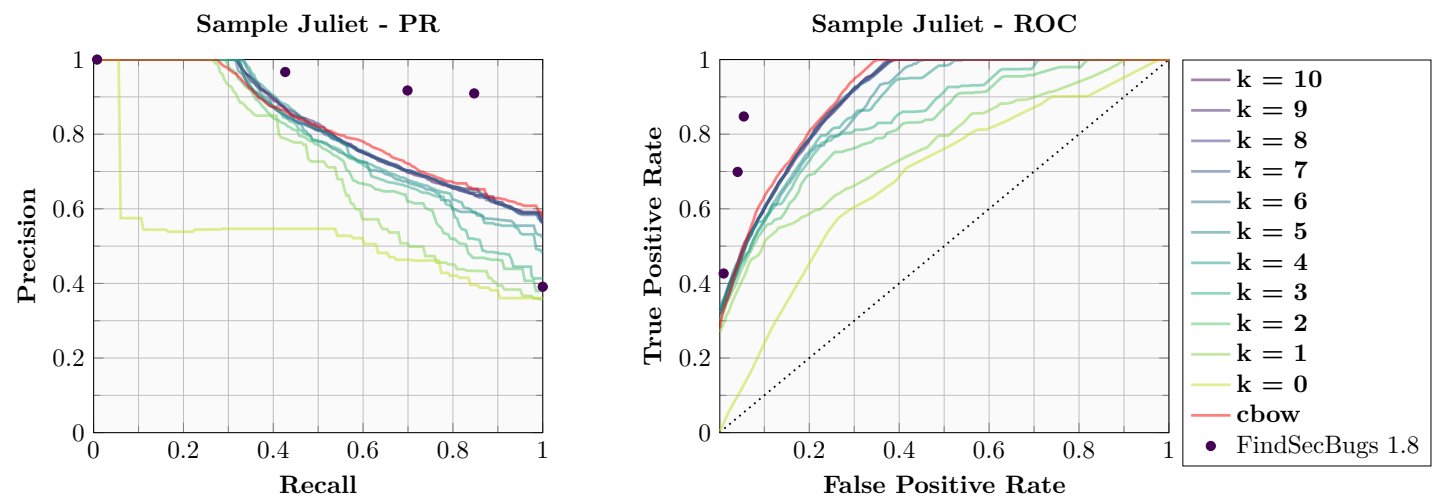

Fig. 5.2: ROC and AP for the Juliet subset of the primary dataset. Performance values for FindSecBugs [10] at different confidence thresholds are marked for comparison.

limited to using vertex features, the no-vertex-features CBOW baseline is able to exceed random-guessing using the sample's vertex count via its bias' coefficient. What was surprising was the unexpected informativeness of the edge features and the graph structure itself, as evidenced by the $k=5$ model achieving a best $\phi$ Coefficient of 0.76 and 0.83 per sample and vertex, respectively.

\subsection{Transferring to Unseen Projects}

The fitted models were applied to the natural-defective dataset (see section 5.1.3) in order to evaluate their ability to transfer from synthetic defect examples to unseen natural defects. The sample-level $\operatorname{ATP}(0.2)$ and ROC was measured for all natural-defective projects, as well as the $\phi$ Coefficient for labelled projects. Positive samples for CWEs not present within the training set were excluded as these would affect the $\phi$ Coefficient. Labels were provided for unlabelled samples which received a prediction $\geq 0.2$ by at least one model, encompassing roughly $10 \%$ of the unlabelled dataset.

The models used in this experiment were fitted against the entire primary dataset, except for the development subset $(\approx 10 \%)$, using the same protocol as the primary evaluation. The results are tabulated in table 5.10, and fig. 5.3. The ROC plot in fig. 5.3 includes reported performance values for three open source static analyzers for comparison [90], each of which make use of interprocedural analyses. 
Table 5.9: Feature-set ablation evaluation for primary dataset, using baseline and $k=5$ models. Of note is the surprising effectiveness of the no-vertex-features variant of the $k=5$ model (highlighted in light blue).

\begin{tabular}{|c|c|c|c|c|c|c|c|c|c|c|c|c|c|}
\hline \multirow{3}{*}{$\begin{array}{r}\text { Model } \\
\text { cbow }\end{array}$} & \multirow{3}{*}{$\begin{array}{c}\text { Scope } \\
\text { Sample }\end{array}$} & \multicolumn{4}{|c|}{ Features } & \multicolumn{2}{|c|}{ Cross-Entropy } & \multicolumn{2}{|c|}{ Avg. Precision } & \multicolumn{2}{|c|}{ Best $\phi$ Coeff. } & \multicolumn{2}{|c|}{ ROC AUC } \\
\hline & & Instr. & Type & Member & Const. & Train & Test & Train & Test & Train & Test & Train & Test \\
\hline & & & & & & 2 & 2 & 0.481 & 0.480 & 0.085 & 0.078 & 0.505 & 0.524 \\
\hline & & & & & $\checkmark$ & $428 \cdot 10^{-3}$ & $402 \cdot 10^{-3}$ & 0.974 & 0.976 & 0.838 & 0.848 & 0.977 & 0.979 \\
\hline & & & & $d$ & & $149 \cdot 10^{-3}$ & $92 \cdot 10^{-3}$ & 0.938 & 0.960 & 0.815 & 0.821 & 0.960 & 0.970 \\
\hline & & & $\checkmark$ & & & $476 \cdot 10^{-3}$ & $475 \cdot 10^{-3}$ & 0.780 & 0.768 & 0.523 & 0.526 & 0.828 & 0.829 \\
\hline & & $\checkmark$ & & & & $618 \cdot 10^{-3}$ & $630 \cdot 10^{-3}$ & 0.828 & 0.814 & 0.672 & 0.661 & 0.889 & 0.877 \\
\hline & & & & $d$ & $d$ & $80 \cdot 10^{-3}$ & $80 \cdot 10^{-3}$ & 1.000 & 1.000 & 0.983 & 0.982 & 1.000 & 1.000 \\
\hline & & & $\checkmark$ & $\checkmark$ & & $88 \cdot 10^{-3}$ & $80 \cdot 10^{-3}$ & 0.973 & 0.975 & 0.877 & 0.881 & 0.980 & 0.982 \\
\hline & & $\checkmark$ & & 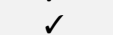 & & $87 \cdot 10^{-3}$ & $77 \cdot 10^{-3}$ & 0.980 & 0.961 & 0.867 & 0.852 & 0.984 & 0.976 \\
\hline & & & $\checkmark$ & 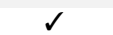 & $d$ & $18 \cdot 10^{-3}$ & $18 \cdot 10^{-3}$ & 1.000 & 1.000 & 0.991 & 0.995 & 1.000 & 1.000 \\
\hline & & $d$ & $\checkmark$ & $\checkmark$ & & $39 \cdot 10^{-3}$ & $32 \cdot 10^{-3}$ & 0.990 & 0.991 & 0.929 & 0.931 & 0.992 & 0.993 \\
\hline & & $\checkmark$ & $\checkmark$ & $\checkmark$ & $\checkmark$ & $25 \cdot 10^{-3}$ & $8 \cdot 10^{-3}$ & 0.999 & 1.000 & 0.976 & 0.989 & 0.999 & 1.000 \\
\hline & Vertex & & & & & $140 \cdot 10^{-3}$ & $136 \cdot 10^{-3}$ & 0.030 & 0.029 & 0.035 & 0.031 & 0.468 & 0.479 \\
\hline & & & & & $\checkmark$ & $66 \cdot 10^{-3}$ & $68 \cdot 10^{-3}$ & 0.418 & 0.407 & 0.420 & 0.421 & 0.909 & 0.903 \\
\hline & & & & $\checkmark$ & & $39 \cdot 10^{-3}$ & $41 \cdot 10^{-3}$ & 0.655 & 0.664 & 0.618 & 0.623 & 0.957 & 0.955 \\
\hline & & & $d$ & & & $54 \cdot 10^{-3}$ & $54 \cdot 10^{-3}$ & 0.409 & 0.425 & 0.417 & 0.433 & 0.931 & 0.936 \\
\hline & & $d$ & & & & $61 \cdot 10^{-3}$ & $60 \cdot 10^{-3}$ & 0.328 & 0.339 & 0.351 & 0.361 & 0.938 & 0.940 \\
\hline & & & & $\checkmark$ & $\checkmark$ & $20 \cdot 10^{-3}$ & $20 \cdot 10^{-3}$ & 0.896 & 0.893 & 0.825 & 0.819 & 0.990 & 0.990 \\
\hline & & & $\checkmark$ & $\checkmark$ & & $27 \cdot 10^{-3}$ & $27 \cdot 10^{-3}$ & 0.797 & 0.801 & 0.691 & 0.690 & 0.989 & 0.989 \\
\hline & & $d$ & & $\checkmark$ & & $22 \cdot 10^{-3}$ & $22 \cdot 10^{-3}$ & 0.801 & 0.784 & 0.685 & 0.673 & 0.989 & 0.989 \\
\hline & & & $\checkmark$ & $\checkmark$ & $\checkmark$ & $8 \cdot 10^{-3}$ & $8 \cdot 10^{-3}$ & 0.959 & 0.961 & 0.863 & 0.866 & 0.998 & 0.999 \\
\hline & & $d$ & 2 & $d$ & & $17 \cdot 10^{-3}$ & $18 \cdot 10^{-3}$ & 0.840 & 0.839 & 0.726 & 0.727 & 0.992 & 0.991 \\
\hline & & $\checkmark$ & $\checkmark$ & $\checkmark$ & $\checkmark$ & $8 \cdot 10^{-3}$ & $7 \cdot 10^{-3}$ & 0.963 & 0.969 & 0.869 & 0.880 & 0.999 & 0.999 \\
\hline $\mathrm{k}=0$ & Sample & & & & & 2 & 2 & 0.470 & 0.470 & 0.000 & 0.000 & 0.500 & 0.500 \\
\hline & & & & & $\checkmark$ & $486 \cdot 10^{-3}$ & $467 \cdot 10^{-3}$ & 0.707 & 0.719 & 0.455 & 0.474 & 0.751 & 0.776 \\
\hline & & & & $\checkmark$ & & $365 \cdot 10^{-3}$ & $392 \cdot 10^{-3}$ & 0.697 & 0.656 & 0.424 & 0.381 & 0.730 & 0.691 \\
\hline & & & $d$ & & & $934 \cdot 10^{-3}$ & $943 \cdot 10^{-3}$ & 0.560 & 0.559 & 0.253 & 0.249 & 0.562 & 0.558 \\
\hline & & 2 & & & & 2 & 2 & 0.470 & 0.469 & 0.019 & 0.000 & 0.500 & 0.500 \\
\hline & & & & $\checkmark$ & $\checkmark$ & $300 \cdot 10^{-3}$ & $300 \cdot 10^{-3}$ & 0.708 & 0.705 & 0.451 & 0.454 & 0.759 & 0.761 \\
\hline & & & 2 & $d$ & & $331 \cdot 10^{-3}$ & $345 \cdot 10^{-3}$ & 0.676 & 0.687 & 0.402 & 0.418 & 0.715 & 0.716 \\
\hline & & $\checkmark$ & & $\checkmark$ & & $312 \cdot 10^{-3}$ & $297 \cdot 10^{-3}$ & 0.673 & 0.699 & 0.398 & 0.429 & 0.706 & 0.739 \\
\hline & & & $\checkmark$ & $\checkmark$ & $\checkmark$ & $227 \cdot 10^{-3}$ & $238 \cdot 10^{-3}$ & 0.722 & 0.728 & 0.484 & 0.507 & 0.782 & 0.776 \\
\hline & & $\checkmark$ & $\checkmark$ & $\checkmark$ & & $381 \cdot 10^{-3}$ & $373 \cdot 10^{-3}$ & 0.675 & 0.684 & 0.402 & 0.412 & 0.712 & 0.721 \\
\hline & & $\checkmark$ & $\checkmark$ & $\checkmark$ & $\checkmark$ & $208 \cdot 10^{-3}$ & $215 \cdot 10^{-3}$ & 0.724 & 0.727 & 0.453 & 0.463 & 0.774 & 0.771 \\
\hline & Vertex & & & & & $149 \cdot 10^{-3}$ & $152 \cdot 10^{-3}$ & 0.031 & 0.032 & 0.000 & 0.000 & 0.500 & 0.500 \\
\hline & & & & & $\checkmark$ & $125 \cdot 10^{-3}$ & $126 \cdot 10^{-3}$ & 0.168 & 0.169 & 0.307 & 0.308 & 0.646 & 0.644 \\
\hline & & & & $\checkmark$ & & $105 \cdot 10^{-3}$ & $99 \cdot 10^{-3}$ & 0.290 & 0.268 & 0.419 & 0.398 & 0.842 & 0.839 \\
\hline & & & $\checkmark$ & & & $107 \cdot 10^{-3}$ & $108 \cdot 10^{-3}$ & 0.169 & 0.165 & 0.287 & 0.281 & 0.857 & 0.854 \\
\hline & & $d$ & & & & $120 \cdot 10^{-3}$ & $127 \cdot 10^{-3}$ & 0.064 & 0.067 & 0.191 & 0.195 & 0.769 & 0.768 \\
\hline & & & & $\checkmark$ & 2 & $77 \cdot 10^{-3}$ & $77 \cdot 10^{-3}$ & 0.436 & 0.438 & 0.510 & 0.510 & 0.928 & 0.929 \\
\hline & & & $d$ & 4 & & $77 \cdot 10^{-3}$ & $74 \cdot 10^{-3}$ & 0.337 & 0.331 & 0.438 & 0.437 & 0.940 & 0.940 \\
\hline & & $d$ & & 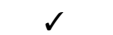 & & $78 \cdot 10^{-3}$ & $77 \cdot 10^{-3}$ & 0.315 & 0.322 & 0.411 & 0.417 & 0.927 & 0.926 \\
\hline & & & $\checkmark$ & $\checkmark$ & $\checkmark$ & $57 \cdot 10^{-3}$ & $56 \cdot 10^{-3}$ & 0.469 & 0.468 & 0.532 & 0.526 & 0.968 & 0.967 \\
\hline & & $\checkmark$ & $\checkmark$ & $\checkmark$ & & $78 \cdot 10^{-3}$ & $81 \cdot 10^{-3}$ & 0.335 & 0.345 & 0.437 & 0.446 & 0.938 & 0.939 \\
\hline & & $\checkmark$ & $\checkmark$ & $\checkmark$ & $\checkmark$ & $52 \cdot 10^{-3}$ & $51 \cdot 10^{-3}$ & 0.476 & 0.472 & 0.534 & 0.526 & 0.967 & 0.967 \\
\hline $\mathrm{k}=5$ & Sample & & & & & $156 \cdot 10^{-3}$ & $133 \cdot 10^{-3}$ & 0.919 & 0.927 & 0.763 & 0.756 & 0.932 & 0.936 \\
\hline & & & & & $\checkmark$ & $14 \cdot 10^{-3}$ & $15 \cdot 10^{-3}$ & 0.994 & 0.994 & 0.934 & 0.941 & 0.994 & 0.994 \\
\hline & & & & $\checkmark$ & & $28 \cdot 10^{-3}$ & $48 \cdot 10^{-3}$ & 0.980 & 0.974 & 0.880 & 0.867 & 0.983 & 0.979 \\
\hline & & & $d$ & & & $63 \cdot 10^{-3}$ & $51 \cdot 10^{-3}$ & 0.953 & 0.958 & 0.776 & 0.793 & 0.954 & 0.961 \\
\hline & & $\checkmark$ & & & & $50 \cdot 10^{-3}$ & $46 \cdot 10^{-3}$ & 0.977 & 0.980 & 0.836 & 0.852 & 0.977 & 0.981 \\
\hline & & & & $\checkmark$ & $\checkmark$ & $28 \cdot 10^{-3}$ & $31 \cdot 10^{-3}$ & 0.998 & 0.997 & 0.970 & 0.970 & 0.998 & 0.997 \\
\hline & & & $\checkmark$ & $d$ & & $32 \cdot 10^{-3}$ & $24 \cdot 10^{-3}$ & 0.983 & 0.983 & 0.891 & 0.884 & 0.986 & 0.986 \\
\hline & & $d$ & & 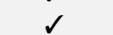 & & $23 \cdot 10^{-3}$ & $16 \cdot 10^{-3}$ & 0.996 & 0.997 & 0.955 & 0.964 & 0.996 & 0.997 \\
\hline & & & $d$ & 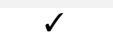 & $d$ & $10 \cdot 10^{-3}$ & $13 \cdot 10^{-3}$ & 0.999 & 0.998 & 0.979 & 0.968 & 0.998 & 0.997 \\
\hline & & $\checkmark$ & $\checkmark$ & $d$ & & $7 \cdot 10^{-3}$ & $9 \cdot 10^{-3}$ & 0.999 & 0.999 & 0.967 & 0.971 & 0.999 & 0.999 \\
\hline & & $\checkmark$ & $\checkmark$ & $\checkmark$ & $\checkmark$ & $39 \cdot 10^{-3}$ & $31 \cdot 10^{-3}$ & 0.997 & 0.996 & 0.962 & 0.961 & 0.997 & 0.996 \\
\hline & Vertex & & & & & $20 \cdot 10^{-3}$ & $20 \cdot 10^{-3}$ & 0.883 & 0.892 & 0.818 & 0.829 & 0.996 & 0.996 \\
\hline & & & & & $d$ & $3 \cdot 10^{-3}$ & $3 \cdot 10^{-3}$ & 0.986 & 0.987 & 0.922 & 0.927 & 0.999 & 1.000 \\
\hline & & & & $\checkmark$ & & $5 \cdot 10^{-3}$ & $5 \cdot 10^{-3}$ & 0.970 & 0.964 & 0.938 & 0.931 & 0.999 & 0.999 \\
\hline & & & $d$ & & & $10 \cdot 10^{-3}$ & $11 \cdot 10^{-3}$ & 0.918 & 0.923 & 0.846 & 0.850 & 0.997 & 0.996 \\
\hline & & $d$ & & & & $15 \cdot 10^{-3}$ & $15 \cdot 10^{-3}$ & 0.949 & 0.951 & 0.869 & 0.864 & 0.998 & 0.998 \\
\hline & & & & $\checkmark$ & $d$ & $3 \cdot 10^{-3}$ & $3 \cdot 10^{-3}$ & 0.998 & 0.998 & 0.987 & 0.987 & 1.000 & 1.000 \\
\hline & & & $d$ & 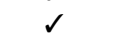 & & $3 \cdot 10^{-3}$ & $4 \cdot 10^{-3}$ & 0.973 & 0.972 & 0.933 & 0.928 & 0.999 & 0.999 \\
\hline & & $d$ & & $d$ & & $3 \cdot 10^{-3}$ & $3 \cdot 10^{-3}$ & 0.996 & 0.997 & 0.970 & 0.970 & 1.000 & 1.000 \\
\hline & & & $\checkmark$ & $\checkmark$ & $\checkmark$ & $1 \cdot 10^{-3}$ & $883 \cdot 10^{-6}$ & 0.998 & 0.999 & 0.988 & 0.988 & 1.000 & 1.000 \\
\hline & & $d$ & 4 & 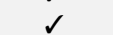 & & $2 \cdot 10^{-3}$ & $2 \cdot 10^{-3}$ & 0.998 & 0.997 & 0.979 & 0.982 & 1.000 & 1.000 \\
\hline & & $\checkmark$ & $\checkmark$ & $\checkmark$ & $\checkmark$ & $3 \cdot 10^{-3}$ & $3 \cdot 10^{-3}$ & 0.997 & 0.998 & 0.976 & 0.976 & 1.000 & 1.000 \\
\hline
\end{tabular}


All models struggled with this task, despite the abundance of defects within in dataset. Most models achieved an excellent ROC for the majority of projects, but did so by being exhibiting relatively high specificity and low sensitivity. The OWASP Benchmark project, however, was particularly difficult for all models. None achieved a notable ROC or AP for this project, and all exhibited an abysmal best $\phi$ coefficient. This was not unexpected, as this particular test suite focuses heavily on defect variants requiring interprocedural analysis. Interestingly, the most successful models for the natural-defective dataset were those with smaller neighbourhood upper bounds, e.g. $k \in[1,5]$. Models with larger effective neighbourhoods, including the CBOW baseline, struggle to maintain a reasonable ROC. 


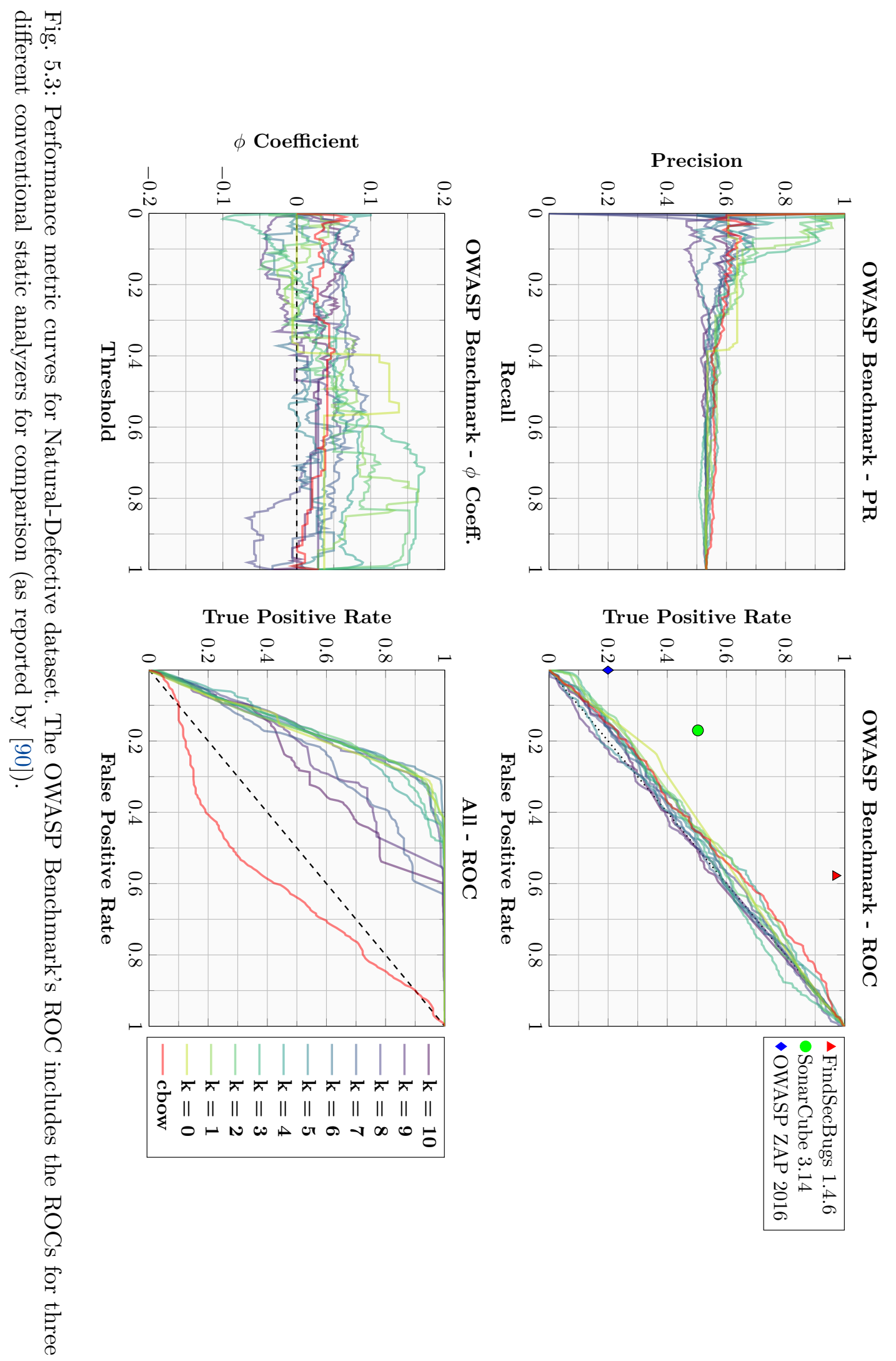




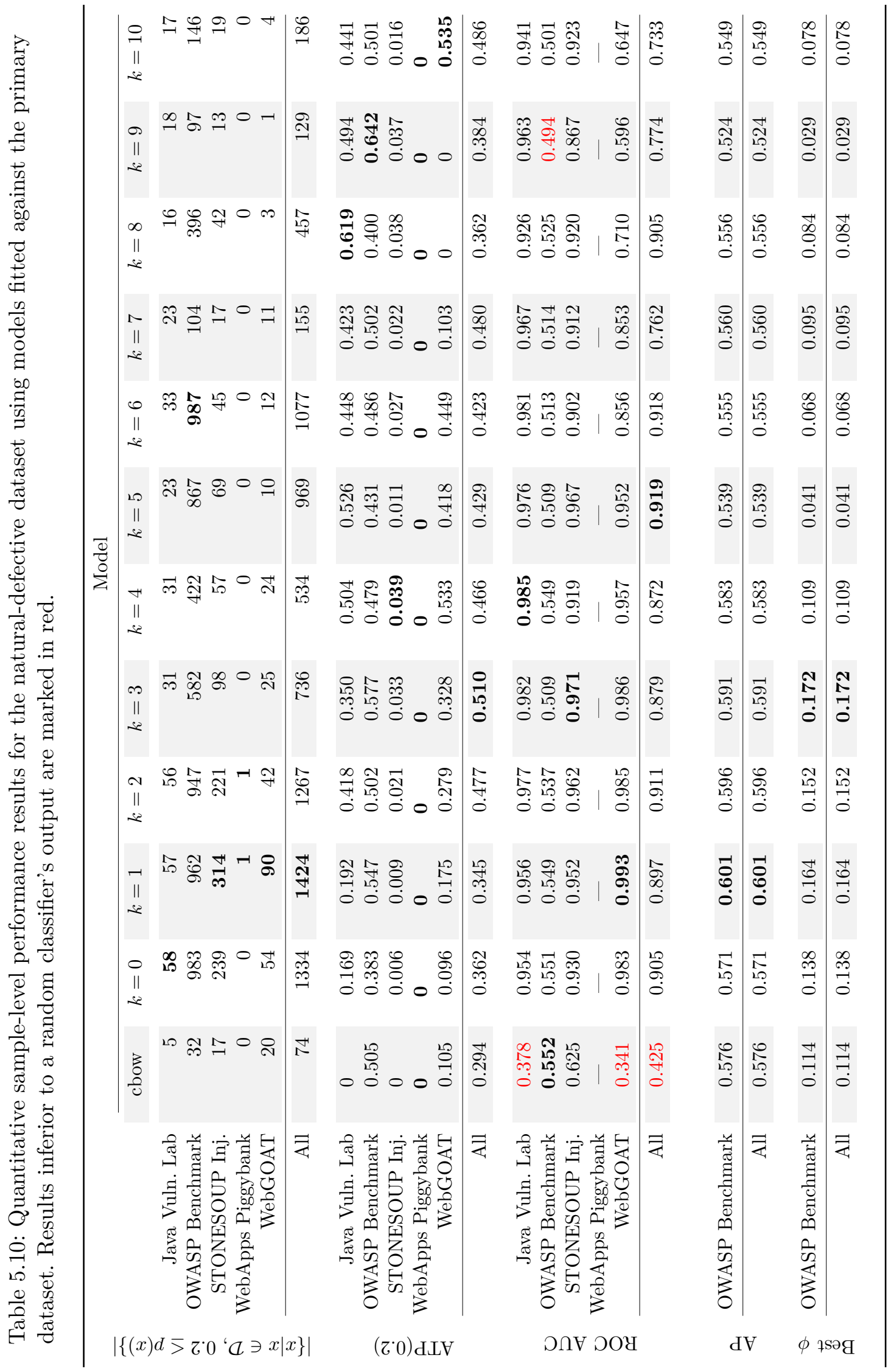




\section{Chapter 6}

\section{Conclusions}

I demonstrated that a neural graph classifier fitted against a mixture of natural negative code and synthetic test suites can achieve excellent precision and ROC. When applied to unseen natural code, the models mostly retained their ROC, but exhibited poor precision and recall. None of the model variants were able to transfer to the OWASP Benchmark suite.

\section{Summary of Contributions.}

I implemented a new approach for detecting software defects caused by incorrect operation dependencies based upon learning to identify partial subgraphs within a program's dependence graph. While whole graph classification is not a novel task in machine learning, to the best of my knowledge this combination of subgraph classification and application domain is novel.

I demonstrated that this approach can achieve excellent performance against an adapted subset of Juliet [60], and a very modest ability to transfer to unseen natural defective code. This approach even managed some minimal success against the OWASP Benchmark project, despite lacking any ability to perform interprocedural analyses due to simplifying assumptions.

\subsection{Future Work}

Interprocedural Analysis. In order to simplify the task, the scope of sample is limited to a single subroutine. This is a significant limitation as defects can easily span across several subroutines, if only via data-flow. One naive solution is to expand invocations up to some given depth by substituting invocations with their callee's dependence graph. However, this 
could dramatically increase the size of the graphs and would only be effective up to an expanded depth of $k$. A possible solution would be to infer which callees, including indirect callees, would be good candidates for inlining. Such an approach bears some similarities to MERLIN's inference of source-sink-sanitizer triplets [12].

Symbol Vocabulary. As discussed in section 5.1.4.3, proper handling of the symbol vocabulary is important. Some adjustments were made, given the unusual nature of the symbol vocabulary, but these adaptations were kept to a minimum. It behooves us to consider this issue in more detail, particularly with regards the handling of rare symbols. Possibilities include adapting the techniques presented in Herbelot and Baroni [38] for initializing the embedding of a new symbol based on the symbol's entity definition, e.g. using an embedding of a function as its initial representation, or its usage contexts.

Few Shot \& Active Learning. Requiring hundreds of examples to learn a handful of variants for a single defect is not practical, nor necessarily possible for all defects. An application-specific defect specific might only have a small handful of instances; it would be perverse to require the user to deliberately create more. Ideally, the user should only have to annotate one or two instances of a defect before receiving feedback. Users should also be able to refine the inferred specification by correcting erroneous predictions with additional labelling, if so desired.

A practical tool must therefore support few-shot labelling, as well as active learning. This could include incremental vocabulary expansion by initializing new symbols using the embeddings of known symbols [38]. It may be difficult to generalize any kind of transfer learning to entirely novel defects, but it should be possible to apply to defect families, e. g. defects involving string concatenation, and defect variants, e. g. Juliet's data and control flow variants. 


\section{ApPENDix A \\ Recurrence Connection Behaviour}

Laurent and von Brecht [51] demonstrated the presence of strange attractors in the recurrent connections of some Long Short-Term Memorys (LSTMs) and Gated Recurrent Units (GRUs). These do not appear to be present within any of our fitted models, where all initial states seem to invariably collapse to $\mathbf{0}$. Empirically, this holds both for models using our modified update equation eq. (4.3b) as well as models using the classic GRU update equation.

However, one can illustrate the dynamical behaviour mentioned in section 4.2 , as shown in fig. A.1, which plots the evolution of $\mathbf{h}^{t}$ given null inputs (i. e. $\mathbf{f}_{x}=\mathbf{0}$ ) for classic GRUs, the modified update rules, and Laurent and von Brecht [51]'s Chaos-Free Recurrent (CFR) cell. The first and second columns of figs. A.1a and A.1b plot $\mathbf{h}^{t}$ given a uniformly random $h_{0}$ drawn from $[-1,1]$. The first column illustrates the standard behaviour of the cell, while in the second column the input bias, $\mathbf{b}_{z}$ in eq. (4.3b), is set to $\mathbf{0}$ to remove the saturation effect. The third column illustrates the behaviour of the cell when $h^{0}=\mathbf{0}$, leaving only the interaction of $\mathbf{b}_{z}$ with the cell's recurrent connections. For the modified cell variant, these consists of the recurrent gate terms in eqs. (4.3c) and (4.3e).

The modified cells exhibit far more consistent behaviour, i. e. monotonic progression given null inputs, over time (fig. A.1d) than classic GRUs, while still being exhibiting some dynamical behaviour (figs. A.1b and A.1d). Note that unlike CFR cells, the modified GRU cell is not guaranteed to be consistent due to interactions between $\mathbf{b}_{z}$ and the gates' recurrence terms. This can be demonstrated by the following single unit cell whose steady state roughly alternates between $h=1$ and $h=2$ : 

$\mathbf{W}_{z}=\left[\begin{array}{ll}0 & 0\end{array}\right]$
$\mathbf{b}_{z}=[1]$
$\mathbf{W}_{u}=\left[\begin{array}{ll}0 & 0\end{array}\right]$
$\mathbf{b}_{u}=[100]$
$\mathbf{W}_{r}=\left[\begin{array}{ll}0 & -75\end{array}\right]$
$\mathbf{b}_{r}=[100]$

(A.1a) 


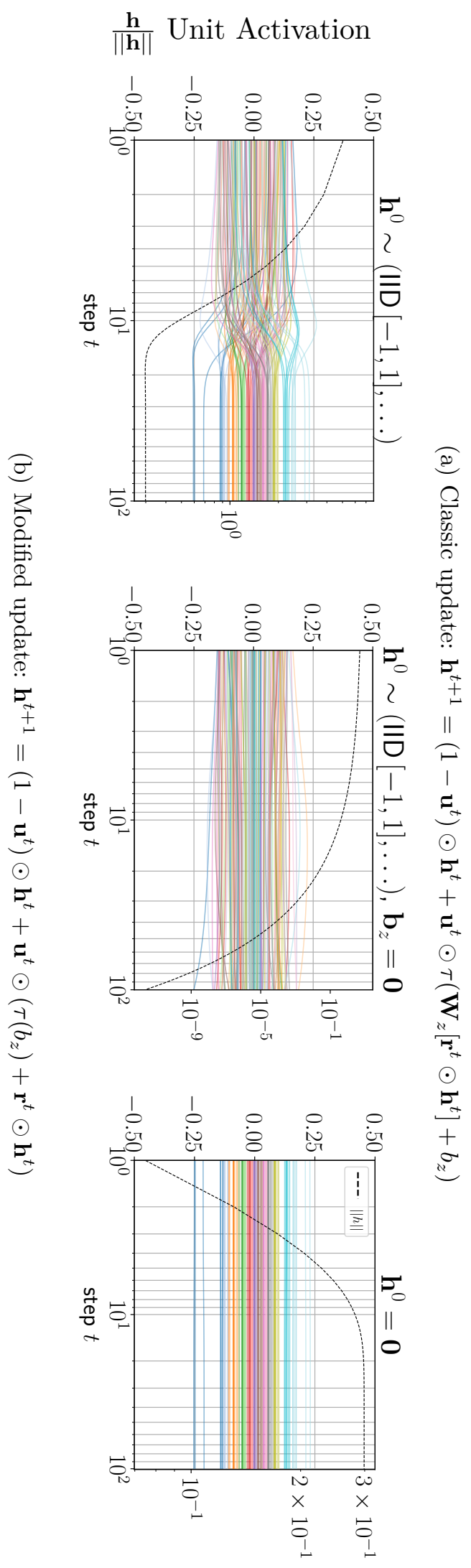

$\|\mathbf{h}\|$ $\frac{\mathbf{h}}{\|\mathbf{h}\|}$ Unit Activation
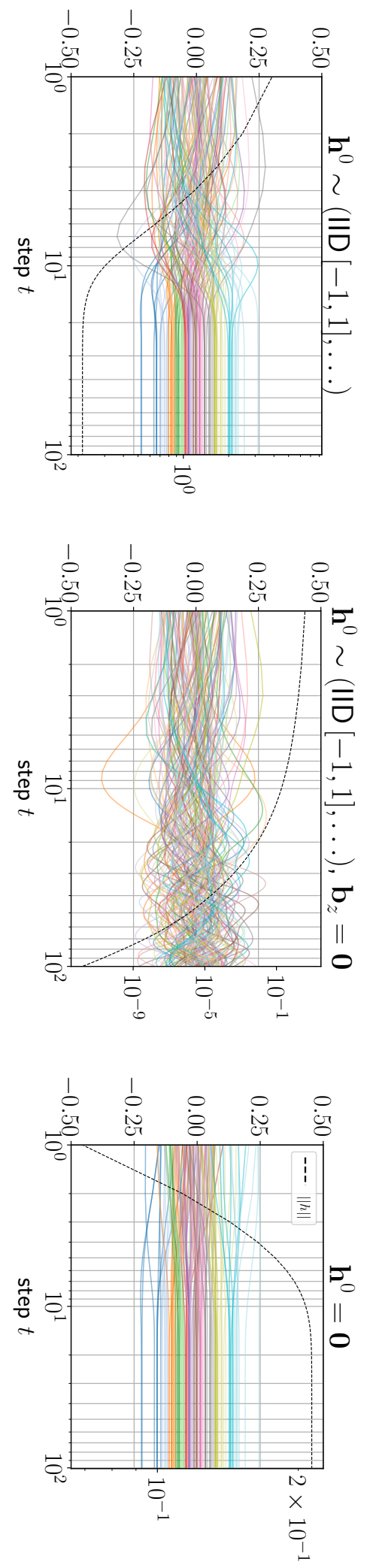

$\|\mathbf{h}\|$ 


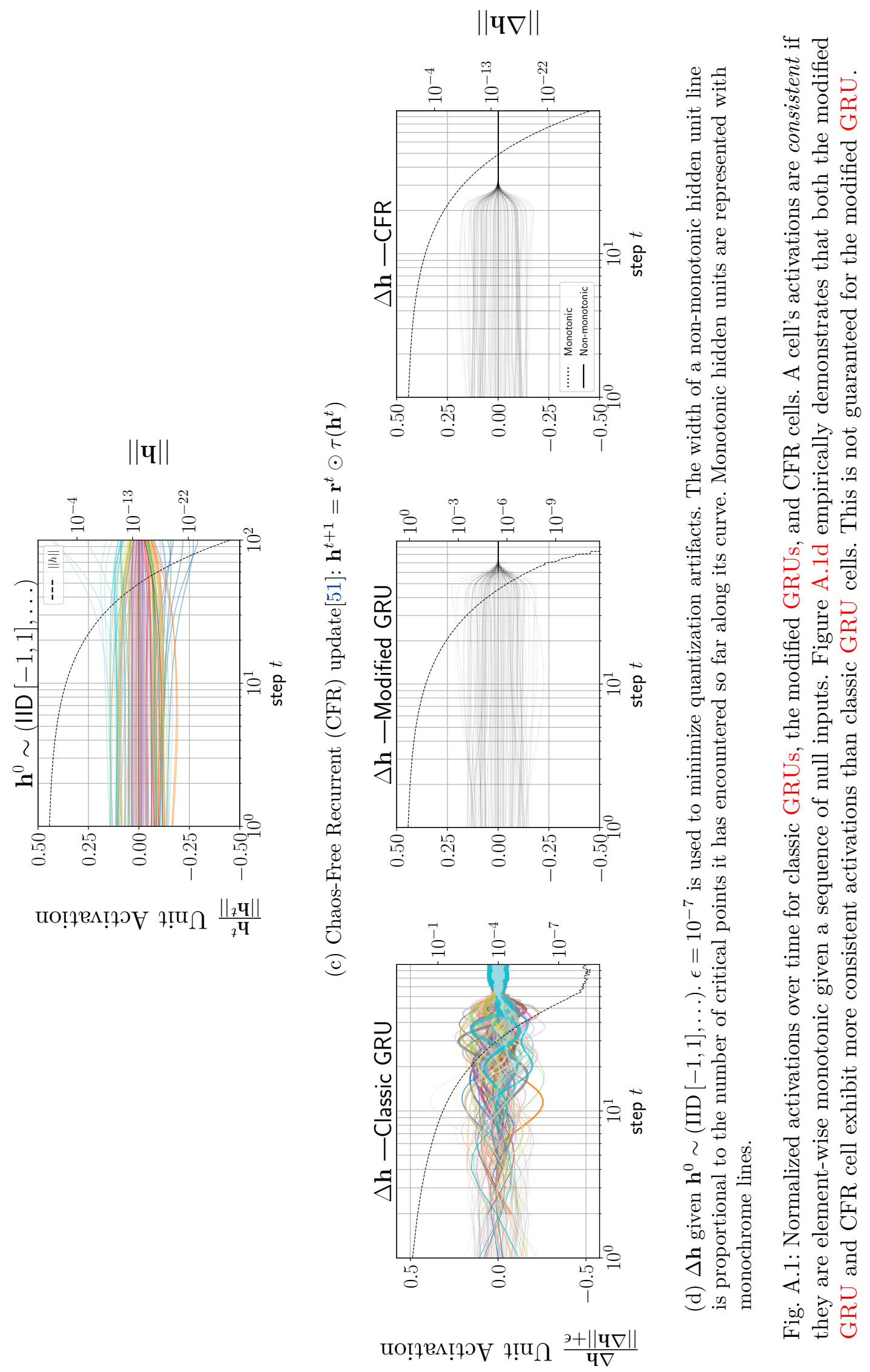




\section{APPENDIX B}

\begin{tabular}{|c|c|c|c|c|}
\hline \multirow[b]{2}{*}{ Project } & \multicolumn{2}{|c|}{ Samples } & \multicolumn{2}{|c|}{ Vertices } \\
\hline & Count & $\%$ Tot. & Count & $\%$ Tot. \\
\hline Apache Commons Codec & 677 & 0.43 & 22773 & 0.79 \\
\hline Apache Commons IO & 1378 & 0.88 & 23022 & 0.79 \\
\hline Apache Commons Logging & 283 & 0.18 & 4879 & 0.16 \\
\hline Apache HTTP & 4213 & 2.70 & 62579 & 2.17 \\
\hline Apache XML & 4414 & 2.83 & 69501 & 2.41 \\
\hline BouncyCastle & 24114 & 15.47 & 760247 & 26.38 \\
\hline Capnproto Runtime & 535 & 0.34 & 7244 & 0.25 \\
\hline dbus-java & 467 & 0.29 & 15542 & 0.53 \\
\hline DexLib & 4416 & 2.83 & 67883 & 2.35 \\
\hline ExtendJ & 16613 & 10.66 & 238920 & 8.29 \\
\hline FasterXML & 8382 & 5.37 & 139659 & 4.84 \\
\hline FuseSource hawtjni & 78 & 0.05 & 1644 & 0.05 \\
\hline FuseSource Jansi & 274 & 0.17 & 4333 & 0.15 \\
\hline Guava & 11944 & 7.66 & 121553 & 4.21 \\
\hline Jasmin & 135 & 0.08 & 14441 & 0.50 \\
\hline Java Unix Sockets & 105 & 0.06 & 1234 & 0.04 \\
\hline JBoss & 10519 & 6.75 & 167200 & 5.80 \\
\hline JCommander & 365 & 0.23 & 5829 & 0.20 \\
\hline Keycloak & 28430 & 18.24 & 403690 & 14.01 \\
\hline libpam4j & 76 & 0.04 & 766 & 0.02 \\
\hline Misc. Example Code & 104 & 0.06 & 1923 & 0.06 \\
\hline ObjectWeb ASM & 1796 & 1.15 & 46740 & 1.62 \\
\hline Polyglot & 3551 & 2.27 & 98378 & 3.41 \\
\hline Proprietary Tool 1 & 4428 & 2.84 & 43456 & 1.50 \\
\hline Scopt & 461 & 0.29 & 5377 & 0.18 \\
\hline SLF4J & 436 & 0.27 & 5023 & 0.17 \\
\hline Soot & 25752 & 16.52 & 504885 & 17.52 \\
\hline Twitter4J & 1778 & 1.14 & 35355 & 1.22 \\
\hline XMLPull & 85 & 0.05 & 6813 & 0.23 \\
\hline Total & 155809 & 100.0 & 2880889 & 100.0 \\
\hline
\end{tabular}

Table B.1: Population statistics for natural-negative code dataset. 


\section{APPENDix C \\ Metric Definitions}

Aside from cross-entropy, there are three metrics particularly suited for our evaluations: The receiver operating characteristics (ROC), average precision (AP), and the $\phi$ coefficient.

The most important of these for our task is the ROC, which is defined as:

$$
\mathrm{ROC}=\frac{T P R}{F P R}=\frac{\mathrm{TP}}{\mathrm{FP}} \frac{\mathrm{TN}+\mathrm{FP}}{\mathrm{TP}+\mathrm{FN}}
$$

A model with good $\mathrm{ROC}$ is robust in the sense that it is capable of ranking instances regardless of the classification threshold, which, in turn, can be adjusted for an unseen dataset.

The AP is defined as:

$$
\mathrm{AP}=\int_{0}^{1} P_{R}(r) \mathrm{d} r
$$

Where $P_{R}(r)$ is the precision at recall $r$. Equation (C.2) offers a more precise summary of the average precision than taking the area under the precision-recall curve. For situations where the recall is unavailable, i.e. partially labelled datasets (section 5.1.3 and section 5.4), the average precision weighed by threshold is used instead:

$$
\operatorname{APT}(i)=\int_{i} P_{T}(t) \mathrm{d} t
$$

Where $P_{T}(t)$ is the precision at for the classifier score threshold $t$ and $0 \leq i$ is a lower bound on the threshold selecting the subset of labelled samples considered.

The $\phi$ Coefficient is a correlation coefficient for two binary variables. Its domain is $[-1,1]$, where $1,-1$, and 0 indicate complete agreement, complete disagreement, and no correlation, respectively. It is also insensitive to the class sizes, providing an effective summary of a binary confusion matrix [64]. The $\phi$ Coefficient can be computed from a confusion matrix 
using:

$$
\phi \text { Coefficient }=\frac{\mathrm{TP} \cdot \mathrm{TN}-\mathrm{FP} \cdot \mathrm{FN}}{\sqrt{(\mathrm{TP}+\mathrm{FP})(\mathrm{TN}+\mathrm{FN})(\mathrm{TP}+\mathrm{FN})(\mathrm{TN}+\mathrm{FP})}}
$$




\section{APPENDIX D}

Table D.1: Feature-set ablation evaluation for primary dataset, using baseline and $k=5$ models. This table is equivalent to table 5.9 , but includes samples from natural-negative projects (section 5.1.2) in the reported metrics.

\begin{tabular}{|c|c|c|c|c|c|c|c|c|c|c|c|c|c|}
\hline \multirow{24}{*}{$\begin{array}{r}\text { Model } \\
\text { cbow }\end{array}$} & \multirow{3}{*}{$\begin{array}{l}\text { Scope } \\
\text { Sample }\end{array}$} & \multicolumn{4}{|c|}{ Features } & \multicolumn{2}{|c|}{ Cross-Entropy } & \multicolumn{2}{|c|}{ Avg. Precision } & \multicolumn{2}{|c|}{ Best $\phi$ Coeff. } & \multicolumn{2}{|c|}{ ROC AUC } \\
\hline & & Instr. & Type & Member & Const. & Train & Test & Train & Test & Train & $\overline{\text { Test }}$ & Train & Test \\
\hline & & & & & & $207 \cdot 10^{-3}$ & $208 \cdot 10^{-3}$ & 0.234 & 0.222 & 0.341 & 0.337 & 0.861 & 0.870 \\
\hline & & & & & $\checkmark$ & $35 \cdot 10^{-3}$ & $39 \cdot 10^{-3}$ & 0.954 & 0.937 & 0.859 & 0.848 & 0.997 & 0.997 \\
\hline & & & & $\checkmark$ & & $19 \cdot 10^{-3}$ & $16 \cdot 10^{-3}$ & 0.929 & 0.943 & 0.876 & 0.902 & 0.997 & 0.998 \\
\hline & & & $\checkmark$ & & & $64 \cdot 10^{-3}$ & $55 \cdot 10^{-3}$ & 0.723 & 0.749 & 0.714 & 0.743 & 0.987 & 0.989 \\
\hline & & $\checkmark$ & & & & $58 \cdot 10^{-3}$ & $56 \cdot 10^{-3}$ & 0.818 & 0.788 & 0.818 & 0.810 & 0.992 & 0.992 \\
\hline & & & & $\checkmark$ & $\checkmark$ & $7 \cdot 10^{-3}$ & $6 \cdot 10^{-3}$ & 0.998 & 0.998 & 0.973 & 0.977 & 1.000 & 1.000 \\
\hline & & & $\checkmark$ & $\checkmark$ & & $11 \cdot 10^{-3}$ & $10 \cdot 10^{-3}$ & 0.956 & 0.962 & 0.905 & 0.927 & 0.998 & 0.998 \\
\hline & & $\checkmark$ & & $\checkmark$ & & $13 \cdot 10^{-3}$ & $13 \cdot 10^{-3}$ & 0.960 & 0.969 & 0.899 & 0.911 & 0.998 & 0.998 \\
\hline & & & $\checkmark$ & $\checkmark$ & $\checkmark$ & $4 \cdot 10^{-3}$ & $3 \cdot 10^{-3}$ & 0.999 & 0.993 & 0.985 & 0.989 & 1.000 & 1.000 \\
\hline & & $\checkmark$ & $\checkmark$ & $\checkmark$ & & $9 \cdot 10^{-3}$ & $6 \cdot 10^{-3}$ & 0.983 & 0.983 & 0.939 & 0.957 & 0.999 & 0.999 \\
\hline & & $\checkmark$ & $\checkmark$ & $\checkmark$ & $\checkmark$ & $3 \cdot 10^{-3}$ & $1 \cdot 10^{-3}$ & 0.999 & 1.000 & 0.987 & 0.991 & 1.000 & 1.000 \\
\hline & Vertex & & & & & $35 \cdot 10^{-3}$ & $36 \cdot 10^{-3}$ & 0.016 & 0.015 & 0.062 & 0.059 & 0.729 & 0.719 \\
\hline & & & & & $\checkmark$ & $16 \cdot 10^{-3}$ & $17 \cdot 10^{-3}$ & 0.408 & 0.411 & 0.426 & 0.422 & 0.976 & 0.977 \\
\hline & & & & $\checkmark$ & & $12 \cdot 10^{-3}$ & $11 \cdot 10^{-3}$ & 0.627 & 0.657 & 0.604 & 0.621 & 0.988 & 0.989 \\
\hline & & & $\checkmark$ & & & $16 \cdot 10^{-3}$ & $16 \cdot 10^{-3}$ & 0.359 & 0.408 & 0.396 & 0.432 & 0.980 & 0.983 \\
\hline & & $\checkmark$ & & & & $15 \cdot 10^{-3}$ & $15 \cdot 10^{-3}$ & 0.335 & 0.310 & 0.376 & 0.387 & 0.984 & 0.984 \\
\hline & & & & $\checkmark$ & $\checkmark$ & $6 \cdot 10^{-3}$ & $5 \cdot 10^{-3}$ & 0.875 & 0.894 & 0.809 & 0.825 & 0.997 & 0.997 \\
\hline & & & $\checkmark$ & $\checkmark$ & & $7 \cdot 10^{-3}$ & $7 \cdot 10^{-3}$ & 0.760 & 0.788 & 0.674 & 0.693 & 0.996 & 0.997 \\
\hline & & $\checkmark$ & & $\checkmark$ & & $7 \cdot 10^{-3}$ & $7 \cdot 10^{-3}$ & 0.757 & 0.784 & 0.659 & 0.679 & 0.996 & 0.997 \\
\hline & & & $\checkmark$ & $\checkmark$ & $\checkmark$ & $3 \cdot 10^{-3}$ & $2 \cdot 10^{-3}$ & 0.946 & 0.955 & 0.849 & 0.859 & 0.999 & 1.000 \\
\hline & & $\checkmark$ & $\checkmark$ & $\checkmark$ & & $6 \cdot 10^{-3}$ & $5 \cdot 10^{-3}$ & 0.821 & 0.852 & 0.715 & 0.742 & 0.998 & 0.998 \\
\hline & & $\checkmark$ & $\checkmark$ & $\checkmark$ & $\checkmark$ & $2 \cdot 10^{-3}$ & $2 \cdot 10^{-3}$ & 0.960 & 0.966 & 0.865 & 0.876 & 1.000 & 1.000 \\
\hline $\mathrm{k}=0$ & Sample & & & & & $222 \cdot 10^{-3}$ & $222 \cdot 10^{-3}$ & 0.045 & 0.046 & 0.007 & 0.000 & 0.488 & 0.500 \\
\hline & & & & & $\checkmark$ & $52 \cdot 10^{-3}$ & $51 \cdot 10^{-3}$ & 0.668 & 0.670 & 0.642 & 0.653 & 0.974 & 0.976 \\
\hline & & & & $\checkmark$ & & $37 \cdot 10^{-3}$ & $34 \cdot 10^{-3}$ & 0.661 & 0.662 & 0.639 & 0.648 & 0.975 & 0.980 \\
\hline & & & $\checkmark$ & & & $93 \cdot 10^{-3}$ & $94 \cdot 10^{-3}$ & 0.394 & 0.383 & 0.413 & 0.450 & 0.926 & 0.934 \\
\hline & & $\checkmark$ & & & & $162 \cdot 10^{-3}$ & $159 \cdot 10^{-3}$ & 0.111 & 0.119 & 0.261 & 0.277 & 0.812 & 0.821 \\
\hline & & & & $\checkmark$ & $\checkmark$ & $25 \cdot 10^{-3}$ & $24 \cdot 10^{-3}$ & 0.715 & 0.701 & 0.665 & 0.690 & 0.985 & 0.986 \\
\hline & & & $\checkmark$ & $\checkmark$ & & $37 \cdot 10^{-3}$ & $33 \cdot 10^{-3}$ & 0.650 & 0.650 & 36 & 0.648 & 0.976 & 0.979 \\
\hline & & $\checkmark$ & & $\checkmark$ & & $37 \cdot 10^{-3}$ & $36 \cdot 10^{-3}$ & 0.648 & 0.666 & 0.639 & 0.653 & 0.978 & 0.981 \\
\hline & & & $\checkmark$ & $\checkmark$ & $\checkmark$ & $24 \cdot 10^{-3}$ & $24 \cdot 10^{-3}$ & 0.700 & 0.681 & 0.677 & 0.693 & 0.985 & 0.986 \\
\hline & & $\checkmark$ & $\checkmark$ & $\checkmark$ & & $36 \cdot 10^{-3}$ & $36 \cdot 10^{-3}$ & 0.651 & 0.661 & 0.641 & 0.651 & 0.978 & 0.981 \\
\hline & & $\checkmark$ & $\checkmark$ & $\checkmark$ & $\checkmark$ & $25 \cdot 10^{-3}$ & $24 \cdot 10^{-3}$ & 0.711 & 0.695 & 0.674 & 0.676 & 0.985 & 0.986 \\
\hline & Vertex & & & & & $38 \cdot 10^{-3}$ & $38 \cdot 10^{-3}$ & 0.007 & 0.008 & 0.000 & 0.000 & 0.472 & 0.500 \\
\hline & & & & & $\checkmark$ & $32 \cdot 10^{-3}$ & $33 \cdot 10^{-3}$ & 0.151 & 0.146 & 0.299 & 0.302 & 0.659 & 0.672 \\
\hline & & & & $\checkmark$ & & $26 \cdot 10^{-3}$ & $25 \cdot 10^{-3}$ & 0.209 & 0.203 & 0.281 & 0.299 & 0.799 & 0.815 \\
\hline & & & $\checkmark$ & & & $27 \cdot 10^{-3}$ & $28 \cdot 10^{-3}$ & 0.085 & 0.083 & 0.186 & 0.199 & 0.893 & 0.907 \\
\hline & & $\checkmark$ & & & & $30 \cdot 10^{-3}$ & $32 \cdot 10^{-3}$ & 0.026 & 0.027 & 0.117 & 0.121 & 0.842 & 0.843 \\
\hline & & & & $\checkmark$ & $\checkmark$ & $18 \cdot 10^{-3}$ & $19 \cdot 10^{-3}$ & 0.357 & 0.350 & 0.408 & 0.423 & 0.913 & 0.924 \\
\hline & & & $d$ & $\checkmark$ & & $21 \cdot 10^{-3}$ & $20 \cdot 10^{-3}$ & & 0.2 & 86 & 0.304 & 0.943 & 0.956 \\
\hline & & $d$ & & 2 & & $22 \cdot 10^{-3}$ & $22 \cdot 10^{-3}$ & & 0.223 & 0.285 & & 0.943 & 0.947 \\
\hline & & & $\checkmark$ & $\checkmark$ & $\checkmark$ & $16 \cdot 10^{-3}$ & $15 \cdot 10^{-3}$ & 0.376 & 0.366 & 0.411 & 0.422 & 0.973 & 0.977 \\
\hline & & $\checkmark$ & $\checkmark$ & $\checkmark$ & & $20 \cdot 10^{-3}$ & $21 \cdot 10^{-3}$ & 0.240 & 0.231 & 0.297 & 0.307 & 0.958 & 0.960 \\
\hline & & $\checkmark$ & $\checkmark$ & $\checkmark$ & $\checkmark$ & $16 \cdot 10^{-3}$ & $14 \cdot 10^{-3}$ & 0.378 & 0.379 & 0.413 & 0.438 & 0.976 & 0.980 \\
\hline $\mathrm{k}=5$ & Sample & & & & & $10 \cdot 10^{-3}$ & $14 \cdot 10^{-3}$ & 0.925 & 0.874 & 0.833 & 0.826 & 0.994 & 0.989 \\
\hline & & & & & $\checkmark$ & $3 \cdot 10^{-3}$ & $3 \cdot 10^{-3}$ & 0.996 & 0.989 & 0.964 & 0.951 & 1.000 & 0.999 \\
\hline & & & & $\checkmark$ & & $4 \cdot 10^{-3}$ & $5 \cdot 10^{-3}$ & 0.975 & 0.961 & 0.910 & 0.922 & 0.998 & 0.997 \\
\hline & & & $\checkmark$ & & & $8 \cdot 10^{-3}$ & $10 \cdot 10^{-3}$ & 0.935 & 0.939 & 0.861 & 0.862 & 0.997 & 0.996 \\
\hline & & $\checkmark$ & & & & $5 \cdot 10^{-3}$ & $6 \cdot 10^{-3}$ & 0.972 & 0.961 & 0.888 & 0.882 & 0.998 & 0.998 \\
\hline & & & & $\checkmark$ & $\checkmark$ & $3 \cdot 10^{-3}$ & $3 \cdot 10^{-3}$ & 0.995 & 0.994 & 0.966 & 0.989 & 1.000 & 0.999 \\
\hline & & & 2 & 2 & & $4 \cdot 10^{-3}$ & $4 \cdot 10^{-3}$ & 0.979 & 0.971 & 0.919 & 0.919 & 0.998 & 0.998 \\
\hline & & $\checkmark$ & & $\checkmark$ & & $2 \cdot 10^{-3}$ & $2 \cdot 10^{-3}$ & 0.994 & 0.987 & 0.954 & 0.948 & 1.000 & 0.999 \\
\hline & & & $\checkmark$ & $\checkmark$ & $\checkmark$ & $1 \cdot 10^{-3}$ & $3 \cdot 10^{-3}$ & 0.998 & 0.994 & 0.975 & 0.981 & 1.000 & 0.998 \\
\hline & & $\checkmark$ & $\checkmark$ & $\checkmark$ & & $1 \cdot 10^{-3}$ & $2 \cdot 10^{-3}$ & 0.997 & 0.994 & 0.968 & 0.968 & 1.000 & 1.000 \\
\hline & & $\checkmark$ & $\checkmark$ & $\checkmark$ & $\checkmark$ & $2 \cdot 10^{-3}$ & $4 \cdot 10^{-3}$ & 0.995 & 0.993 & 0.953 & 0.969 & 1.000 & 0.999 \\
\hline & Vertex & & & & & $4 \cdot 10^{-3}$ & $5 \cdot 10^{-3}$ & 0.882 & 0.840 & 0.807 & 0.802 & 0.999 & 0.997 \\
\hline & & & & & $\checkmark$ & $1 \cdot 10^{-3}$ & $2 \cdot 10^{-3}$ & 0.986 & 0.979 & 0.926 & 0.916 & 1.000 & 1.000 \\
\hline & & & & $\checkmark$ & & $2 \cdot 10^{-3}$ & $2 \cdot 10^{-3}$ & 0.959 & 0.953 & 0.915 & 0.926 & 1.000 & 0.999 \\
\hline & & & $\checkmark$ & & & $4 \cdot 10^{-3}$ & $4 \cdot 10^{-3}$ & 0.889 & 0.896 & 0.827 & 0.825 & 0.999 & 0.998 \\
\hline & & $\checkmark$ & & & & $2 \cdot 10^{-3}$ & $3 \cdot 10^{-3}$ & 0.951 & 0.938 & 0.867 & 0.851 & 1.000 & 0.999 \\
\hline & & & & $\checkmark$ & $\checkmark$ & $624 \cdot 10^{-6}$ & $456 \cdot 10^{-6}$ & 0.996 & 0.996 & 0.977 & 0.985 & 1.000 & 1.000 \\
\hline & & & $\checkmark$ & $\checkmark$ & & $2 \cdot 10^{-3}$ & $1 \cdot 10^{-3}$ & 0.968 & 0.958 & 0.920 & 0.930 & 1.000 & 0.999 \\
\hline & & $d$ & & 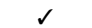 & & $894 \cdot 10^{-6}$ & $683 \cdot 10^{-6}$ & 0.994 & 0.990 & 0.957 & 0.964 & 1.000 & 1.000 \\
\hline & & & $\checkmark$ & $\checkmark$ & $\checkmark$ & $402 \cdot 10^{-6}$ & $619 \cdot 10^{-6}$ & 0.998 & 0.996 & 0.981 & 0.984 & 1.000 & 0.999 \\
\hline & & $\checkmark$ & $\checkmark$ & $\checkmark$ & & $793 \cdot 10^{-6}$ & $534 \cdot 10^{-6}$ & 0.995 & 0.996 & 0.969 & 0.978 & 1.000 & 1.000 \\
\hline & & $\checkmark$ & $\checkmark$ & $\checkmark$ & $\checkmark$ & $597 \cdot 10^{-6}$ & $555 \cdot 10^{-6}$ & 0.996 & 0.997 & 0.969 & 0.973 & 1.000 & 1.000 \\
\hline
\end{tabular}




\section{Bibliography}

[1] Jvm specification, 4.10.2. verification by type inference. https://docs.oracle.com/ javase/specs/jvms/se7/html/jvms-4.html\#jvms-4.10.2, . Accessed: 2018-10-06. 7

[2] Jvm specification, 4.10.1.2. verification type system. https://docs.oracle.com/ javase/specs/jvms/se7/html/jvms-4.html\#jvms-4.10.1.2, . Accessed: 2018-10-06. 7

[3] Miltiadis Allamanis and Marc Brockschmidt. Smartpaste: Learning to adapt source code. arXiv preprint arXiv:1705.07867, 2017. 18

[4] Miltiadis Allamanis, Earl T Barr, Christian Bird, and Charles Sutton. Suggesting accurate method and class names. In Proceedings of the 2015 10th Joint Meeting on Foundations of Software Engineering, pages 38-49. ACM, 2015. 17

[5] Miltiadis Allamanis, Earl T Barr, Premkumar Devanbu, and Charles Sutton. A survey of machine learning for big code and naturalness. ACM Computing Surveys (CSUR), 51(4):81, 2018. 16, 17

[6] Miltiadis Allamanis, Marc Brockschmidt, and Mahmoud Khademi. Learning to represent programs with graphs. In International Conference on Learning Representations, 2018. URL https://openreview.net/forum?id=BJOFETxR-. 13, 16, 18, 29, 30

[7] Nada Amin and Ross Tate. Java and scala's type systems are unsound: the existential crisis of null pointers. In Acm Sigplan Notices, volume 51, pages 838-848. ACM, 2016. 7

[8] Jaume Amores. Multiple instance classification: Review, taxonomy and comparative study. Artificial Intelligence, 201:81-105, 2013. 14

[9] Martin Arjovsky, Amar Shah, and Yoshua Bengio. Unitary evolution recurrent neural networks. In International Conference on Machine Learning, pages 1120-1128, 2016. 11

[10] Phillipe Arteau. Find security bugs. URL https://find-sec-bugs.github.io/. 46, 51

[11] David Balduzzi and Muhammad Ghifary. Strongly-typed recurrent neural networks. In International Conference on Machine Learning, pages 1292-1300, 2016. 8

[12] Anindya Banerjee, Benjamin Livshits, Aditya Nori, Sriram Rajamani, and Ben Livshits. Merlin: Specification inference for explicit information flow problems. Technical report, December 2008. URL https://www.microsoft.com/en-us/research/publication/ merlin-specification-inference-for-explicit-information-flow-problems/. 21,57

[13] Rohan Bavishi, Michael Pradel, and Koushik Sen. Context2name: A deep learning-based approach to infer natural variable names from usage contexts. 2017. 18 
[14] James Bradbury, Stephen Merity, Caiming Xiong, and Richard Socher. Quasi-recurrent neural networks. arXiv preprint arXiv:1611.01576, 2016. 31

[15] Preston Briggs, Keith D Cooper, Timothy J Harvey, and L Taylor Simpson. Practical improvements to the construction and destruction of static single assignment form. Software: Practice and Experience, 28(8):859-881, 1998. 6

[16] Marc Brockschmidt, Yuxin Chen, Byron Cook, Pushmeet Kohli, and Daniel Tarlow. Learning to decipher the heap for program verification. In Workshop on Constructive Machine Learning at the International Conference on Machine Learning (CMLICML), 2015. 21

[17] Asuncion Castano and Francisco Casacuberta. A connectionist approach to machine translation. In Fifth European Conference on Speech Communication and Technology, 1997. 11

[18] Chih-Chung Chang and Chih-Jen Lin. Libsvm: a library for support vector machines. ACM transactions on intelligent systems and technology (TIST), 2(3):27, 2011. 75

[19] Kyunghyun Cho, Bart Van Merriënboer, Caglar Gulcehre, Dzmitry Bahdanau, Fethi Bougares, Holger Schwenk, and Yoshua Bengio. Learning phrase representations using rnn encoder-decoder for statistical machine translation. arXiv preprint arXiv:1406.1078, 2014. 11, 74

[20] Luigi P Cordella, Pasquale Foggia, Carlo Sansone, and Mario Vento. A (sub) graph isomorphism algorithm for matching large graphs. IEEE transactions on pattern analysis and machine intelligence, 26(10):1367-1372, 2004. 44

[21] Ron Cytron, Jeanne Ferrante, Barry K Rosen, Mark N Wegman, and F Kenneth Zadeck. Efficiently computing static single assignment form and the control dependence graph. ACM Transactions on Programming Languages and Systems (TOPLAS), 13(4):451-490, 1991. 6

[22] Andrew M Dai and Quoc V Le. Semi-supervised sequence learning. In Advances in neural information processing systems, pages 3079-3087, 2015. 18

[23] Michaël Defferrard, Xavier Bresson, and Pierre Vandergheynst. Convolutional neural networks on graphs with fast localized spectral filtering. In D. D. Lee, M. Sugiyama, U. V. Luxburg, I. Guyon, and R. Garnett, editors, Advances in Neural Information Processing Systems 29, pages 3844-3852. Curran Associates, Inc., 2016. 11, 13

[24] Florian Deissenboeck and Markus Pizka. Concise and consistent naming. Software Quality Journal, 14(3):261-282, 2006. 17

[25] Wolfgang Ertel, Johann M Ph Schumann, and Christian B Suttner. Learning heuristics for a theorem prover using back propagation. In 5. Österreichische Artificial-IntelligenceTagung, pages 87-95. Springer, 1989. 19

[26] Paolo Frasconi, Marco Gori, and Alessandro Sperduti. A general framework for adaptive processing of data structures. IEEE transactions on Neural Networks, 9(5):768-786, 1998. 11, 13, 20 
[27] Ken-ichi Funahashi and Yuichi Nakamura. Approximation of dynamical systems by continuous time recurrent neural networks. Neural networks, 6(6):801-806, 1993. 8, 11

[28] Mark Gabel and Zhendong Su. A study of the uniqueness of source code. In Proceedings of the eighteenth ACM SIGSOFT international symposium on Foundations of software engineering, pages 147-156. ACM, 2010. 17, 20

[29] Xavier Glorot and Yoshua Bengio. Understanding the difficulty of training deep feedforward neural networks. In Proceedings of the thirteenth international conference on artificial intelligence and statistics, pages 249-256, 2010. 45

[30] Christoph Goller. A connectionist control component for the theorem prover setheo. In ECAI94 Workshop on Combining Symbolic and Connectionist Processing, 1994. 19

[31] Christoph Goller and Andreas Kuchler. Learning task-dependent distributed representations by backpropagation through structure. In Neural Networks, 1996., IEEE International Conference on, volume 1, pages 347-352. IEEE, 1996. 16, 20

[32] Ian J Goodfellow, Mehdi Mirza, Da Xiao, Aaron Courville, and Yoshua Bengio. An empirical investigation of catastrophic forgetting in gradient-based neural networks. arXiv preprint arXiv:1312.6211, 2013. 9

[33] Klaus Greff, Rupesh K Srivastava, Jan Koutník, Bas R Steunebrink, and Jürgen Schmidhuber. Lstm: A search space odyssey. IEEE transactions on neural networks and learning systems, 28(10):2222-2232, 2017. 11

[34] Aditya Grover and Jure Leskovec. node2vec: Scalable feature learning for networks. In Proceedings of the 22nd ACM SIGKDD international conference on Knowledge discovery and data mining, pages 855-864. ACM, 2016. 12

[35] Will Hamilton, Zhitao Ying, and Jure Leskovec. Inductive representation learning on large graphs. In Advances in Neural Information Processing Systems, pages 1024-1034, 2017. 13

[36] Mikael Henaff, Joan Bruna, and Yann LeCun. Deep convolutional networks on graphstructured data. arXiv preprint arXiv:1506.05163, 2015. 11

[37] Jordan Henkel, Shuvendu K. Lahiri, Ben Liblit, and Thomas Reps. Code vectors: Understanding programs through embedded abstracted symbolic traces. In Corina Pasareanu and Alessandro Garcia, editors, Proceedings of the 26th ACM SIGSOFT International Symposium on Foundations of Software Engineering, Lake Buena Vista, Florida, November 2018. ACM SIGSOFT. 17, 19, 20

[38] Aurélie Herbelot and Marco Baroni. High-risk learning: acquiring new word vectors from tiny data. In Proceedings of the 2017 Conference on Empirical Methods in Natural Language Processing, pages 304-309, 2017. 57

[39] Abram Hindle, Earl T Barr, Zhendong Su, Mark Gabel, and Premkumar Devanbu. On the naturalness of software. In Software Engineering (ICSE), 2012 34th International Conference on, pages 837-847. IEEE, 2012. 17, 20 
[40] Sepp Hochreiter and Jurgen Schmidhuber. Bridging long time lags by weight guessing and "long short-term memory". Spatiotemporal models in biological and artificial systems, 37:65-72, 1996. 11

[41] Sepp Hochreiter and Jürgen Schmidhuber. Long short-term memory. Neural computation, 9(8):1735-1780, 1997. 11, 75

[42] Kurt Hornik. Some new results on neural network approximation. Neural networks, 6 (8):1069-1072, 1993. 8

[43] Kurt Hornik, Maxwell Stinchcombe, and Halbert White. Multilayer feedforward networks are universal approximators. Neural networks, 2(5):359-366, 1989. 8, 11

[44] Mohit Iyyer, Varun Manjunatha, Jordan Boyd-Graber, and Hal Daumé III. Deep unordered composition rivals syntactic methods for text classification. In Proceedings of the 53rd Annual Meeting of the Association for Computational Linguistics and the 7th International Joint Conference on Natural Language Processing (Volume 1: Long Papers), volume 1, pages 1681-1691, 2015. 30

[45] René Just, Darioush Jalali, and Michael D Ernst. Defects4j: A database of existing faults to enable controlled testing studies for java programs. In Proceedings of the 2014 International Symposium on Software Testing and Analysis, pages 437-440. ACM, 2014. 16

[46] Cezary Kaliszyk, François Chollet, and Christian Szegedy. Holstep: A machine learning dataset for higher-order logic theorem proving. International Conference on Learning Representations, 2017. 13, 16, 20

[47] Andrej Karpathy, Justin Johnson, and Li Fei-Fei. Visualizing and understanding recurrent networks. arXiv preprint arXiv:1506.02078, 2015. 21

[48] Yoon Kim. Convolutional neural networks for sentence classification. In Proceedings of the 2014 Conference on Empirical Methods in Natural Language Processing (EMNLP), pages $1746-1751,2014.31$

[49] Ugur Koc, Parsa Saadatpanah, Jeffrey S Foster, and Adam A Porter. Learning a classifier for false positive error reports emitted by static code analysis tools. In Proceedings of the 1st ACM SIGPLAN International Workshop on Machine Learning and Programming Languages, pages 35-42. ACM, 2017. 16

[50] Ted Kremenek, Andrew Y Ng, and Dawson R Engler. A factor graph model for software bug finding. In IJCAI, pages 2510-2516, 2007. 21

[51] Thomas Laurent and James von Brecht. A recurrent neural network without chaos. International Conference on Learning Representations, 2016. 33, 58, 61

[52] Quoc V Le, Navdeep Jaitly, and Geoffrey E Hinton. A simple way to initialize recurrent networks of rectified linear units. arXiv preprint arXiv:1504.00941, 2015. 45

[53] Nicolas Le Roux and Yoshua Bengio. Deep belief networks are compact universal approximators. Neural computation, 22(8):2192-2207, 2010. 8 
[54] Jian Li, Pinjia He, Jieming Zhu, and Michael R Lyu. Software defect prediction via convolutional neural network. In Software Quality, Reliability and Security (QRS), 2017 IEEE International Conference on, pages 318-328. IEEE, 2017. 19

[55] Yujia Li, Daniel Tarlow, Marc Brockschmidt, and Richard Zemel. Gated graph sequence neural networks. 2016. 11, 13, 18, 21

[56] Charith Mendis, Saman Amarasinghe, and Michael Carbin. Ithemal: Accurate, portable and fast basic block throughput estimation using deep neural networks. arXiv preprint arXiv:1808.07412, 2018. 20

[57] MITRE. Cwe - common weakness enumeration, 2018. URL https://cwe.mitre.org. 74

[58] Andriy Mnih and Geoffrey Hinton. Three new graphical models for statistical language modelling. In Proceedings of the 24th international conference on Machine learning, pages 641-648. ACM, 2007. 17

[59] Martin Monperrus and Mira Mezini. Detecting missing method calls as violations of the majority rule. ACM Transactions on Software Engineering and Methodology (TOSEM), 22(1):7, 2013. 20

[60] NIST. Software assurance reference dataset, 2018. URL https://samate.nist.gov/ SARD/testsuite.php. ii, 2, 16, 37, 56

[61] Jooyoung Park and Irwin W Sandberg. Universal approximation using radial-basisfunction networks. Neural computation, 3(2):246-257, 1991. 8

[62] Jeffrey Pennington, Richard Socher, and Christopher Manning. Glove: Global vectors for word representation. In Proceedings of the 2014 conference on empirical methods in natural language processing (EMNLP), pages 1532-1543, 2014. 19

[63] Bryan Perozzi, Rami Al-Rfou, and Steven Skiena. Deepwalk: Online learning of social representations. In Proceedings of the 20th ACM SIGKDD international conference on Knowledge discovery and data mining, pages 701-710. ACM, 2014. 12

[64] David Martin Powers. Evaluation: from precision, recall and f-measure to roc, informedness, markedness and correlation. 2011. 63

[65] Michael Pradel and Koushik Sen. Deep learning to find bugs. 2017. 16, 19

[66] Jiezhong Qiu, Yuxiao Dong, Hao Ma, Jian Li, Kuansan Wang, and Jie Tang. Network embedding as matrix factorization: Unifying deepwalk, line, pte, and node2vec. In Proceedings of the Eleventh ACM International Conference on Web Search and Data Mining, pages 459-467. ACM, 2018. 12

[67] Baishakhi Ray, Vincent Hellendoorn, Saheel Godhane, Zhaopeng Tu, Alberto Bacchelli, and Premkumar Devanbu. On the" naturalness" of buggy code. In Software Engineering (ICSE), 2016 IEEE/ACM 38th International Conference on, pages 428-439. IEEE, 2016. 20

[68] Veselin Raychev, Martin Vechev, and Andreas Krause. Predicting program properties from big code. In ACM SIGPLAN Notices, volume 50, pages 111-124. ACM, 2015. 18 
[69] John C Reynolds. Separation logic: A logic for shared mutable data structures. In Logic in Computer Science, 2002. Proceedings. 17th Annual IEEE Symposium on, pages 55-74. IEEE, 2002. 21

[70] Barry K Rosen, Mark N Wegman, and F Kenneth Zadeck. Global value numbers and redundant computations. In Proceedings of the 15th ACM SIGPLAN-SIGACT symposium on Principles of programming languages, pages 12-27. ACM, 1988. 6

[71] Frank Rosenblatt. The perceptron: a probabilistic model for information storage and organization in the brain. Psychological review, 65(6):386, 1958. 10

[72] Rebecca L Russell, Louis Kim, Lei H Hamilton, Tomo Lazovich, Jacob A Harer, Onur Ozdemir, Paul M Ellingwood, and Marc W McConley. Automated vulnerability detection in source code using deep representation learning. arXiv preprint arXiv:180\%.04320, 2018. 19

[73] Arthur L Samuel. Some studies in machine learning using the game of checkers. IBM Journal of research and development, 3(3):210-229, 1959. 19

[74] Franco Scarselli, Marco Gori, Ah Chung Tsoi, Markus Hagenbuchner, and Gabriele Monfardini. The graph neural network model. IEEE Transactions on Neural Networks, 20(1):61-80, 2009. 11, 13, 16, 20

[75] Jürgen Schmidhuber. Deep learning in neural networks: An overview. Neural networks, 61:85-117, 2015. 9, 10, 11

[76] Hava T Siegelmann and Eduardo D Sontag. On the computational power of neural nets. Journal of computer and system sciences, 50(1):132-150, 1995. 11

[77] Alessandro Sperduti and Antonina Starita. Supervised neural networks for the classification of structures. IEEE Transactions on Neural Networks, 8(3):714-735, 1997. 20

[78] Alessandro Sperduti, Antonina Starita, and Christoph Goller. Learning distributed representations for the classification of terms. In IJCAI, pages 509-517. Citeseer, 1995. 20

[79] Nitish Srivastava, Geoffrey Hinton, Alex Krizhevsky, Ilya Sutskever, and Ruslan Salakhutdinov. Dropout: a simple way to prevent neural networks from overfitting. The Journal of Machine Learning Research, 15(1):1929-1958, 2014. 9, 30

[80] Christian Suttner and Wolfgang Ertel. Automatic acquisition of search guiding heuristics. In International Conference on Automated Deduction, pages 470-484. Springer, 1990. 19

[81] Kai Sheng Tai, Richard Socher, and Christopher D. Manning. Improved semantic representations from tree-structured long short-term memory networks. In Proceedings of the 53rd Annual Meeting of the Association for Computational Linguistics and the 7th International Joint Conference on Natural Language Processing (Volume 1: Long Papers), pages 1556-1566, Beijing, China, July 2015. Association for Computational Linguistics. URL http://www.aclweb.org/anthology/P15-1150. 11 
[82] Duyu Tang, Bing Qin, and Ting Liu. Document modeling with gated recurrent neural network for sentiment classification. In Proceedings of the 2015 conference on empirical methods in natural language processing, pages 1422-1432, 2015. 11, 31

[83] Tijmen Tieleman and Geoffrey Hinton. Lecture 6.5 - rmsprop, 2012. 34, 45

[84] Raja Vallée-Rai, Phong Co, Etienne Gagnon, Laurie Hendren, Patrick Lam, and Vijay Sundaresan. Soot - a java bytecode optimization framework. In Proceedings of the 1999 Conference of the Centre for Advanced Studies on Collaborative Research, CASCON '99, pages 13-. IBM Press, 1999. URL http://dl.acm.org/citation.cfm?id=781995. 782008. 23

[85] Ashish Vaswani, Noam Shazeer, Niki Parmar, Jakob Uszkoreit, Llion Jones, Aidan N Gomez, Łukasz Kaiser, and Illia Polosukhin. Attention is all you need. In Advances in Neural Information Processing Systems, pages 5998-6008, 2017. 14

[86] S Vichy N Vishwanathan, Nicol N Schraudolph, Risi Kondor, and Karsten M Borgwardt. Graph kernels. Journal of Machine Learning Research, 11(Apr):1201-1242, 2010. 15, 75

[87] Eugene Vorontsov, Chiheb Trabelsi, Samuel Kadoury, and Chris Pal. On orthogonality and learning recurrent networks with long term dependencies. arXiv preprint arXiv:1702.00071, 2017. 11

[88] Song Wang, Taiyue Liu, and Lin Tan. Automatically learning semantic features for defect prediction. In Proceedings of the 38th International Conference on Software Engineering, pages 297-308. ACM, 2016. 19

[89] Andrzej Wasylkowski, Andreas Zeller, and Christian Lindig. Detecting object usage anomalies. In Proceedings of the the 6th joint meeting of the European software engineering conference and the ACM SIGSOFT symposium on The foundations of software engineering, pages 35-44. ACM, 2007. 20

[90] Open web application security project. Owasp - benchmark, 2016. URL https: //rawgit.com/OWASP/Benchmark/master/scorecard/OWASP_Benchmark_Home.html. 51,54

[91] Open web application security project. Owasp - benchmark, 2018. URL https: //www. owasp.org/index.php/Benchmark. 16

[92] Martin White, Michele Tufano, Christopher Vendome, and Denys Poshyvanyk. Deep learning code fragments for code clone detection. In Proceedings of the 31st IEEE/ACM International Conference on Automated Software Engineering, pages 87-98. ACM, 2016. 18

[93] Yonghui Wu, Mike Schuster, Zhifeng Chen, Quoc V Le, Mohammad Norouzi, Wolfgang Macherey, Maxim Krikun, Yuan Cao, Qin Gao, Klaus Macherey, et al. Google's neural machine translation system: Bridging the gap between human and machine translation. arXiv preprint arXiv:1609.08144, 2016. 11

[94] Yijun Xiao and Kyunghyun Cho. Efficient character-level document classification by combining convolution and recurrent layers. arXiv preprint arXiv:1602.00367, 2016. 31 
[95] Zhilin Yang, William W Cohen, and Ruslan Salakhutdinov. Revisiting semi-supervised learning with graph embeddings. In Proceedings of the 33rd International Conference on International Conference on Machine Learning-Volume 48, pages 40-48. JMLR.org, 2016. 12

[96] Jonathan S Yedidia, William T Freeman, and Yair Weiss. Understanding belief propagation and its generalizations. Exploring artificial intelligence in the new millennium, 8:236-239, 2003. 21 


\section{Glossary}

abstract syntax tree (AST) A syntax tree which only tracks variable lexemes, such as names or value literals. Non-variable lexical tokens, such as keywords or parentheses, are encoded by the tree's construction.

average precision (AP) The mean precision of a classifier over all classification thresholds. 63

basic block A sequence of instructions. Execution of the block must begin at the head and may only transfer control at the tail. 5

Common Weakness Enumeration (CWE) A set of categories for classifying common defects. See MITRE [57]. 38

control-flow graph (CFG) A directed graph representing the possible paths of execution through a program. Vertices usually represent basic blocks rather than single instructions.

data-flow graph (DFG) A directed graph representing the producers and consumers of values within a program.

dead code Code unreachable from the program's entry point.

domain specific language (DSL) A programming language specialized for a specific domain or task.

fully-connected layer (FC layer) A ubiquitous neural network layer consisting of a learned projection and bias. i.e. $\mathrm{FC}(\mathbf{x})=\mathbf{W} \mathbf{x}+\mathbf{b}$. Typically used in conjunction with a non-linearity.

Gated Recurrent Unit (GRU) A neural model designed for processing sequences. See section 2.3, and Cho et al. [19]. 
Gram matrix A Gram matrix is a positive semidefinite matrix $M \in \mathbb{R}^{|X| \times|X|}$ where $M_{i j}=\left\langle X_{i} \cdot X_{j}\right\rangle$, for the set of vectors $X$. A kernel matrix is a gram matrix where the inner products are computed by a kernel function $k: X \times X \rightarrow \mathbb{R}$. In practice, kernel matrices are not expected to be perfectly positive semidefinite [18, 86]. 15

induced subgraph Given $A=\left(V_{A}, E_{A}\right), B=\left(V_{B}, E_{B}\right), A \subset B$ :

$A$ is an induced subgraph $\Longleftrightarrow \forall x \in V_{A}, y \in V_{A} .(x, y) \in E_{B} \rightarrow(x, y) \in E_{A}$

intermediate representation (IR) A data structure modelling some aspect(s) of a program.

interprocedural analysis (IPA) An interprocedural analysis considers the program being analysed as a whole, rather than multiple disjoint subsets. Non-IPA analyses limit their scope to a single function at most.

Java virtual machine (JVM) A virtual machine created as a compiler target for Java, though other languages now target it as well. (e.g. Clojure, Kotlin, and Scala.)

lexeme A sequence of characters satisfying the pattern definition for a token. Often conflated with the token. 17

Long Short-Term Memory (LSTM) A neural model designed for processing sequences. See section 2.3, and Hochreiter and Schmidhuber [41].

minified source code Source code where all unnecessary whitespace (including comments) has been removed, and identifiers replaced with the shortest possible distinct names. This is often done for compression or cursory obfuscation. 19

multigraph A graph where multiple edges between two vertices is permitted. 14, 23, 24

multi-layer perceptron (MLP) A feed-forward network with at least one hidden layer. See section 2.3. 
neighbourhood (graph) The (open) neighbourhood of a vertex $v, N(v)$, is the set of all incident edges and adjacent vertices; the closed neighbourhood also includes $v$ itself. The $k$-neighbourhood of a vertex $v, N^{k}(v)$, is the subgraph defined by all vertices and edges within radius $k$ from $v$. i. e. the 1-neighbourhood is equivalent to the closed neighbourhood. Note that directed edges are handled as if they were undirected. 22

out-of-vocabulary (OOV) Not present within a model's vocabulary. May also refer to a special symbol used as a substitute for OOV symbols.

partial subgraph A non-induced subgraph.

pure function A side-effect-free subroutine which always produces the same output given the same arguments. 24

receiver operating characteristics (ROC) A 2d plot comparing a classifier's true positive rate against its false positive rate. 56

right-hand-side expression (RHS) The value expression for an assignment operation. In many languages the assignment operator is an infix operator with the value expression appearing on the right and the destination on the left.

single static assignment (SSA) An IR where all variables have exactly one assignment. It is constructed by introducing fresh variables for every assignment and adding $\phi$-nodes to select the correct SSA-variable depending on the executed control flow.

Structured Query Language (SQL) A domain specific language designed for querying databases.

subgraph Given graphs $A=\left(V_{A}, E_{A}\right), B=\left(V_{B}, E_{B}\right)$ :

$A \subset B \Longleftrightarrow V_{A} \subset V_{B} \wedge E_{A} \subset E_{B}$

token A symbolic value representing a basic lexical unit. A token matches a specific pattern of characters (its lexeme), which is tracked for later processing. 
e.g. A NUMERIC_LITERAL token could match multiple notations, such as 5, -0.22 , or 5e-2. 17

type A set of term-level values. e.g. In Java, the type int consists of the integers in the interval $\left[-2^{31}, 2^{31}-1\right]$. $6,28,29$

Unicode Text Format 8-bits (UTF-8) A multi-byte encoding scheme for Unicode codepoints. It is backwards compatible with 7-bit ASCII.

uninhabited type A type consisting of an empty set of values. e.g. void in C-like languages.

void (type) An uninhabited type denoting the return type for subroutines which return no results. Functional languages often use the zero-tuple instead of Void. 29 